Aus der Abteilung Unfallchirurgie, Plastische und Wiederherstellungschirurgie

(Prof. Dr. med. K. M. Stürmer)

im Zentrum Chirurgie

der Medizinischen Fakultät der Universität Göttingen

\title{
Der virtuelle Patient als neue Möglichkeit eines praxisorientierten Medizinstudiums
}

virtusMED: neue Wege in der Sonographieausbildung

\author{
INAUGURAL - DISSERTATION \\ zur Erlangung des Doktorgrades \\ der Medizinischen Fakultät \\ der Georg-August-Universität zu Göttingen
}

vorgelegt von

Wiebke Lena Dittmer

aus

Hamburg

Göttingen 2008 
D e k a n: Prof. Dr. med. C. Frömmel

I. Berichterstatter: Prof. Dr. med. K. Dresing

II. Berichterstatterlin:

III. Berichterstatter/in:

Tag der mündlichen Prüfung: 
Aus Gründen der besseren Lesbarkeit beschränkt sich diese Arbeit auf die Verwendung männlicher Substantive (Student, Dozent, Versuchsleiter), bzw. Pronomina, bezieht sich damit jedoch grundsätzlich im gleichen Maße auch auf weibliche Personen (Studentin, Dozentin, Versuchsleiterin). 


\section{INHALTSVERZEICHNIS}

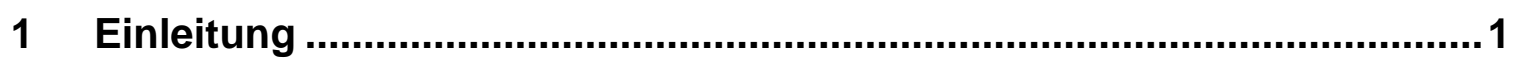

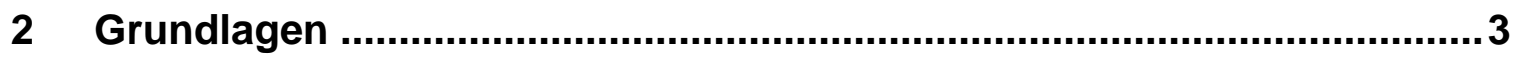

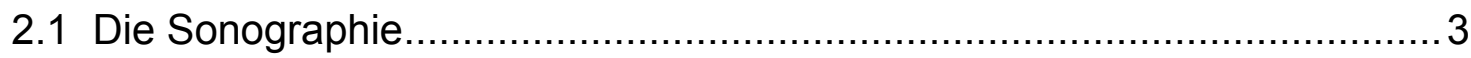

2.1.1 Physikalische Grundlagen des Ultraschalls .................................. 3

2.1.2 Ultraschallerzeugung und Bildsynthese ..................................... 4

2.2 Medizinische Einsatzgebiete des Ultraschalls .....................................

2.3 Der praktische Aspekt der medizinischen Ausbildung ............................. 7

2.3.1 Multimedia in der Medizin - Lernen heute ................................... 8

2.3.2 Ultraschall in der medizinischen Ausbildung ................................. 9

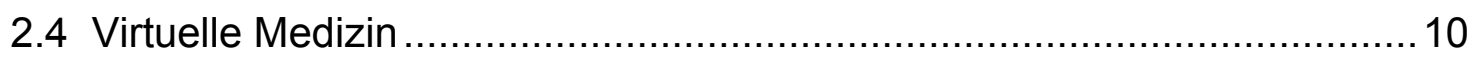

2.4.1 E-Learning (englisch electronic learning - elektronisches Lernen) .. 10

2.4.2 Augmented Reality (englisch augmented reality - erweiterte

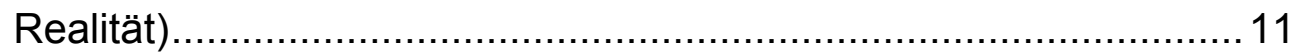

2.4.3 Mock-up (englisch mock-up - Atrappe)...................................... 11

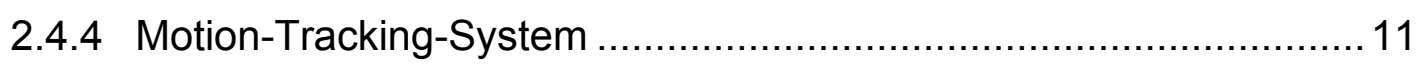

2.4.5 Visible Human Projekt.......................................................... 12

2.4.6 virtusMED (Virtual Scenes for Medical Education and

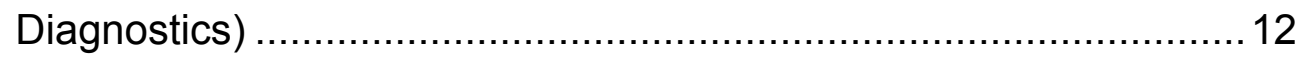

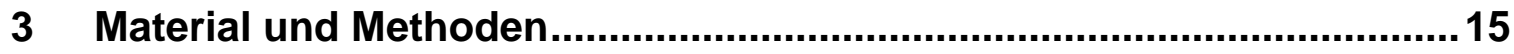

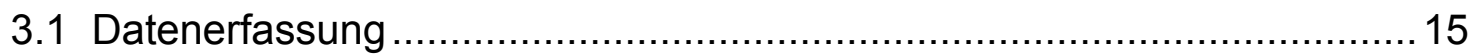

3.2 Stichprobenbeschreibung - Rekrutierung der Studenten........................ 15

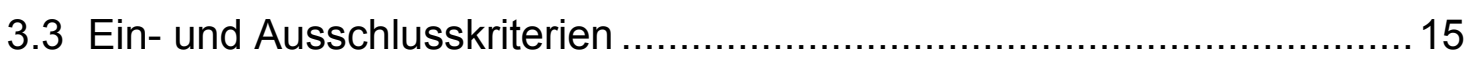

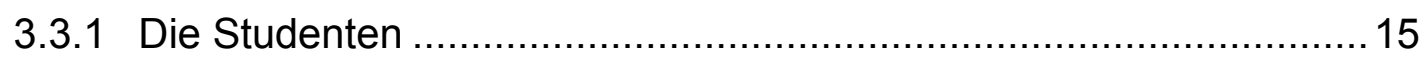

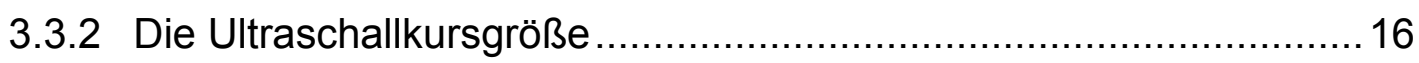

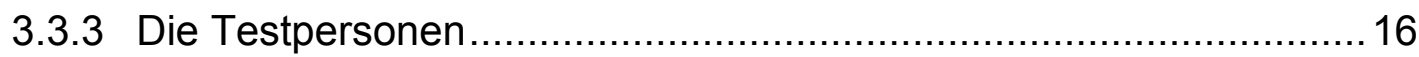

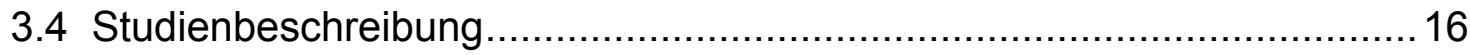

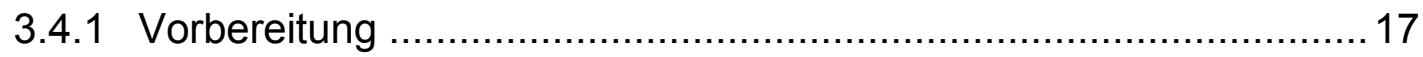

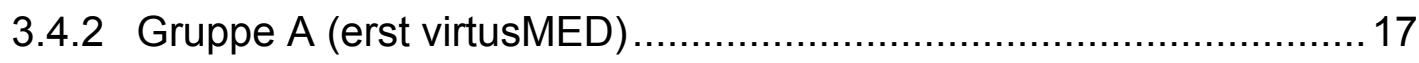

3.4.2.1 Einführung des virtusMED-Systems .................................... 17

3.4.2.2 Selbständiges Explorieren des virtusMED-Systems virtuelle Sonographie ........................................................ 19 
3.4.2.3 Kurztest am virtusMED-System 19

3.4.2.4 Ultraschalluntersuchung einer Testperson - reale Sonographie

3.4.2.5 Einführung des Ultraschallgerätes (SONOLINE Elegra von Siemens)

3.4.2.6 Selbständiges Explorieren am Ultraschallgerät.................... 22

3.4.2.7 Kurztest am Ultraschallgerät....................................... 23

3.4.3 Gruppe B (erst Ultraschall) .............................................. 26

3.4.3.1 Einführung des Ultraschallgerätes (SONOLINE Elegra von Siemens)

3.4.3.2 Selbständiges Explorieren am Ultraschallgerät - reale Sonographie ........................................................... 26

3.4.3.3 Kurztest am Ultraschallgerät ..................................... 26

3.4.3.4 Einführung des virtusMED-Systems.................................26

3.4.3.5 Selbständiges Explorieren des virtusMED-Systems virtuelle Sonographie ..................................................... 26

3.4.3.6 Kurztest am virtusMED-System .................................. 27

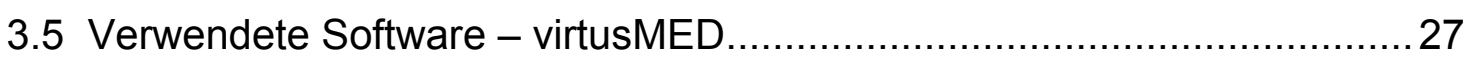

3.5.1 Bedienung des virtusMED-Systems .................................... 29

3.5.1.1 Programmstart ........................................................ 29

3.5.1.2 Die virtuelle Untersuchung ......................................... 31

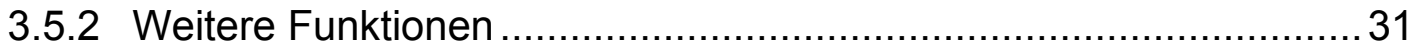

3.5.2.1 Der virtuelle Patient (3D-Ansicht) ................................. 31

3.5.2.2 Organe hinzufügen ............................................... 32

3.5.2.3 Organe benennen ................................................ 32

3.5.2.4 Freeze- und Messfunktion.......................................... 33

3.5.2.5 Tiefeneinstellungen ................................................ 34

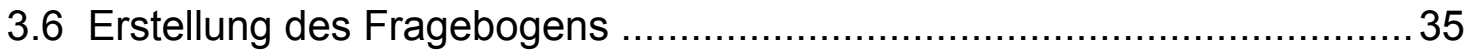

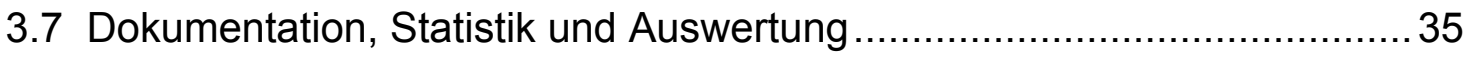

3.7.1 Referenzwert / Goldstandard ................................................ 36

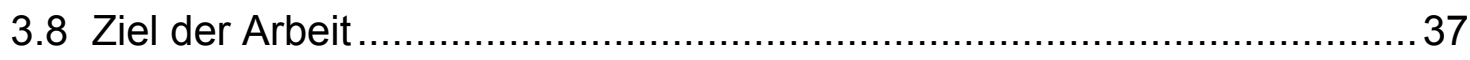

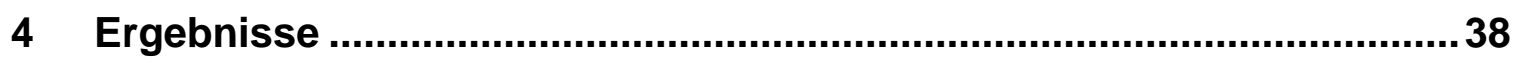

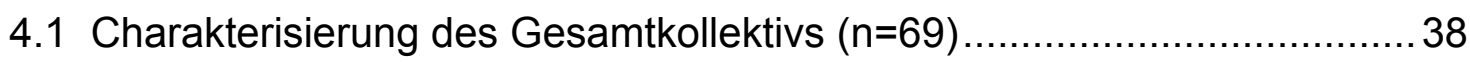

4.1.1 Alter, Semesteranzahl und Geschlechterverteilung ...................... 38 
4.1.2 Erfahrungen am PC 39

4.1.3 Erfahrungen im Bereich der Sonographie 39

4.2 Charakterisierung des getesteten Kollektivs $(n=69)$. 40

4.2.1 Virtuelle Sonographie mit dem virtusMED-System. 40

4.2.2 Reale Sonographie mit dem Ultraschallgerät SOLINE Elegra von Siemens - Gruppe A (erst virtusMED) vs. Gruppe B (erst Sono) ....42

4.2.3 Genauigkeit der Messergebnisse in Abhängigkeit von der Zeit 47

4.2.4 Genauigkeit der Messergebnisse in Abhängigkeit von den Sonographievorkenntnissen.

4.2.5 Genauigkeit der Messergebnisse in Abhängigkeit vom Geschlecht. 48

4.2.6 Genauigkeit der Messergebnisse in Abhängigkeit von der Semesteranzahl .............................................................. 48

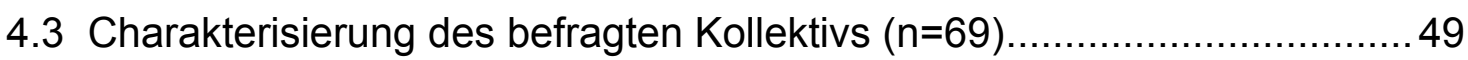

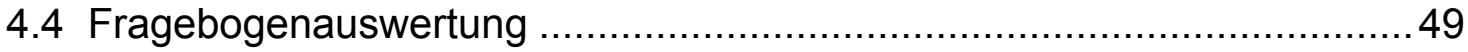

4.4.1 Multiple-choice-Fragen zur Evaluation des virtusMED-Systems in der sonographischen Lehre sowie zum Gebrauch des virtusMEDSystems

4.4.2 Offene Fragen zur Evaluation des virtusMED-Systems in der sonographischen Lehre sowie zum Gebrauch des virtusMEDSystems 51

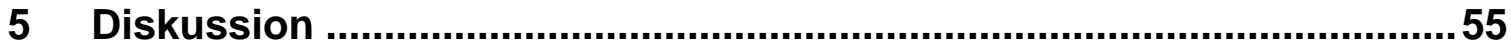

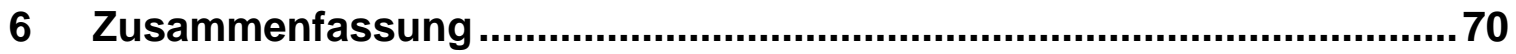

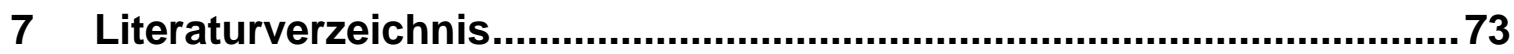

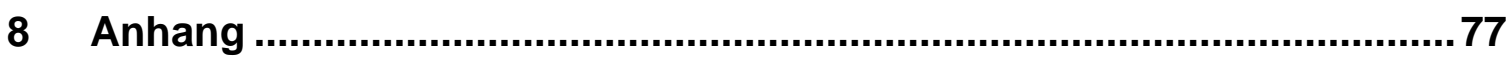

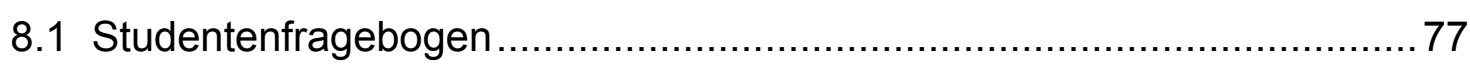

8.1.1 Fragebogen Evaluation virtusMED in der sonographischen Lehre .. 77

8.1.2 Fragen zum Gebrauch des virtusMED-Systems .......................... 79

8.2 Komplette Antworten der offenen Fragen zum Fragebogen Evaluation virtusMED in der sonographischen Lehre ....................................... 80

8.2.1 Komplette Antworten der offenen Fragen zu Fragen zum Gebrauch des virtusMED-Systems ..................................... 82

8.3 Messergebnisse reale Sonographie ............................................ 84

8.3.1 Gruppe A (erst virtusMED) Testperson ${ }_{1}$ und Testperson $2 \ldots \ldots \ldots \ldots \ldots . . . . .84$

8.3.2 Gruppe B (erst Sono) Testperson 1 und Testperson $2 \ldots \ldots \ldots \ldots \ldots \ldots \ldots . . . . . . .66$ 
8.3.3 Versuchsleiter (Goldstandard) Testperson ${ }_{1}$ und Testperson 2 87

8.4 Messergebnisse virtuelle Sonographie. 88

8.4.1 Gruppe A (erst virtusMED) Testperson ${ }_{1}$ und Testperson Th $_{2}$ und Gruppe B (erst Sono) Testperson 1 und Testperson ${ }_{2} \ldots \ldots \ldots \ldots \ldots \ldots \ldots 8$

8.4.2 Versuchsleiter / Referenzwert ............................................. 90 


\section{Einleitung}

Die Sonographie ist nach Angaben der DEGUM (Deutsche Gesellschaft für Ultraschall in der Medizin) das am häufigsten eingesetzte bildgebende Verfahren in der Medizin. Durch stetigen Fortschritt hält es in immer mehr medizinische Fachbereiche Einzug. Im Gegensatz zu Röntgenstrahlen ist Ultraschall für den Patienten unbedenklich, kann beliebig oft wiederholt werden und schafft sofort Resultate. Das nicht-invasive Verfahren ist kostengünstig und ermöglicht durch eine immer besser werdende Bildqualität bewegte Aufnahmen des menschlichen Inneren. Durch kleinere, sogar tragbare Apparate ist Ultraschall mittlerweile fast überall verfügbar. Im Gegensatz zum CT und MRT bieten Ultraschallaufnahmen auf den ersten Blick einen geringeren Informationsgehalt. Dies liegt daran, dass ein physikalisches Grundverständnis zur Interpretation bestimmter Befunde herangezogen werden muss. Auch Erfahrung, ein dreidimensionales Vorstellungsvermögen und handwerkliches Geschick des Untersuchers sind vonnöten, um ein aussagekräftiges Ultraschallbild zu erzeugen. Besonders diese Eigenschaften fehlen dem unerfahrenen Studenten bzw. bereiten ihm Schwierigkeiten bei der Deutung und Erzeugung der zweidimensionalen, graustufigen Bilder.

Das Medizinstudium soll nicht nur theoretisches Faktenwissen vermitteln, sondern auch praktische Kenntnisse z.B. zur Diagnostik (Neue AO 2002). Die Praxis kommt gegenüber der Theorie jedoch oft zu kurz und aktives Lernen tritt hinter dem passiven Wissenserwerb in den Hintergrund (Heimpel 1998). Ein Grund dafür ist unter anderem eine immer größere Anzahl von Studierenden, die immer kürzere Verweildauer von Patienten und das Nicht-Vorhandensein des „richtigen“ Patienten für den Unterricht am Krankenbett.

Neben den „klassischen“ Lernformen - Lehrbuch, Vorlesung und Unterricht am Krankenbett - kommen in der medizinischen Ausbildung zunehmend multimediale Produkte zum Einsatz (Krüger-Brand 1999, 2002). Diese Produkte ermöglichen dem Studenten mittels Selbststudium in individuellem Lerntempo (Heimpel 1998) zeit- und ortsunabhängig (Krüger-Brand 2002) Wissen zu erwerben, zu vertiefen oder zu wiederholen. Mit Hilfe von Simulationen können komplexe Abläufe oder topographisch-funktionelle Gegebenheiten einfach 
visualisiert werden (Floto 2002). Der virtuelle Patient hält somit zunehmend Einzug in das Medizinstudium. Der Vorteil: ein virtueller Patient kann beliebig oft und ohne sich zu beschweren untersucht werden (Page 2002). Eine Alternative zu den Theorie abprüfenden Multiple-choice-Fragen sind praktisch-klinische Prüfungen (OSCE - Oral Standardized Clinical Examination). Diese standardisierten Tests werden zum Teil durch Probanden, aber auch Simulatoren realisiert. Verschiedene Simulatoren bieten Studenten ein praxisnahes und handlungsorientiertes Studium.

Da die sonographische Ausbildung während des Studiums oft zu kurz kommt (Hoffmann 2003), ist das Ziel vorliegender Arbeit zu prüfen, ob der Einsatz eines Sonographie-Simulators hierbei Abhilfe schaffen kann. Es soll untersucht werden, ob das virtusMED-System einen Beitrag zur Verbesserung eines praxisorientierten Medizinstudiums vor allem in der Sonographieausbildung leistet und ob Studenten bessere Messergebnisse bei der Sonographie durch ein virtuelles Vortraining mit Hilfe dieses Systems erzielen. 


\section{Grundlagen}

\subsection{Die Sonographie}

Seit über 50 Jahren findet die Sonographie Anwendung in der medizinischen Diagnostik. Elektrotechnik und Computerentwicklung ermöglichen eine immer bessere Bildqualität und damit eine höhere diagnostische Aussagekraft. Der Einsatz ist kostengünstig, beliebig oft wiederholbar, schafft sofort Resultate und ist dabei unbedenklich für den Patienten. Es verwundert deswegen nicht, dass die Sonographie das am häufigsten zu diagnostischen Zwecken eingesetzte bildgebende Verfahren in der Medizin ist.

Die Kenntnis der physikalischen und technischen Grundlagen ist Voraussetzung für die Interpretation und Anfertigung sonographischer Bilder.

\subsubsection{Physikalische Grundlagen des Ultraschalls}

Ultraschallwellen sind mechanische Schwingungen, die in ihrer Ausbreitung im Gegensatz zu elektromagnetischen Schwingungen wie Licht und Röntgenstrahlen an Materie gebunden sind (vgl. Kratochwil 1977). Da biologisches Gewebe physikalisch als zähe Flüssigkeit aufgefasst werden kann, breiten sich Ultraschallwellen ausschließlich in Form von Longitudinalwellen aus. Im Gegensatz zu Transversalwellen sind Schwingungs- und Ausbreitungsrichtung bei Longitudinalwellen identisch. Je fester und unelastischer das Gewebe, desto besser und schneller breitet sich die Schallwelle aus. Die Schallgeschwindigkeit ist somit eine gewebe- und materialspezifische Größe und ist durch die Dichte und Kompressibilität des Gewebes charakterisiert. Im menschlichen Körper beträgt die Schallgeschwindigkeit $1490 \mathrm{~m} / \mathrm{s}$ bis $1660 \mathrm{~m} / \mathrm{s}$. Nur im Knochen liegt die Schallgeschwindigkeit aufgrund der wesentlich höheren Dichte und des höheren Elastizitätsmoduls bei 3360 m/s (vgl. Kratochwil 1977).

Der Frequenzbereich der Ultraschallwellen, die in der medizinischen Diagnostik Anwendung finden, liegt zwischen ca. $1 \mathrm{MHz}$ (1 Megahertz; 1 Million Schwingungen pro Sekunde) und $20 \mathrm{MHz}$.

Die Beziehung von Schallgeschwindigkeit $c$, Wellenlänge $\lambda$ und Frequenz $f$ wird in der Wellengleichung dargestellt: $\lambda=\mathrm{c} / \mathrm{f}$. 
Die Wellenlänge liegt für die Sonographie in der Größenordnung $0,1 \mathrm{~mm}$ bis 1 $\mathrm{mm}$ und gilt als grobes Maß für die Detailerkennbarkeit. Kleinere Strukturen können nicht einzeln abgebildet werden (vgl. Delorme und Debus 2005). Mit steigender Frequenz nimmt das Auflösungsvermögen zu und die Eindringtiefe ab, was dazu führt, dass tiefere Strukturen schlechter erkennbar sind.

Beim Durchtritt durch Gewebe wird Ultraschall durch Reflexion und Brechung, Streuung und Absorption geschwächt. Art und Ausmaß der Schwächung hängen vom durchlaufenen Gewebe ab. Es ist wichtig zu wissen, dass das Ultraschallbild keine direkte anatomische Abbildung ist, sondern eine Überlagerung sehr vieler Ultraschallwellen, die dem Gewebe ein typisches Aussehen verleihen. Man spricht in diesem Zusammenhang von Textur oder Echostruktur. Ein differenziertes Ultraschallbild kann nur entstehen, wenn die Gewebe eine unterschiedliche akustische Impedanz (Schallwellenwiderstand oder Schallleitfähigkeit) besitzen, da die Gewebe nur so voneinander unterschieden werden können (vgl. Delorme und Debus 2005).

\subsubsection{Ultraschallerzeugung und Bildsynthese}

Zur Erzeugung von Ultraschall wird der 1880 von den Geschwistern Curie entdeckte piezoelektrische Effekt genutzt. Diese beobachteten, dass sich eine elektrische Spannung erzeugen lässt, wenn man bestimmte Kristalle (z.B. Quarz und Turmalin) deformiert. Die entstehende Ladung ist dabei direkt proportional der mechanischen Verformung. Das heißt, je höher der auf den Kristall einwirkende Druck, desto größer die Spannung. Die Vorzeichen der Ladung ändern sich abhängig davon, ob der Kristall gestaucht oder gestreckt wird. Wird nun in umgekehrter Weise eine elektrische Spannung an einen Piezokristall angelegt, verlängert oder verkürzt sich dieser je nach Polung der Spannung. Die induzierte Verlängerung oder Verkürzung ist direkt proportional der angelegten Spannung. Durch Anlegen einer Wechselspannung werden elektrische Schwingungen in mechanische umgewandelt - der Kristall beginnt zu schwingen. Sinterkeramiken oder mit kristallinen Anteilen besetzte Kunststoffe (z.B. PVDF - Polyvinylidene Fluoride) werden zur Herstellung moderner Ultraschallsonden gebraucht (vgl. Kratochwil 1977 und Frey et al. 2006). 
Die in der medizinischen Diagnostik eingesetzte Ultraschalltechnik basiert auf dem Echo-Impuls-Verfahren, das unter anderem auch von Fledermäusen z.B. zur Orientierung angewandt wird. In diesem Fall wird der Piezokristall sowohl als Sender als auch Empfänger von Ultraschallwellen verwendet. Nach Aussenden eines kurzen Schallimpulses kann so über den Zeitverzug des Echos (der zurücklaufende Teil des Schallimpulses) die Entfernung des reflektierten Gewebes ermittelt werden $(z=c \cdot t$, Betrag durch Faktor 2 dividieren; $z=$ Wegstrecke, $c=$ Ultraschallgeschwindigkeit, $t=$ Zeitdifferenz). Außerdem können aus der Stärke des Echos Rückschlüsse auf die Impedanz des reflektierten Objektes gezogen werden (vgl. Delorme und Debus 2005).

\subsection{Medizinische Einsatzgebiete des Ultraschalls}

Ultraschall ist das am häufigsten eingesetzte bildgebende Verfahren in der Medizin. Seit mehr als 10 Jahren stellt es mit einem Anteil von 52\% das von Vertragsärzten am meisten abgerechnete bildgebende Verfahren in der Bundesrepublik dar. Schätzungen zufolge wird der Anteil auch in den nächsten Jahren, besonders im ambulanten Bereich kontinuierlich steigen (Kratzer et al. 2000).

$\mathrm{Ob}$ als stationäres Gerät, um eine Abdomenübersicht zu erhalten, als handliches Gerät im Notarztwagen, um Herzinfarkt oder Schlaganfälle festzustellen (DEGUM Presseinfo Mai 2005), um Schmerzmedikamente gezielt an Nervenbahnen zu spritzen (DEGUM Presseinfo Juni 2007) oder eine Lungenembolie direkt am Krankenbett zu diagnostizieren (DEGUM Presseinfo Mai 2006), durch den kontinuierlichen technischen Fortschritt hält der Ultraschall in immer mehr medizinische Fachbereiche Einzug. Als nicht-invasives Verfahren ist Ultraschall vielerorts unbedenklich einsetzbar, aber auch intraoperativ findet er Anwendung.

Blutströmungen lassen sich bequem mit der Farbduplexsonographie untersuchen. In der Gynäkologie sowie Kardiologie kommen 3D- oder 4DSonographiegeräte zum Einsatz, um Missbildungen während der Schwangerschaft frühzeitig zu erkennen bzw. Herzklappenerkrankungen aufzudecken.

Es erscheint unglaublich, wenn man bedenkt, was moderne Ultraschallgeräte zu leisten im Stande sind. Sie können nicht nur zur Diagnostik bereits bestehender 
Erkrankungen eingesetzt werden, sondern auch zur Früherkennung oder Prävention. Mittels einer transkraniellen Sonographie (TCS) können beispielsweise schon im Frühstadium des Morbus Parkinson „hyperechogene“ Zonen in der Substantia nigra dargestellt werden, die im CT und MRT sogar im Spätstadium unsichtbar bleiben (DEGUM Presseinfo Juli 2006). Auch bei der Früherkennung von Lebertumoren ist Ultraschall ein wichtiges diagnostisches Verfahren. Tumoren lassen sich bereits ohne Kontrastmittel im Ultraschall identifizieren. Der Einsatz von Kontrastmittel steigert die Sensitivität jedoch deutlich und kann die Aussagekraft eines CTs sogar überschreiten. Auf ein aufwendiges und strahlenbelastendes Computertomogramm könnte in diesem Fall verzichtet werden (DEGUM Presseinfo September 2004). Moderne Ultraschallgeräte haben eine immer höhere Auflösung und ermöglichen es, feinste Strukturen wie Nervenbahnen und Bindegewebe sehr genau darzustellen. Noch bevor ein Patient Schmerzen verspürt, ist es mittels Ultraschall möglich, degenerative Veränderungen beispielsweise durch Überlastung an Sehnen, Muskeln und Bändern zu diagnostizieren. „Entzündungsprozesse an Achillessehen, Schleimbeutel oder umgebendem Gewebe werden auf dem Ultraschallbild, nicht aber auf dem Röntgenbild sichtbar“, sagt Dr. Sattler von der DEGUM (Deutsche Gesellschaft für Ultraschall in der Medizin). Ultraschall ist in der Gelenkdiagnostik von Sportverletzungen folglich sogar präventiv einsetzbar (DEGUM Presseinfo Mai 2005).

Aufgrund der Kombination von mechanischer Vibrationswirkung und thermischer Wirkung mit Vasodilatation findet Ultraschall auch in der Physiotherapie Anwendung. Er wirkt außerdem analgesierend, permeablitätssteigernd, hyperämisierend und muskelrelaxierend.

Variable Größen und verschieden geformte Ultraschallsonden machen die Untersuchung unterschiedlicher Organe möglich. So können beispielsweise Strukturveränderungen im Ösophagus, Magen oder Darm durch eine Endosonographie untersucht werden, Ovarien und Uterus können mittels vaginaler Sonographie beurteilt werden und die transösophageale Echographie ist bei der Suche nach Thromben im Vorhof oder Herzohr behilflich. Viele Patienten lassen sich hervorragend mittels Ultraschall untersuchen. Probleme können jedoch stark übergewichtige Patienten bereiten, da die Schallwellen 
durch die dicke Binde- oder Fettgewebsschicht abgeschwächt werden. Auch luftgefüllte Darmschlingen sind bei einer Abdomensonographie kontraproduktiv.

\subsection{Der praktische Aspekt der medizinischen Ausbildung}

Nach Angaben der Neuen Approbationsordnung (Neue AO 2002) ist das Ziel der ärztlichen Ausbildung der wissenschaftlich und praktisch in der Medizin ausgebildete Arzt, der zur eigenverantwortlichen und selbständigen ärztlichen Berufsausübung, zur Weiterbildung und zu ständiger Fortbildung befähigt ist. Dabei sollte die Ausbildung zum Arzt auf wissenschaftlicher Grundlage und praxis- und patientenbezogen durchgeführt werden. Es wird unter anderem großer Wert darauf gelegt, die für das ärztliche Handeln erforderlichen allgemeinen Kenntnisse, Fähigkeiten und Fertigkeiten in Diagnostik, Therapie, Gesundheitsförderung, Prävention und Rehabilitation auf der Basis des aktuellen Forschungsstandes zu vermitteln (Neue AO 2002).

Nach wie vor wird der überwiegende Teil des medizinischen Wissens theoretisch über Bücher erarbeitet oder durch Vorlesungen vermittelt. Es handelt sich bei dieser Art Wissensvermittlung um passives Lernen. Viele Vorlesungen werden durch Powerpointpräsentationen mit sehr großem Informationsgehalt und oft einer zu schnellen Bildabfolge ergänzt. Studenten fällt es teilweise schwer zu folgen und der Wissenszugewinn ist gering. Eine Interaktion zwischen Student und Dozent findet hier nicht statt. Seminare ermöglichen an dieser Stelle einen Ausgleich und bilden ein Diskussionsforum für Studenten und Dozenten. Anhand von Fallbeispielen ist es möglich, in einer solchen Runde theoretisch erlerntes Wissen praxisorientiert anzuwenden. Neben den theorieabfragenden Multiplechoice-Fragen kommen mittlerweile vermehrt praktisch-klinische Prüfungen, sog. OSCEs (Oral Standardized Clinical Examinations), zum Einsatz.

Der Unterricht am Krankenbett ist eine weitere Alternative, um Studenten den Praxisbezug der erlernten Theorie zu vermitteln. Ein Problem dabei ist die Patientenverfügbarkeit. Es gibt immer mehr Studierende für eine gleich bleibende Anzahl von Patienten, deren Verweildauer in der Klinik immer kürzer wird. Hinzukommt, dass oft nicht der „richtige“ Patient mit zuvor erlerntem Krankheitsbild zur Verfügung steht und der Student von der Geduld des Patienten abhängt. Das Learning-by-doing ist aus diesem Grund nicht immer möglich und kommt oft zu kurz. 
Zukünftig könnten virtuelle Patienten oder neuartige computerbasierte Simulationen einen alternativen Beitrag für ein praxisorientiertes Studium leisten. Durch ihren Anwendungsbezug eröffnen diese Programme die Möglichkeit, Faktenwissen in medizinisches Handlungswissen zu überführen (vgl. Rosendahl 2003).

\subsubsection{Multimedia in der Medizin - Lernen heute}

Die „klassischen“ Lernformen - Vorlesung, Lehrbuch und Lernen am Krankenbett - werden zunehmend durch so genanntes E-Learning (elektronisches Lernen) mittels computergestützter oder webbasierter Lernsoftware ergänzt (Krüger-Brand 2002).

Nach Angaben von Prof. Dr. med. J. Bernauer, Arzt und Informatiker an der Fachhochschule Ulm, wird künftig interaktiv, adaptiv, jederzeit und überall gelernt. Tatsächlich ist es dem Studenten möglich, zeit- und oft sogar ortsunabhängig (bei webbasierten Lernsystemen) mittels Selbststudium Zugang zu Lerninhalten durch computergestützte Lernsysteme zu bekommen (KrügerBrand 2002).

Nitzsche behauptet, dass die Medizin ein ausgesprochen sinnliches und daher multimediales Fach ist (Nitzsche 2002). Viele Lerninhalte des Medizinstudiums lassen sich daher mit multimedialen Produkten einfacher und effektiver vermitteln als mit traditionellen Lehrmaterialien (Hirsch 1999). Einem Student wird es vermutlich leichter fallen, beispielsweise zu verstehen, wie sich ein Bild bei der Sonographie durch Kippen des Schallkopfs verändert, wenn er dies selber, sei es mittels eines Simulationsprogramms oder des originalen Schallgerätes, ausprobiert, als wenn es ihm theoretisch erklärt wird. Der Einsatz von Multimediaprodukten vermittelt oft einfacher ein tieferes Verständnis für den Sachverhalt und bietet damit eine sinnvolle Ergänzung zu einer praxisorientierten Ausbildung (Nitzsche 2002).

Besondere Impulse für die Entwicklung weiterer Wissensmedien gehen von den auf Bildgebung und Morphologie basierten Fachgebieten, wie beispielsweise der Anatomie, der Radiologie oder operativen klinischen Fächern aus, in denen topographische Lagebeziehungen eine wichtige Rolle spielen (Floto 2002). Ein Beispiel für ein derartiges Programm ist das virtusMED-System, das die 
interaktive Exploration volumetrischer Bilddaten, z.B. einer zusammengehörigen Menge transversaler CT-Schnittaufnahmen mittels Untersuchungssonde, ermöglicht. Besonderes Augenmerk liegt bei dieser Lernsoftware auf der Mensch-Maschine-Schnittstelle, d.h. auf der Interaktion des Anwenders mit der generierten virtuellen Szene (Teistler 2004). Der virtuelle Patient wird den realen Patient und den Unterricht am Krankenbett mit Sicherheit nicht ersetzen können, bietet aber hinsichtlich der praxisorientierten Ausbildung eine Ergänzung sowie die Möglichkeit, Wissen und Fertigkeiten zu üben, zu vertiefen und aktiv anzuwenden.

\subsubsection{Ultraschall in der medizinischen Ausbildung}

Nach der Neuen Approbationsordnung sollten Studenten unter anderem allgemeine Fähigkeiten und Fertigkeiten in Diagnostik vermittelt bekommen (Neue AO 2002). Welche diagnostischen Verfahren dazugehören, ist jedoch nicht klar definiert. Eine strukturierte sonographische Ausbildung ist beispielsweise weder im Medizinstudium noch in der Facharztausbildung vorgesehen.

Die Sonographie ist das am häufigsten eingesetzte bildgebende Verfahren in der Medizin und findet in vielen Fachbereichen Anwendung. Aus diesem Grund scheint es sinnvoll, Studenten bereits während des Studiums Theorie und Praxis dieser Technik näher zu bringen.

Studenten ist oft unerklärlich, was sich hinter den verschiedenen Grautönen der bewegten Bilder verbirgt. Unumstritten ist, dass die Wertigkeit der Befunde bei der Ultraschalluntersuchung stark von der Erfahrung des Untersuchers abhängt und die Rate von Fehlbefunden beispielsweise deutlich höher ist als bei CToder MRT-Bildern. Physikalische und technische Grundlagen lassen sich aber ebenso wie manuelle Fertigkeiten durch Übung erwerben und können somit einer Fehlinterpretation vorbeugen.

Um das Defizit der Sonographiekenntnisse auszugleichen, bietet die Deutsche Gesellschaft für Ultraschall in der Medizin (DEGUM) spezielle Fortbildungskurse für verschiedene Fachbereiche der Medizin an. Es wurde eine dreistufige Qualifikation definiert. Eine flächendeckende Versorgung in der Ultraschalldiagnostik in der BRD mit einer qualifizierten Basisdiagnostik und 
einer ausreichenden Dichte von Referenzzentren in Klinik und Praxis ist das Ziel dieses Drei-Stufen-Konzeptes (vgl. http://www.degum.de/567.html).

Die erste der drei Stufen entspricht einer Basisausbildung. Stufe zwei oder drei entspricht einer höheren Qualifikation und wird nach einer Prüfung zuerkannt. Sie garantiert für ein bestimmtes Anwendungsgebiet eine hohe oder sehr hohe Expertise. Um die Qualität zu sichern, muss das Zertifikat alle sechs Jahre neu erworben werden sowie ein qualitativ hochwertiges Ultraschallgerät zur Verfügung stehen.

\subsection{Virtuelle Medizin}

Medizinstudenten sowie angehende Ärzte haben nur wenige Möglichkeiten während ihrer theorielastigen Ausbildung, praktische Erfahrungen am Patienten zu sammeln. Oft müssen sie nach dem Trial-and-error- oder Learning-by-doingVerfahren vorgehen, was nachteilig für den Patienten sein kann (vgl. Page 2002). Computersysteme, Modellierungen und Simulationen bieten eine Alternative. Sie ermöglichen seit einiger Zeit neben der konventionellen Medizin, die sich in praktische und theoretische Medizin teilt, ein Drittes, Neues: Virtuelle Medizin (Pretschner 2001). Der Vorteil der virtuellen Medizin: „ein virtueller Patient beschwert sich nicht" (Page 2002).

\subsubsection{E-Learning (englisch electronic learning - elektronisches Lernen)}

Unter E-Learning werden alle Formen des Lernens verstanden, bei denen digitale Medien für die Präsentation und Verteilung von Lernmaterialien und/oder zur Unterstützung zwischenmenschlicher Kommunikation verwendet werden.

Verschiedene Technologien kommen hierbei zum Einsatz. Rechnergestütztes Lernen, bei dem CD-ROM oder DVD verwendet werden, wird unter dem seit den 80er Jahren bekannten Begriff Computer-Based Training zusammengefasst. Eine Weiterentwicklung mit netzbasiertem Lernangebot bildet das so genannte Web-Based Training (WBT).

Bei den Lernsystemen lassen sich grob folgende, zum Teil auch kombinierte Formen unterscheiden, wobei verwendete Techniken und Methoden sehr verschieden sein können: 
- Präsentationssysteme, bei denen Informationseinheiten hypertextual verbunden sind und multimedial präsentiert werden;

- Tutorielle Systeme, bei denen ein virtueller Tutor den Studierenden in seinen Lernaktivitäten begleitet und beurteilt sowie den Unterricht an den Lernfortschritt anpasst;

- Simulationssysteme, in denen beispielsweise bestimmte Untersuchungstechniken virtuell geübt werden können, oder Probleme und Fälle zu lösen sind (Krüger-Brand 1999).

\subsubsection{Augmented Reality (englisch augmented reality - erweiterte Realität)}

Bei der „virtuellen Realität“ taucht der Benutzer komplett in eine virtuelle Welt ein. Im Gegensatz dazu steht die „erweiterte Realität“ (Augmented Reality), die sowohl reale als auch virtuelle Bestandteile enthält, wobei der reale Teil überwiegt und durch den virtuellen Teil ergänzt wird.

Nach Azuma (1997) sind für die „erweiterte Realität“ drei Merkmale charakteristisch:

- reale und virtuelle Informationen werden kombiniert (teilweise überlagert),

- reale und virtuelle Objekte stehen dreidimensional zueinander in Bezug,

- der Benutzer kann in Echtzeit interagieren.

\subsubsection{Mock-up (englisch mock-up - Atrappe)}

Der Begriff Mock-up bezeichnet eine Atrappe (Modell) und stammt ursprünglich aus der Luftfahrtindustrie. Mittlerweile wird er auch von anderen Bereichen übernommen. Das Mock-up (auch englisch mock-up mode - Lehrmodell oder Übungstest) ist ein reales Teilmodell eines größeren Ganzen, das zu Übungszwecken verwendet wird.

\subsubsection{Motion-Tracking-System}

Zur Positionsbestimmung eines realen Objektes werden sog. Motion-TrackingSysteme (kurz: Trackingsystem) eingesetzt. Sie lassen sich nach ihrer technischen Realisierung und der Anzahl der gemessenen Freiheitsgerade 
(degrees of freedom) klassifizieren. Für Anwendungen im Bereich Virtueller Realität oder Augmented Reality sind Trackingsysteme ein elementarer Baustein (Teistler 2004).

\subsubsection{Visible Human Projekt}

Das Visible Human Projekt (The Visible Human Projekt 2003 a) liefert neben CTund MRT-Aufnahmen digitale photographische Schnittbilder des menschlichen Körpers. Im Jahre 1994 wurden von einem männlichen Leichnam 1.871 photographische Schnittbilder (1 $\mathrm{mm}$ Schichtabstand, 0,33 $\mathrm{mm}$ Pixelgröße) sowie CT- und MRT-Aufnahmen mit unterschiedlichen Schnittabständen der weltweiten Öffentlichkeit zur Verfügung gestellt (Visible Human Male). Von einem weiblichen Leichnam wurden ein Jahr später sogar 5.189 photographische Schnittbilder (0.33 $\mathrm{mm}$ Schichtabstand) angefertigt und veröffentlicht (Visible Human Female) (The Visible Human Projekt 2003 b).

Diese Datensätze werden besonders von Forschergruppen verwendet, die sich mit medizinischer Bildverarbeitung und Visualisierung beschäftigen. Die Schnittbilder werden dabei als "Goldstandard" angesehen und werden als Grundlage zur Erzeugung detaillierter virtueller Körpermodelle verwendet (Dormeier und Teistler 2001).

\subsection{6 virtusMED (Virtual S cenes for Medical Education and Diagnostics)}

Die Transparenz des menschlichen Körpers ist für die Diagnostik und Therapie in der Medizin von enormem Wert. Die Kenntnis über anatomische und physiologische Veränderungen sind nötig, um Krankheiten entdecken und behandeln zu können. Eine wesentliche Rolle spielt dabei deren Visualisierung die medizinische Bildgebung (Dormeier und Teistler 2001).

virtusMED ist eine Lernsoftware, die eine neuartige, interaktive Exploration des menschlichen Körpers in Echtzeit ermöglicht und damit einen Beitrag zur Visualisierung schafft. Die Daten für dieses Programm liefert der Visible Human Male, dessen fotographische Schnittbilder, CT- und MRT-Bilddaten sowohl in zweidimensionale (Ultraschallmodus) als auch in dreidimensionale Szenen umgesetzt werden. 
Der Anwender dieses Programms hat die Möglichkeit, einen virtuellen Patienten zu untersuchen, der vor ihm auf dem Tisch liegt. Als Orientierung dient inm ein Poster oder ein Torso, der mit der Virtualität in Übereinstimmung gebracht wird. Mit einer Untersuchungssonde (Analogie: Ultraschallsonde) können beliebige Körperregionen aufgesucht werden und in Echtzeit fotographische Schnittbilder beliebiger Orientierung erzeugt werden (Teistler 2004). Zwei Monitore stehen dem Untersucher zur Verfügung. Auf dem einen wird ein zweidimensionales Bild generiert (Ultraschallmodus), welches der Schallkopfposition entspricht. Der zweite Bildschirm zeigt eine dreidimensionale Darstellung des virtuellen Patienten, wobei die Untersuchungssonde und der Weg der Schallwellen ebenfalls abgebildet werden. Teistler meint, dass dem unerfahrenen Untersucher durch diese zusätzliche Visualisierung eine bessere räumliche Vorstellung ermöglicht wird.

Entsprechend der Bildgebung in der Sonographie wird durch freie Bewegung der Sonde im Raum ein der jeweiligen Position und Orientierung entsprechendes Schnittbild generiert (Teistler 2004). Für diese Funktion ist die Verwendung einer speziellen Hardware, eines so genannten Trackingsystems, notwendig. Ansonsten ist jedoch keine weitere Hardware oder ein spezieller GrafikHochleistungsrechner vonnöten. Da das System auf der Basis eines StandardPCs realisiert worden ist, ist es zusätzlich als kostengünstig einzustufen (http://www.telemed-atlas.de/?cmd=detail\&id=61\&seite=1\&db=projekte).

Besonderes Augenmerk wurde bei dem virtusMED-System auf die MenschMaschine-Schnittstelle, d.h. auf die Interaktion des Anwenders mit der generierten virtuellen Szene gelegt (Teistler 2004).

Dormeier sagt, dass dieses viel versprechende System hilfreich beim Lernen und Üben praktischer Fähigkeiten ist und besonders dort zum Einsatz kommen kann, wo kein Patient zur Verfügung steht. Dresing vermutet, dass Studenten durch die dreidimensionale Darstellung schneller und besser ein Verständnis für den Körper entwickeln (Rhein-Zeitung online 2002).

Für die Entwicklung des virtusMED-Systems haben Teistler und das Team vom Institut für Medizinische Informatik der Technischen Universität Braunschweig bereits mehrfach Auszeichnungen erhalten, unter anderem 2001 auf dem internationalen Radiologenkongress, RSNA, in Chicago. 
Verwendung könnte dieses neuartige System zukünftig sowohl in der anatomischen als auch in der sonographischen Aus- und Weiterbildung von Medizinstudenten und Ärzten finden.

Ob Studenten sich mit Hilfe dieses Lernprogramms besser bei der Sonographie orientieren können, wie sie das Programm bewerten und ob sie einen zukünftigen Einsatz in der Lehre für sinnvoll erachten, soll vorliegende Studie zeigen. 


\section{Material und Methoden}

\subsection{Datenerfassung}

Die Datenerhebung fand im Rahmen eines zweistündigen KompaktUltraschallkurses statt, der zwei bis viermal pro Woche im Sommersemester 2007 an der Georg-August-Universität Göttingen angeboten wurde.

Durch einen Fragebogen wurden folgende Daten erfasst:

Name, Alter, Geschlecht, Semesteranzahl, Erfahrungen am PC (Textverarbeitung, Internet, Email, Programmierung, eigener PC zu Hause), Sonographievorkenntnisse.

Im Rahmen des Kurses hatten die Studenten verschiedene Messungen durchzuführen, deren Ergebnisse gespeichert wurden.

Ein Ethikantrag war nicht erforderlich, da es sich bei den Teilnehmern nicht um Patienten, sondern um Studierende handelte, die freiwillig an der Studie teilnahmen.

\subsection{Stichprobenbeschreibung - Rekrutierung der Studenten}

Die Studie befasst sich mit einem Gesamtkollektiv von 69 Studenten, die im Sommersemester 2007 an der Georg-August-Universität Göttingen studierten. Mittels Flyer wurde im Kurs „Medizinische Basisfähigkeiten“ (erstes klinisches Semester) für die Teilnahme an der Studie bzw. einem zweistündigen Ultraschallkurs geworben. Interessierte Studenten nahmen freiwillig an der Studie teil.

\subsection{Ein- und Ausschlusskriterien}

\subsubsection{Die Studenten}

Voraussetzung für die Teilnahme an der Studie (einem zweistündigen Ultraschallkurs) waren ausreichende anatomische Kenntnisse. Dies wurde sichergestellt, indem die Studie nur für Studenten des klinischen Studienabschnitts angeboten wurde. Die Studenten sollten keine bzw. möglichst geringe Vorkenntnisse im Umgang mit der Sonographie besitzen. Aus diesem 
Grund wurden bevorzugt Studenten des ersten klinischen Semesters für die Studie ausgewählt.

\subsubsection{Die Ultraschallkursgröße}

Drei Studenten (eine Kleingruppe) konnten gleichzeitig an einem Ultraschallkurs teilnehmen. Die Studenten hatten die Möglichkeit, sich selbständig, unabhängig von Geschlecht und Alter, zusammenzufinden. Eine Kleingruppe wurde anschließend nach dem Zufallsprinzip der Gruppe A (erst virtusMED, dann reale Sonographie) oder Gruppe B (erst reale Sonographie, dann virtusMED) zugeteilt.

\subsubsection{Die Testpersonen}

Zwei Studentinnen (Testperson ${ }_{1}$ und Testperson 2 ) fungierten als „Patient“ für die Studenten. Im Rahmen des Ultraschallkurses hatten die Studenten die Aufgabe, eine der beiden Testpersonen sonographisch zu untersuchen. Es wurde darauf geachtet, dass die Bedingungen für die Studenten möglichst identisch und für Anfänger in der Sonographie geeignet waren. Beide Testpersonen waren zum Zeitpunkt der Studie schlank $\left(\mathrm{BMI}_{1}=19,12, \mathrm{BMI}_{2}=18,13\right)$ und gut $\mathrm{zu}$ untersuchen.

\subsection{Studienbeschreibung}

69 Studenten nahmen an einem zweistündigen Ultraschallkurs teil, um herauszufinden, ob das virtusMED-System einen positiven Lerneffekt auf Studenten in der sonographischen Ausbildung hat. Drei Studenten (eine Kleingruppe) nahmen gleichzeitig an einem Ultraschallkurs teil. Diese Kleingruppen wurden nach dem Zufallsprinzip auf zwei Gruppen (Gruppe A und B) aufgeteilt und anschließend miteinander verglichen. Die eine Gruppe (Gruppe A) erhielt ein Vortraining mit dem virtusMED-System, die andere Gruppe (Gruppe B) wurde direkt an das Ultraschallgerät geführt. 
Nullserie:

Nach Konzipierung der Studie wurde der Studienablauf unter realen Bedingungen getestet.

Für Gruppe A und B wurde geprüft, ob die Kursgröße von drei Studenten, die Kursdauer von zwei Zeitstunden und die Zeitaufteilung für die jeweilige Aufgabenstellung angemessen waren.

\subsubsection{Vorbereitung}

Der Studentenkurs fand in Kabine 8 der unfallchirurgischen Ambulanz der Georg-August-Universität Göttingen statt. Für den Kurs stand den Studenten das Siemens-Ultraschallgerät SONOLINE Elegra zur Verfügung. Zusätzlich wurde für den Zeitraum der Studie das virtusMED-System mit Styropormodell in diesem Raum untergebracht.

Vor Inbetriebnahme des virtusMED-Systems musste die Position des Styroportorsos überprüft und auf das Computersystem abgestimmt werden, um identische Voraussetzungen für jede Gruppe zu erhalten.

Auch die Funktion des Ultraschallgerätes, insbesondere der Sonde $(3.5 \mathrm{C} 40 \mathrm{H}$ Convex), wurde vor Gebrauch getestet, um einen kontinuierlichen Kursablauf zu gewährleisten.

\subsubsection{Gruppe A (erst virtusMED)}

\subsubsection{Einführung des virtusMED-Systems}

Gruppe A erhielt zunächst eine zwanzigminütige Einführung in die Sonographie anhand des virtusMED-Systems. Zu Beginn wurden in aller Kürze die physikalischen Grundlagen der Sonographie sowie die Entstehung des virtusMED-Systems erklärt. Anschließend folgte eine sonographische Demonstration des Bachraumes. Systematisch wurden dabei die Organe in Längs- und Querrichtung mit hilfreichen Erläuterungen dargestellt.

Begonnen wurde mit dem Oberbauchquerschnitt und der Identifikation von Leber, V. cava inferior und Aorta. Durch entsprechendes Kippen des Schallkopfes wurde das Herz mit rechtem und linkem Atrium sowie den jeweiligen Ventrikeln sichtbar. Die Darstellung der V. lienalis und des Pancreas 
folgte. Als nächstes wurde die Leber von subkostal mit den Lebervenen, $\mathrm{V}$. portae und der Gallenblase dargestellt. Von der Flanke aus wurde den Studenten anschließend die rechte Niere in Quer- und Längsrichtung gezeigt und auf den M. iliopsoas hingewiesen. Die großen Gefäße Aorta und V. cava wurden ebenfalls in Längsrichtung zusätzlich mit den Abgängen Truncus coeliacus und A. mesenterica superior demonstriert. Die Darstellung von linker Niere und Milz folgte.

Auf die Bedienung der verschiedenen Funktionen des virtusMED-Systems wurde hingewiesen. Anhand der Milz wurde die Freeze- und Messfunktion eingeführt. Abgeschlossen wurde die Demonstration mit der Darstellung der Harnblase.

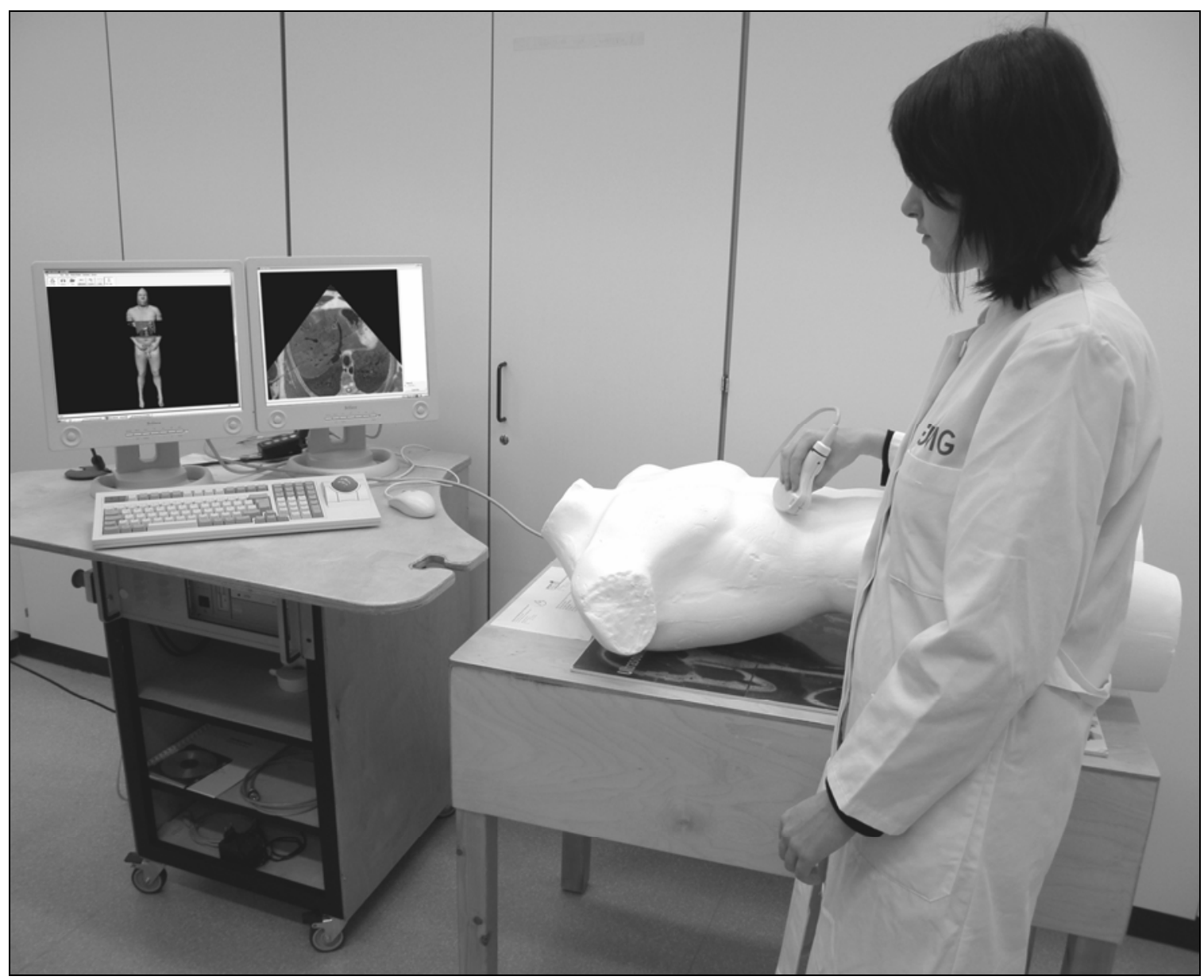

Abbildung 1: virtusMED in der Anwendung: Auf dem linken Monitor erscheint die 3D-Ansicht des virtuellen Patienten und auf dem rechten Monitor die 2D-Ansicht (Ultraschallmodus), die der Position des Schallkopfes entspricht. Der Schallkopf lässt sich auf dem Styroportorso beliebig verschieben. 


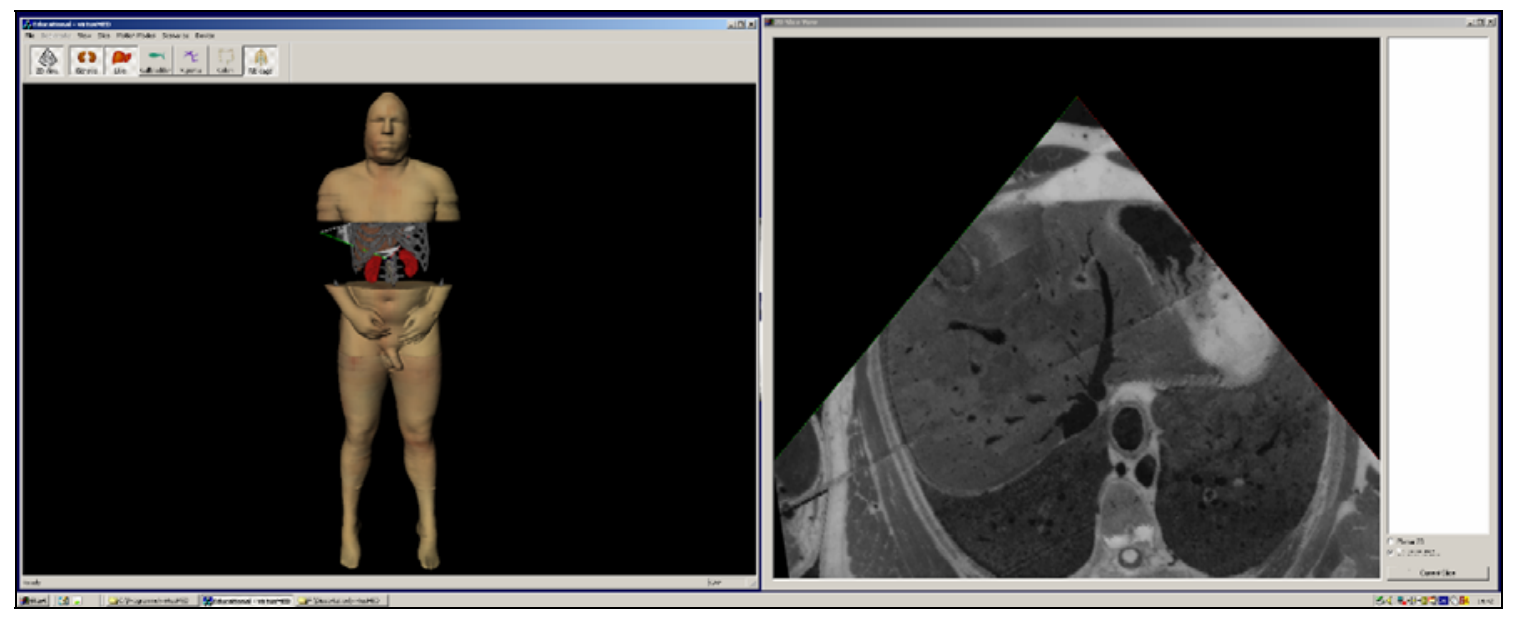

Abbildung 2: virtusMED Ausschnittsvergrößerung der Bildschirmansicht: links im Bild 3DDarstellung des virtuellen Patienten, rechts im Bild 2D-Ansicht (Ultraschallmodus) der Leber von subkostal

\subsubsection{Selbständiges Explorieren des virtusMED-Systems - virtuelle Sonographie}

Die Studenten hatten der Reihe nach die Möglichkeit das virtusMED-System selbständig auszuprobieren. Die Aufgabe bestand darin, dieselben Organe wie in der zuvor gezeigten Demonstration aufzusuchen und darzustellen. Außerdem sollten die verschiedenen Funktionen des virtusMED-Systems exploriert werden, um so eine für jeden Studenten individuell passende Bildschirmoberfläche zu schaffen. Den Studenten wurde mit Absicht kein Zeitlimit für die Übung gegeben. Nach Beendigung der selbständigen Exploration wurde der Schallkopf an die nächste Person weitergegeben. In der Zeit, in der die Studenten nicht aktiv selber schallten, hatten sie die Möglichkeit die virtuelle Untersuchung ihrer Kommilitonen auf den beiden Bildschirmen zu verfolgen.

\subsubsection{Kurztest am virtusMED-System}

An das eigenständige Ausprobieren schloss sich ein Kurztest an. Dieser wurde von den jeweiligen Studenten einzeln, ohne die Anwesenheit der Kommilitonen, durchgeführt.

Die Grundposition des Schallkopfes für die beiden Aufgaben war der Oberbauchquerschnitt. Als erstes wurde die Aufgabe gestellt. Wurde sie verstanden durfte der Student beginnen. Die Zeit bis zur Einstellung des gesuchten Bildes wurde gestoppt. Die Freezetaste wurde für die Studenten 
gedrückt, sobald sie der Meinung waren, das korrekte Bild eingestellt zu haben. Die Aufgabe war nicht bestanden, wenn das Bild falsch eingestellt wurde, oder die falsche anatomische Achse zum Ausmessen gewählt wurde.

Die erste Aufgabe bestand darin, die Aorta im Längsschnitt darzustellen, so dass die Abgänge des Truncus coeliacus und der A. mesenterica superior deutlich sichtbar sind. In diesem Bild sollten die Studenten anschließend die Gefäße benennen können und die Bandscheibe, die dorsal der A. mesenterica superior liegt, in Höhe und Tiefe ausmessen.

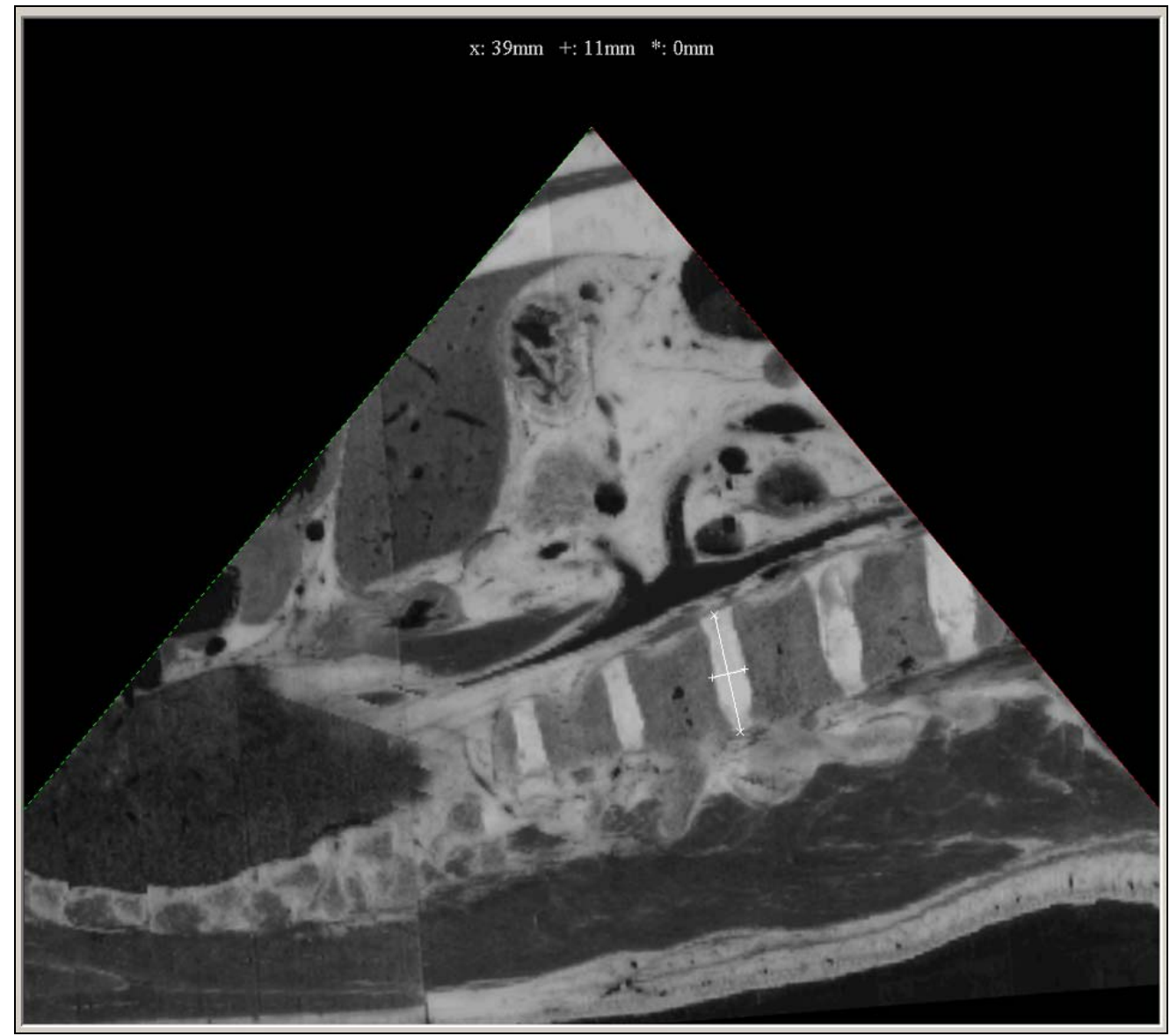

Abbildung 3: Darstellung der Aorta im Längsschnitt mit Abgängen des Truncus coeliacus und der A. mesenterica superior. Ausgemessen ist die dorsal der A. mesenterica superior liegende Bandscheibe in Höhe und Tiefe (Aufgabe 1). 


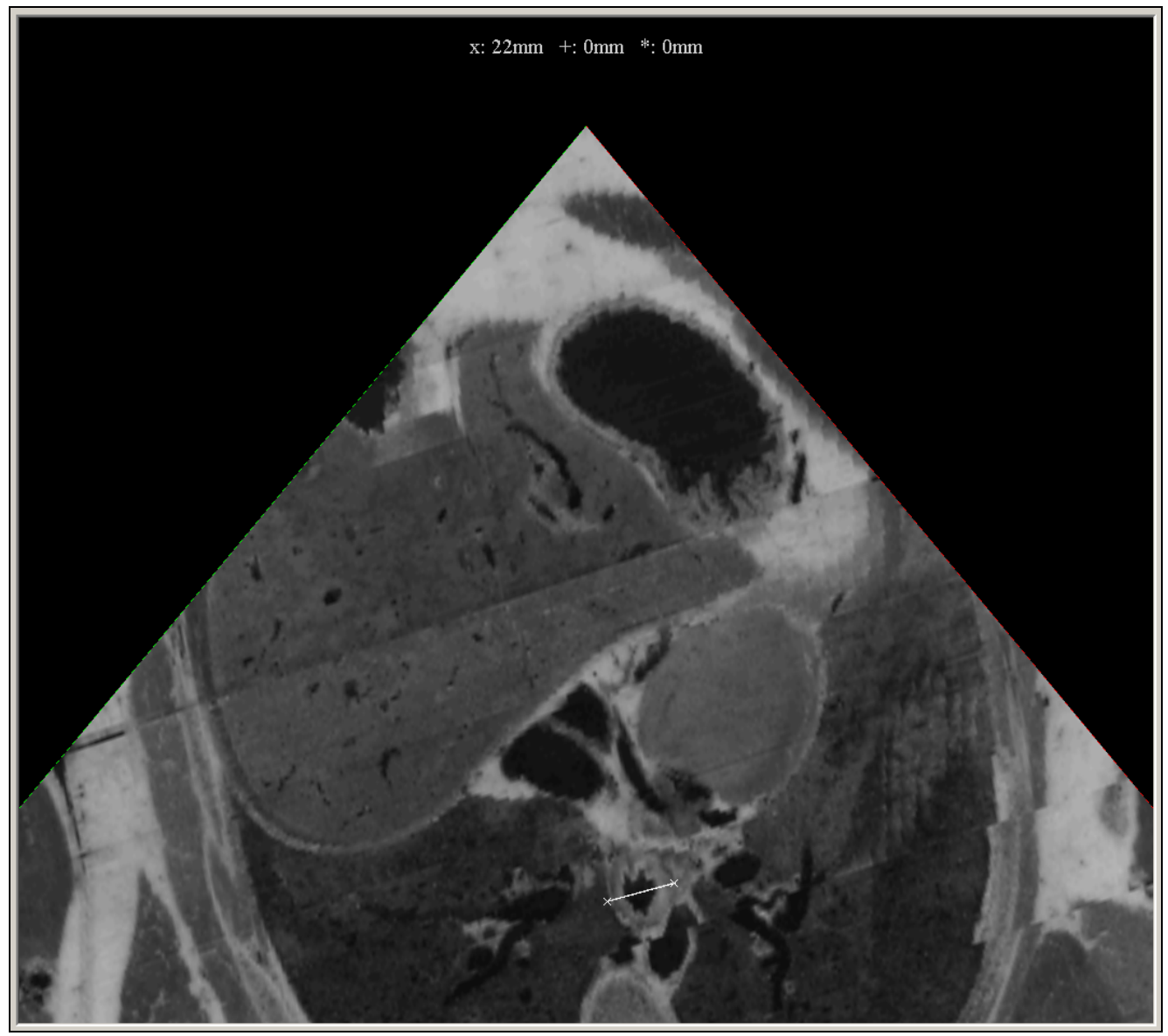

Abbildung 4: Darstellung des Übergangs von V. cava inferior in den rechten Vorhof. Ausgemessen ist der Querdurchmesser des Ösophagus (Aufgabe 2).

Die zweite Aufgabe bestand darin, im Querschnitt den Übergang zwischen V. cava inferior und rechtem Atrium dazustellen. Auf entsprechendem Bild erscheint ebenfalls im Querschnitt der Ösophagus, dessen Außendurchmesser (mit T. muscularis) von den Studenten ausgemessen werden sollte (siehe Abbildung 4).

\subsubsection{Ultraschalluntersuchung einer Testperson - reale Sonographie}

Dem Vortraining mit dem virtusMED-System folgte die reale Untersuchung eines „Patienten“ bzw. einer der beiden Testpersonen.

\subsubsection{Einführung des Ultraschallgerätes (SONOLINE Elegra von Siemens)}

Die Studenten erhielten erneut eine etwa zwanzigminütige Einführung, in der wichtige Funktionen (Freeze- und Messfunktion) erklärt wurden. Zusätzlich erhielten sie Tipps zur Bedienung des Schallkopfes (3.5C40H Convex) und für den Umgang mit Patienten. 
Die Demonstration begann wie zuvor beim virtusMED-System mit dem Oberbauchquerschnitt und der Identifikation von Leber und den großen Gefäßen. In den meisten Fällen ließen sich Teile des Herzens von substernal gut darstellen. Nicht immer war es möglich die Pankreasregion und die V. lienalis eindeutig darzustellen. Durch die charakteristische wellenförmige Doppelpulsation der V. cava inferior und die prägnante Pulsation der Aorta waren die großen Gefäße besonders im Längsschnitt gut abgrenzbar. Von subkostal und interkostal wurde den Studenten die Leber in zwei Ebenen mit ihren Lebervenen, der V. portae und der Gallenblase gezeigt. Zusätzlich wurde innen erklärt, wie sich Gallensteine im Ultraschall darstellen und wie wichtig Bildartefakte zur Erkennung von Strukturen bei der Sonographie sind. Von der rechten Flanke aus folgte die Darstellung der rechten Niere im Längs- und Querschnitt. An dieser Stelle ließ sich besonders gut die Atemverschieblichkeit der Bauchorgane darstellen. Durch langsames Ein- und Ausatmen der Testperson konnten die Studenten beobachten, wie sich die Niere auf dem M. iliopsoas bewegte. Der Versuchsleiter umfuhr auf dem Monitor die Niere mit dem Finger und machte deutlich, wie die Studenten die Niere in Längs- und Querrichtung auszumessen hatten. Als nächstes folgte die Darstellung der Harnblase und im Anschluss daran wurde innen die linke Niere in Längs- und Querrichtung gezeigt. Durch erneutes deutliches Ein- und Ausatmen der Testperson konnte man das Kulissenphänomen der Lunge veranschaulichen. Abschließend wurde die Milz demonstriert und anhand dieser die Messfunktion eingeführt.

\subsubsection{Selbständiges Explorieren am Ultraschallgerät}

Die Studenten sollten als nächstes in Analogie zum virtusMED-System die Strukturen, die innen zuvor gezeigt worden waren, wieder finden und darstellen. Es gab kein Zeitlimit und die Studenten hatten ausreichend Zeit Gefäße, Leber, Niere und Milz aufzusuchen. Besonderer Wert wurde darauf gelegt, dass die Studenten die beiden Nieren in Längs- und Querrichtung richtig darstellten und verstanden, wie man diese Organe in Länge, Breite und Tiefe ausmaß. Die Dimensionen der Niere wurden hierzu vom Versuchsleiter mit den Fingern am Monitor dargestellt. Es wurde erklärte, wie wichtig es sei, die Organe „durchzufächern“, um so die maximale Ausdehnung eines Organs zu ermitteln. 
Nach etwa zwölf Minuten gaben die Studenten den Schallkopf an ihre Kommilitonen weiter. In der Zeit, in der sie selber nicht aktiv schallten, hatten die Studenten die Gelegenheit, die Untersuchung der Kommilitonen auf dem Bildschirm zu verfolgen.

\subsubsection{Kurztest am Ultraschallgerät}

An das eigenständige Ausprobieren schloss sich ein weiterer Kurztest an, der von den jeweiligen Studenten einzeln, ohne die Anwesenheit der Kommilitonen, durchgeführt wurde.

Wurde die gestellte Aufgabe verstanden, durfte der Student beginnen. Die Zeit bis zur Einstellung des Bildes wurde gestoppt. Dokumentiert wurde die Zeit in Minuten und ganzen Sekunden. Zehntelsekunden kleiner als 00:00:50 min wurden abgerundet und größer als 00:00:50 min wurden aufgerundet (Beispiel: 1:15:56 $\min =1: 16 \mathrm{~min})$. Die Freeze-Taste wurde für die Studenten gedrückt, sobald sie der Meinung waren, das korrekte Bild eingestellt zu haben. Die Aufgabe war nicht bestanden, wenn das Bild falsch eingestellt wurde bzw. die falsche anatomische Achse ausgemessen wurde. Länge, Breite und Tiefe wurden in Millimetern mit einer Kommastelle dokumentiert (Beispiel: rechte Niere längs Länge: 95,6 mm).

Die erste Aufgabe bestand darin, die rechte Niere im Längsschnitt darzustellen, so dass anschließend die maximale Länge und Breite ausgemessen werden konnte (siehe Abbildung 6). Die Testpersonen halfen bei dieser Aufgabe meist durch geschicktes Ein- und Ausatmen mit, ohne dass der Student sie darum bitten musste.

Als zweites sollte dieselbe Niere im Querschnitt dargestellt werden (siehe Abbildung 8). Das Bild sollte im Idealfall an der Stelle angehalten werden, an der die Abgänge bzw. Zuflüsse der Nierengefäße zu erkennen sind. Waren die Gefäße nur schwer auszumachen, durfte der Student das Bild an der Stelle anhalten, an der sich nach seinem eigenen Ermessen die Mitte der Niere befand. In diesem Bild sollten die Breite und die Tiefe des Organs ausgemessen werden. 


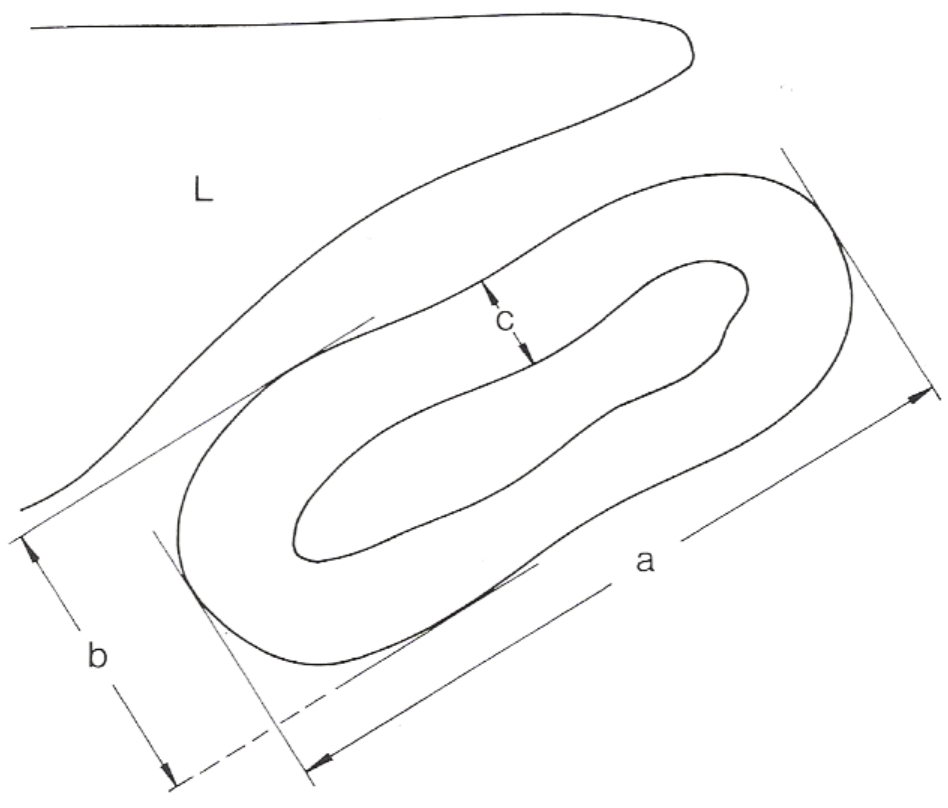

Abbildung 5: Messstrecke der rechten Niere; Schnittführung: sagittaler Längsschnitt, a.p. in der Medio-Klavikularlinie (Vogel und Knierer 1995 S. 166). $a=$ Länge, $b=$ Breite, $c=$

Parenchymdicke

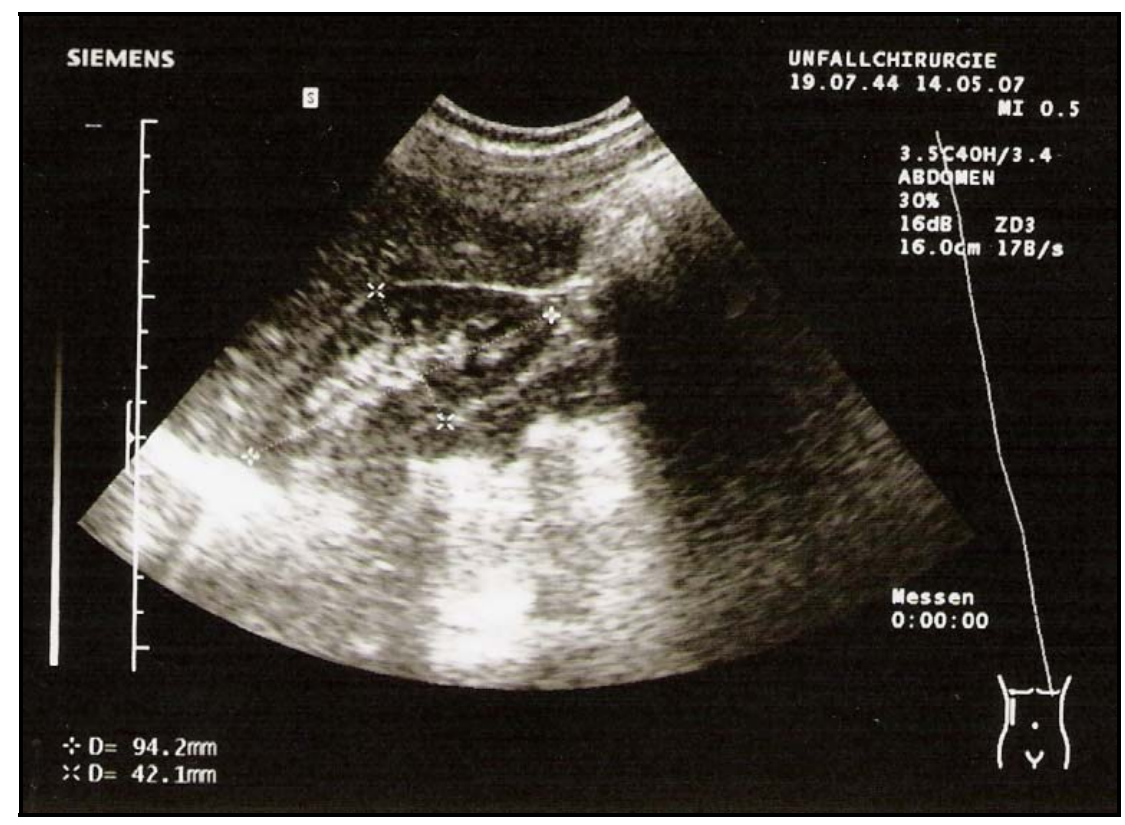

Abbildung 6: Längsschnitt durch die rechte Niere, a.-p. in MedioKlavikularlinie mit Messung der Länge + und Breite $x$ 


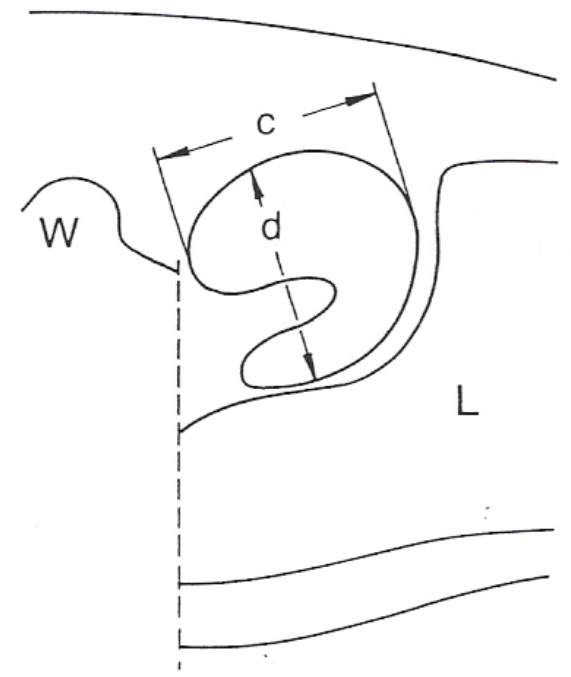

Abbildung 7: Querschnitt durch die rechte Niere, p.-a- (Vogel und Knierer 1995 S. 167 nach Weitzel 1978). $c=$ Breite,$d=$ Tiefe

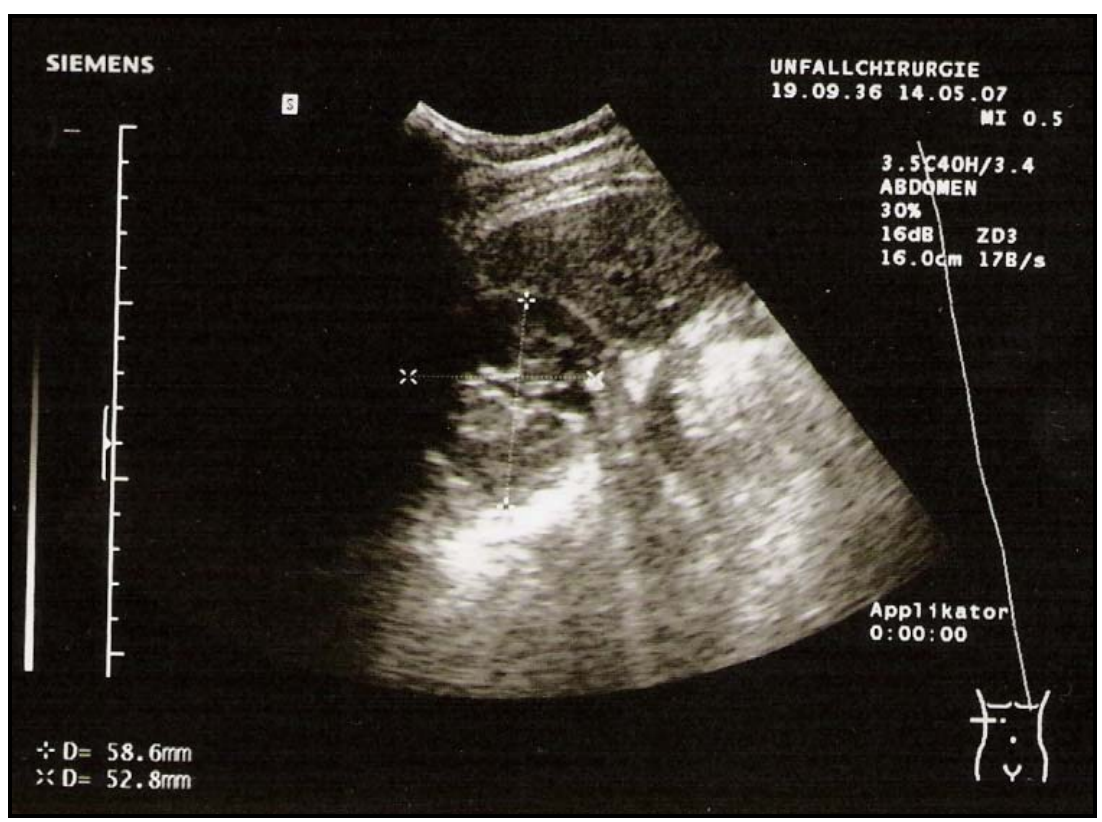

Abbildung 8: Querschnitt durch die rechte Niere, a.-p. mit Messung der Breite $\mathrm{x}$ und Tiefe +

Die dritte und letzte Aufgabe bestand darin, die linke Niere im Längsschnitt darzustellen und ihre maximalen Länge und Breite auszumessen.

Als letztes erhielten die Studenten einen Fragebogen (siehe 8.1), den sie eigenständig auszufüllen hatten. 


\subsubsection{Gruppe B (erst Ultraschall)}

\subsubsection{Einführung des Ultraschallgerätes (SONOLINE Elegra von Siemens)}

Die Gruppe B erhielt als erstes eine zwanzigminütige Einführung in die sonographische Untersuchung in Theorie und Praxis. Physikalische Grundkenntnisse wurden erneuert sowie Tipps zum Umgang mit Patienten und dem Umgang mit dem Schallgerät gegeben. Die sonographische Demonstration verlief wie für die Gruppe A (siehe 3.4.2.5).

\subsubsection{Selbständiges Explorieren am Ultraschallgerät - reale Sonographie} In Analogie zu Gruppe A hatten die Studenten der Gruppe B ebenfalls etwa 12 Minuten Zeit, um eine Testperson sonographisch zu untersuchen, Funktionen des Ultraschallgerätes auszuprobieren und Fragen zu stellen.

\subsubsection{Kurztest am Ultraschallgerät}

Die Studenten der Gruppe B wurden ebenso einzeln geprüft und erhielten dieselben Aufgaben wie die Gruppe A (siehe 3.4.2.7).

\subsubsection{Einführung des virtusMED-Systems}

Als nächstes wurden die Studenten mit dem virtusMED-System vertraut gemacht. Sie erhielten dieselbe Demonstration der Bauchorgane des virtuellen Patienten wie die Gruppe A (siehe 3.4.2.1).

\subsubsection{Selbständiges Explorieren des virtusMED-Systems - virtuelle Sonographie}

Auch die Studenten der Gruppe B sollten eigenständig die zuvor gezeigten Organe auffinden und darstellen. Ein Zeitlimit gab es hierfür nicht. Im Durchschnitt gaben die Studenten den Schallkopf nach etwa fünf Minuten an ihre Kommilitonen weiter und verfolgten anschließend deren virtuelle Untersuchung. 


\subsubsection{Kurztest am virtusMED-System}

An das selbständige Erproben des virtusMED-Systems schloss sich derselbe Test wie für die Gruppe A an (siehe 3.4.2.3).

Zum Schluss bekamen die Studenten der Gruppe B den identischen Evaluationsbogen über das virtusMED-System, der eigenständig auszufüllen war.

\subsection{Verwendete Software - virtusMED}

virtusMED ist eine Software, die an der Technischen Universität Braunschweig entwickelt wurde, um Studenten und Ärzten einen besseren Einblick in den menschlichen Körper zu geben. Das Computerprogramm basiert auf fotografischen Schnittbildern, CT und MRT Daten des "Visible Human Projektes“ (National Library of Medicine, Bethesda, USA).

Dieses System war ursprünglich als Lern- und Orientierungshilfe für das Verstehen der menschlichen Anatomie entwickelt worden, kann seit neuestem aber auch zur Simulation von Sonographieuntersuchungen eingesetzt werden. Nach Vorstellungen des Betreuers dieser Arbeit wurde die Software erweitert. Durch Umschalten vom Farbmodus in den Graustufenmodus entfallen Informationen. Die Eindringtiefe wird durch Simulation in der Weise dargestellt, dass die Schärfenebene verändert wird. Der Student benutzt eine virtuelle Untersuchungssonde (Analogie: Ultraschallsonde), um in Echtzeit beliebige Schnittbilder eines virtuellen Menschen zu erzeugen. Neben einem 2D-Bild (Ultraschallmodus) kann parallel eine 3D-Szene auf dem Computermonitor dargestellt werden. Als Orientierungshilfe dient ein künstlicher Torso aus Styropor. 


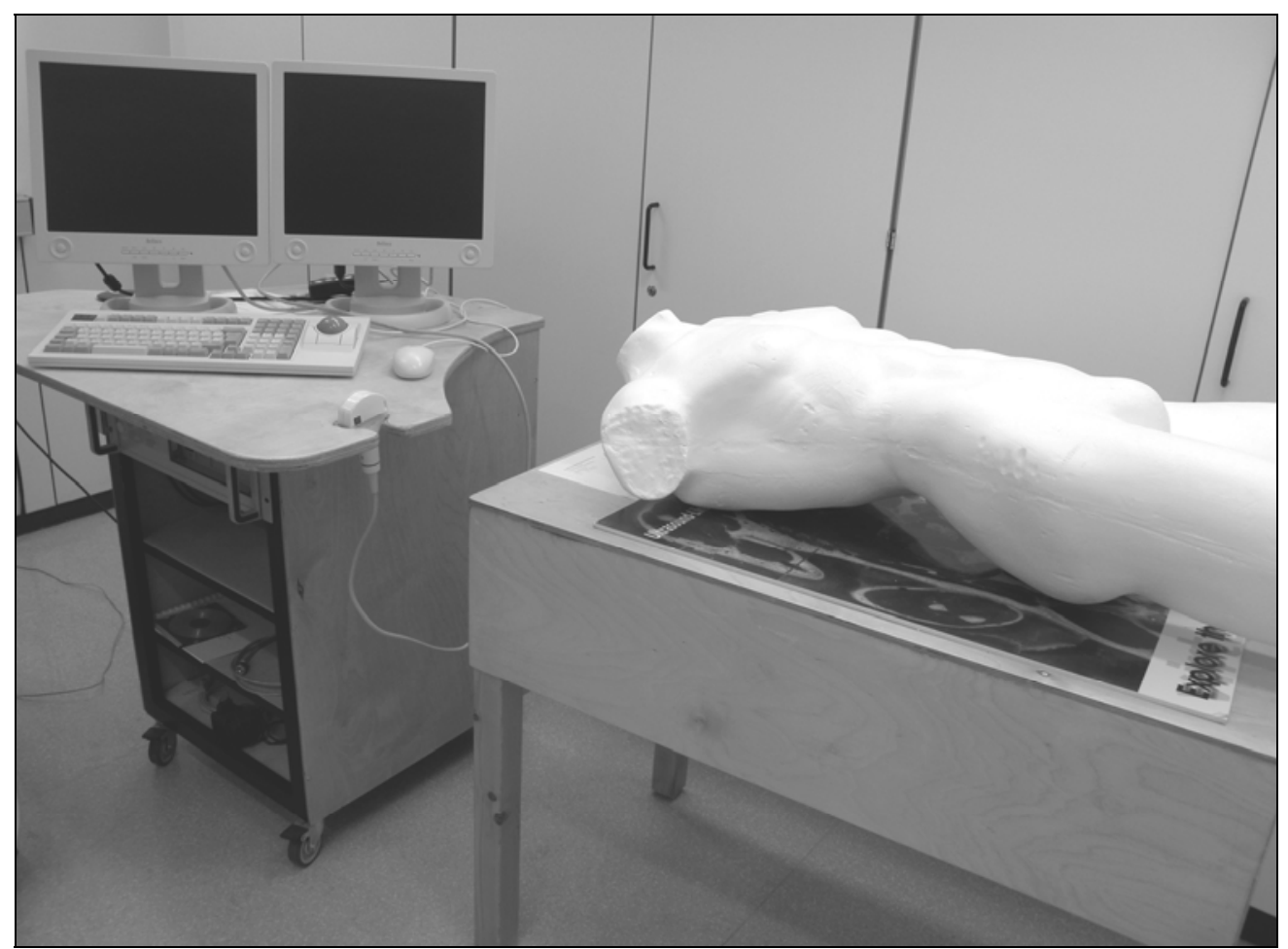

Abbildung 9: Versuchsaufbau: zwei Bildschirme, Tastatur, Maus, Sonde und Styroportorso bzw. Poster

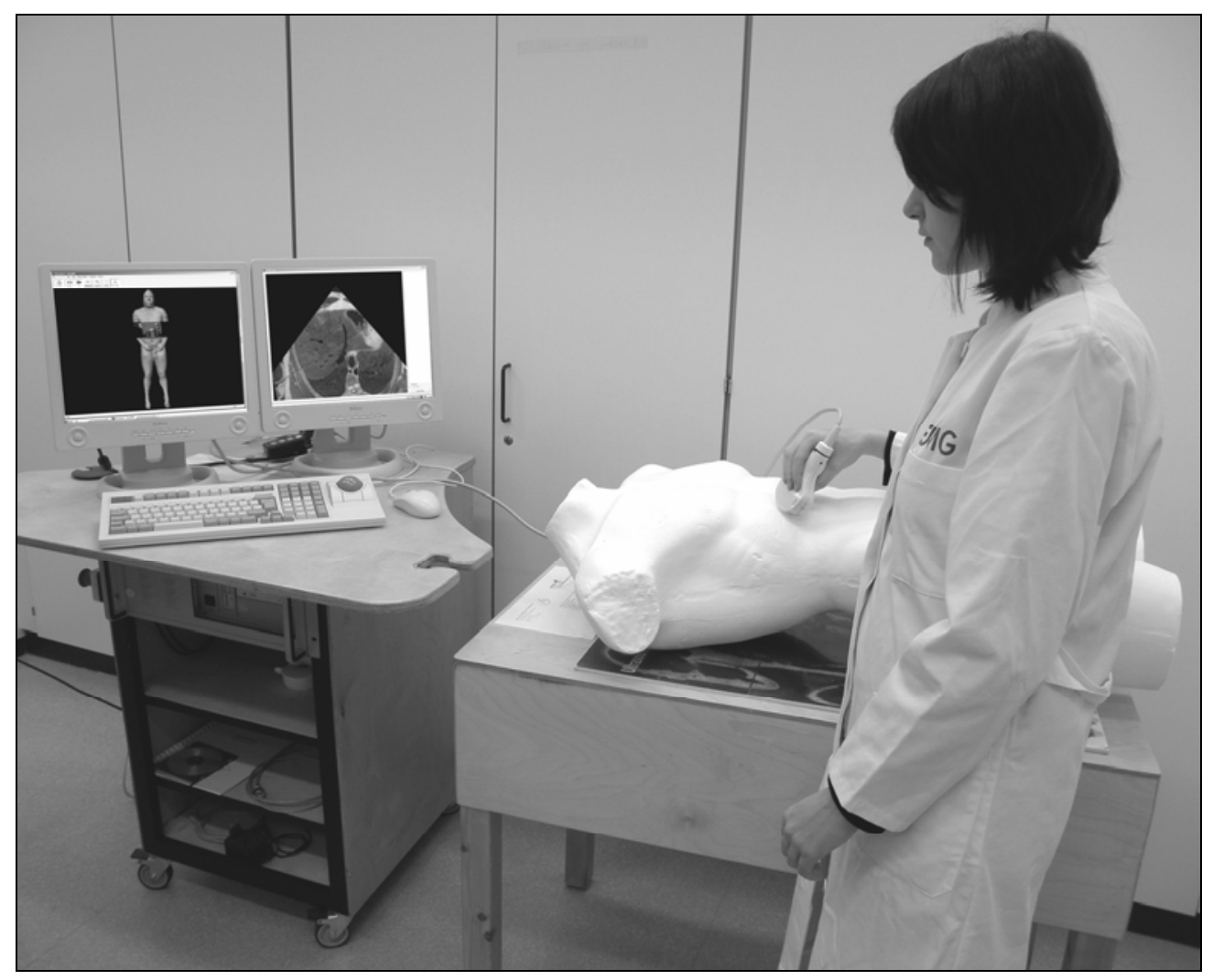

Abbildung 10: virtusMED in der Anwendung (= Abbildung 1) 


\subsubsection{Bedienung des virtusMED-Systems}

\subsubsection{Programmstart}

Durch einen Doppelklick auf das Symbol virtusMED.exe öffnet sich nach einigen Sekunden ein schwarzes Fenster. Man wird aufgefordert, die gewünschten Daten zu laden (Please load volume data). Mit einem Einfachklick auf den Button Scenarios (der sich in der Menüleiste befindet) öffnet sich eine Leiste, aus der verschiedenen Körperabschnitten ausgewählt werden können. Um eine virtuelle Sono-Abdomen-Untersuchung durchzuführen, wird am besten auf Torso und anschließend auf Overview geklickt. Nach ein paar Sekunden erscheint die Startansicht mit dreidimensionalem Patienten.

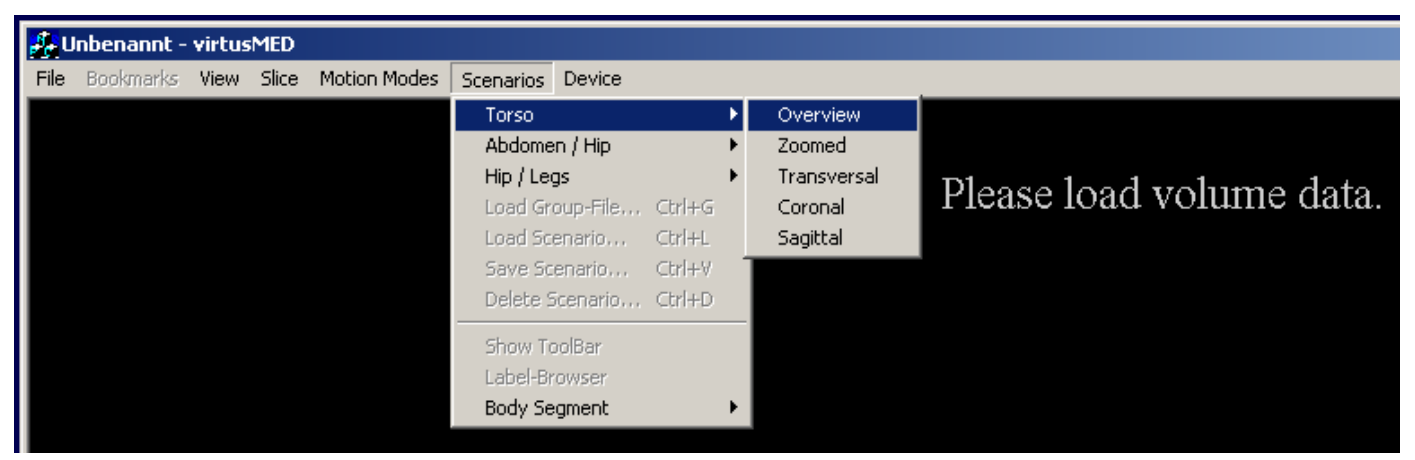

Abbildung 11: Anweisung zum Laden der Volumendatei

Um das zusätzliche Ultraschallbild zu erhalten, muss auf der neu erscheinenden Menüleiste auf 2D View geklickt werden. Ein zweites Fenster öffnet sich. Zur besseren Übersicht stehen zwei Bildschirme zur Verfügung, so dass die Ultraschallansicht auf den rechten Bildschirm verschoben werden kann. Beide Fenster können maximiert werden und füllen so den gesamten Bildschirm (siehe Abbildung 12 und Abbildung 13) aus.

Vor jeder virtuellen Exploration sollte die Position des Styroportorsos überprüft werden. Am besten gelingt dies, indem die Untersuchungssonde unterhalb des Xyphoids im Querschnitt gehalten wird und der Torso anhand der 3D-Ansicht in Position gerückt wird. 


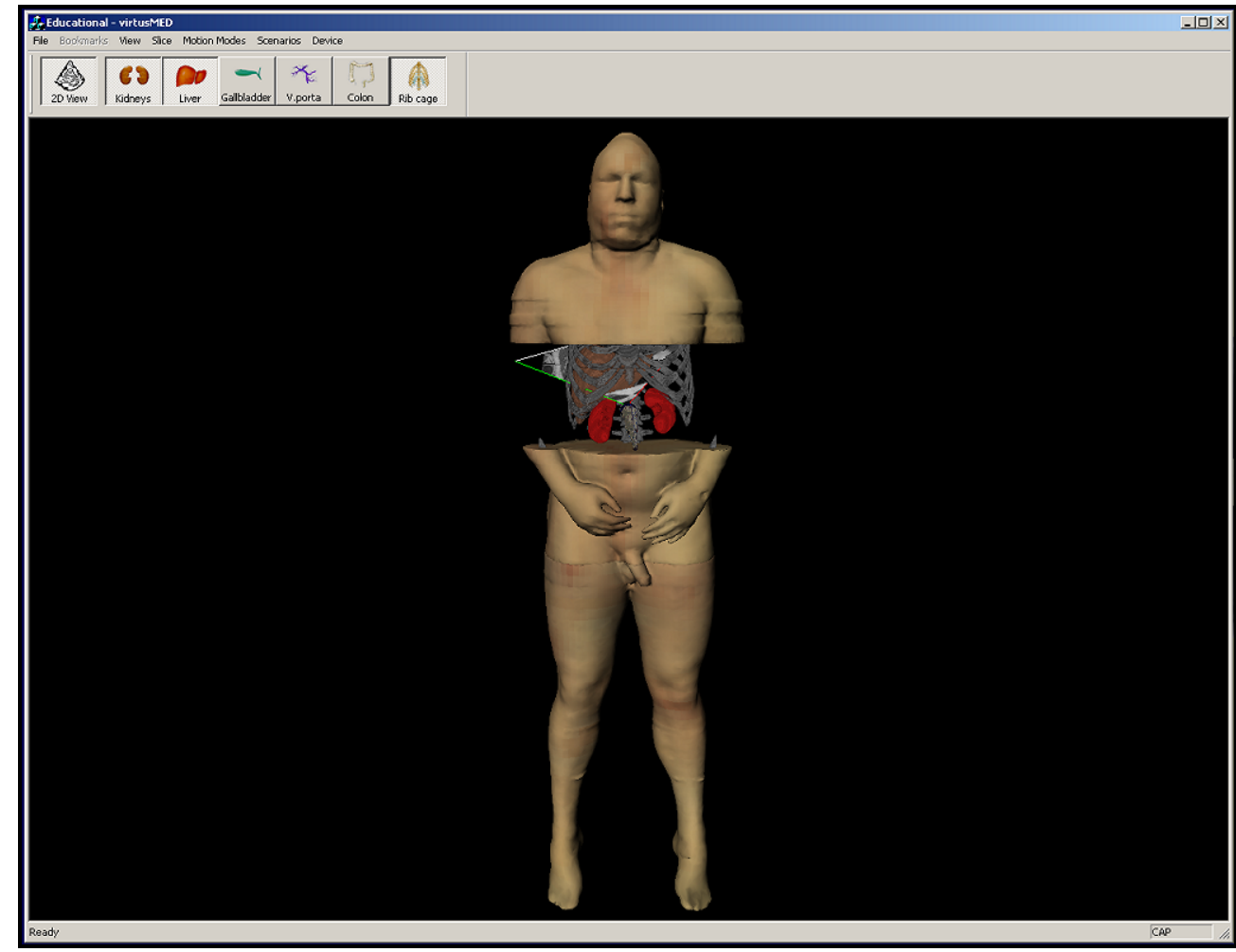

Abbildung 12: Abbildung des linken Bildschirms mit Übersichtsdarstellung des virtuellen Patienten (3D-Ansicht). Leber (braun) und Niere (rot) erscheinen als zusätzliche Organe im Untersuchungsbereich. Die Untersuchungssonde und der Schallsektor sind ebenfalls abgebildet.

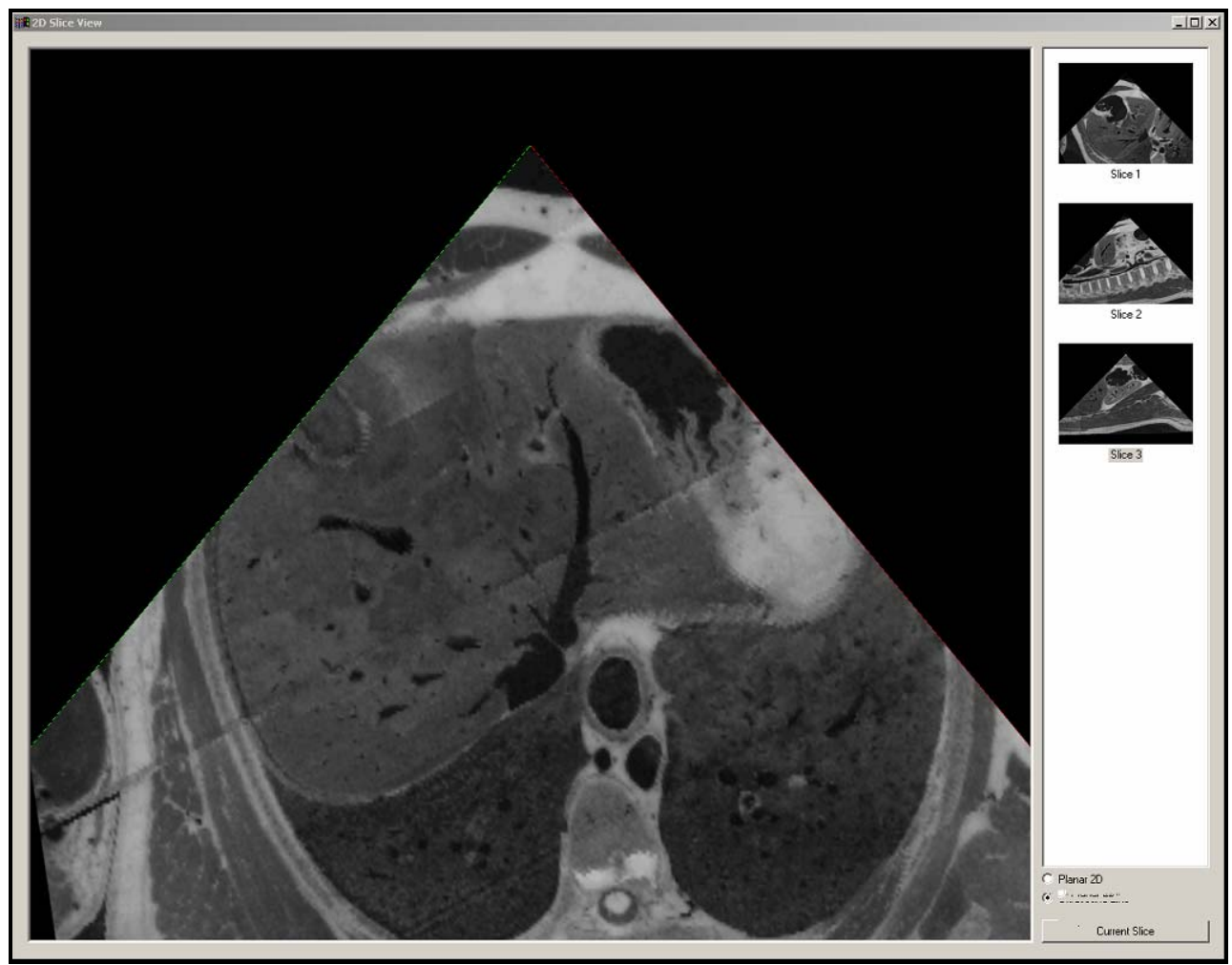

Abbildung 13: Abbildung des rechten Bildschirms. Dargestellt ist ein "Ultraschallbild“, das der Position der Untersuchungssonde in Abbildung 12 entspricht. In der rechten Leiste des Fensters erscheinen gespeicherte Bilder, auf die zu einem späteren Zeitpunkt zurückgegriffen werden kann. 


\subsubsection{Die virtuelle Untersuchung}

Die für das virtusMED-System verwendete Untersuchungssonde entspricht in Aussehen und Funktion in etwa einem Konvexscanner, der normalerweise bevorzugt für die Abdomenuntersuchung verwendet wird. Mithilfe dieser Sonde kann der virtuelle Patient untersucht werden, indem sie an gewünschter Position über der Styroporpuppe bewegt wird. Auf dem linken Bildschirm (3D-Ansicht) kann der Verlauf der Ultraschallwellen nachvollzogen werden. Der rechte Bildschirm (2D-Ultraschallansicht) gibt das entsprechende "Ultraschallbild“ wieder, wobei zu bemerken ist, dass es sich aufgrund der Datenquelle (Fotodaten) der Software nicht um ein wirkliches Ultraschallbild handelt.

Die rote und grüne Linie, die den Sektor der 2D-Ansicht begrenzt, entspricht den Linien des Schallsektors in der 3D-Ansicht. Mit ihrer Hilfe gewinnt der Untersucher eine bessere Orientierung der dargstellten Seiten (links und rechts, bzw. oben und unten).

\subsubsection{Weitere Funktionen}

Die Software virtusMED besitzt zusätzliche hilfreiche Funktionen, die den Studenten die Orientierung bei der Sonographie erleichtern sollen.

\subsubsection{Der virtuelle Patient (3D-Ansicht)}

Der virtuelle Patient lässt sich mit gedrückt gehaltener linker Maustaste in beliebige Positionen manövrieren. Einige Studenten bevorzugen die Frontalansicht, andere wiederum mögen es lieber, wenn der virtuelle Patient horizontal, gleich dem Styropormodell, liegt.

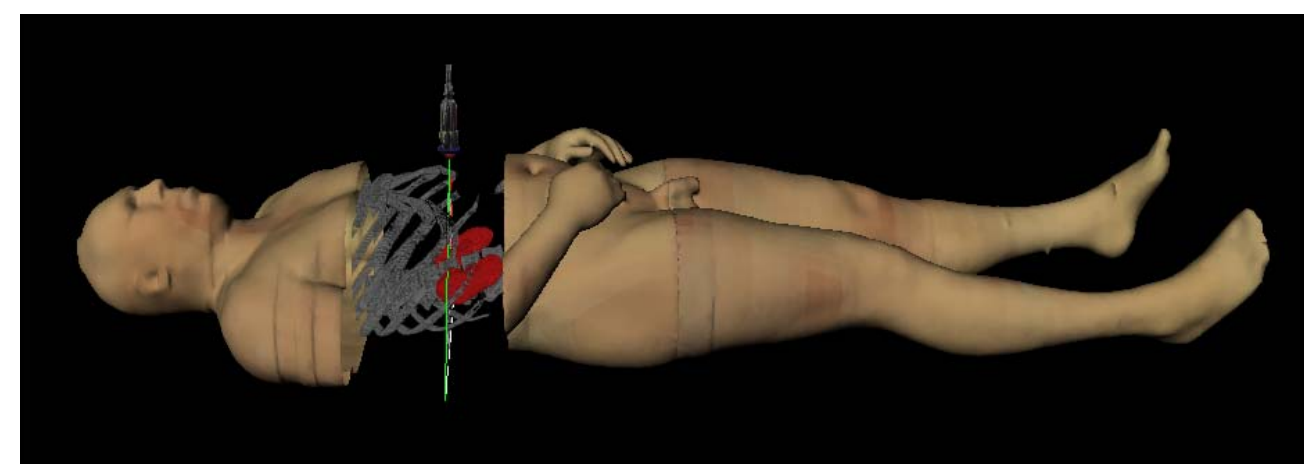

Abbildung 14: Horizontalansicht des virtuellen Patienten (3D-Ansicht) 


\subsubsection{Organe hinzufügen}

Durch Einfachklick auf einen Organ-Button aus der Menüleiste wird dem virtuellen Patienten ein dreidimensionales Modell der entsprechenden anatomischen Struktur hinzugefügt. Niere, Leber, Gallenblase, Portalgefäßsystem und Colon lassen sich separat einblenden. Dies kann besonders von Nutzen sein, wenn die Lage eines dieser Organe detailliert studiert werden möchte.

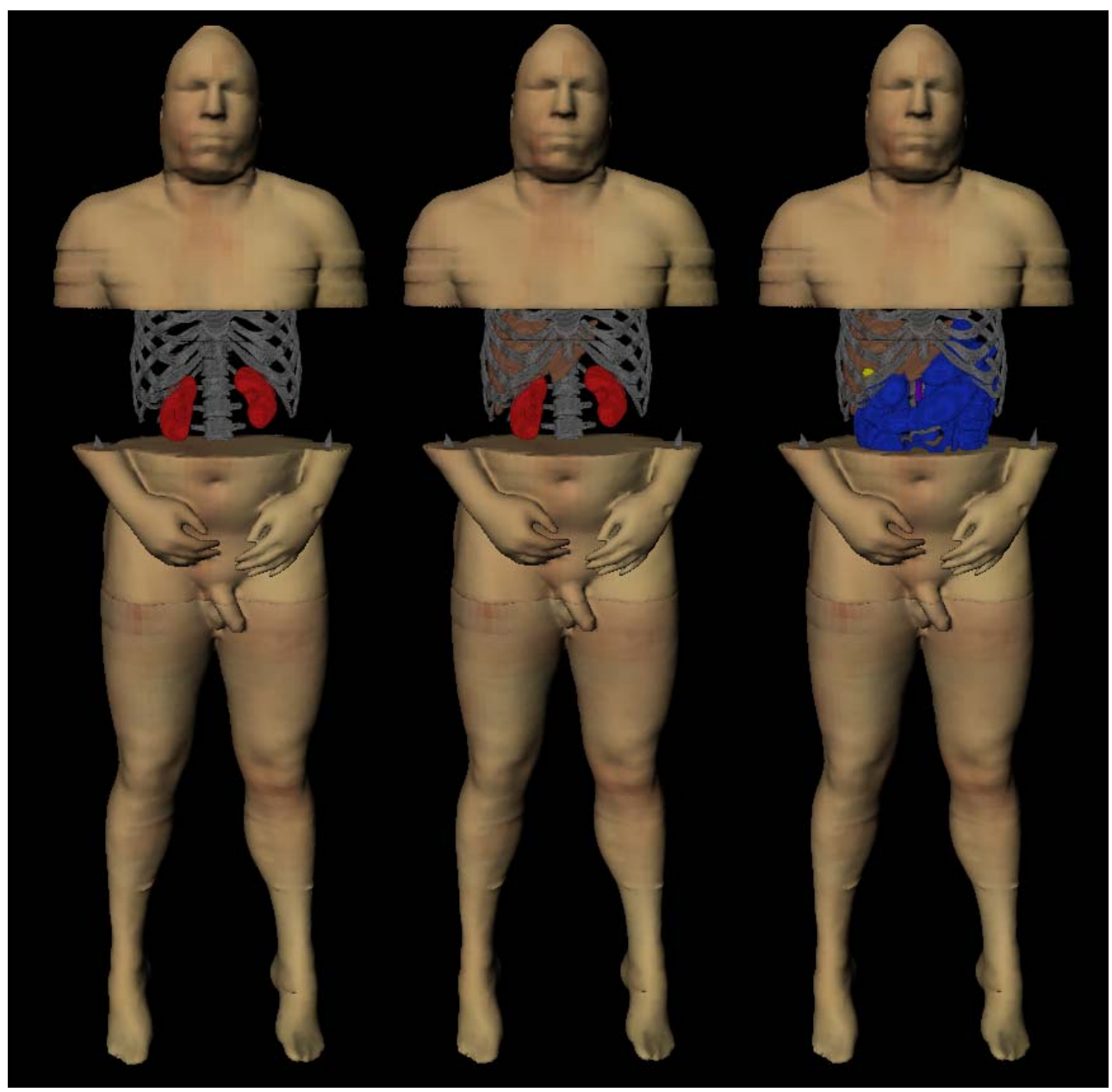

Abbildung 15: linke Darstellung nur mit Nieren (rot), mittlere Darstellung mit zusätzlicher Leber (braun), rechte Darstellung mit hinzugefügtem Colon (blau)

\subsubsection{Organe benennen}

Besteht Unsicherheit bei der Benennung bestimmter Strukturen, genügt es mit der Maus auf entsprechende Struktur in der 2D Ansicht zu fahren und es erscheint der englische Name dieser Struktur im oberen Teil des Fensters. Diese Funktion ist zurzeit nur für größere Strukturen verfügbar. 


\subsubsection{Freeze- und Messfunktion}

Mit Hilfe der Leertaste kann eine bestimmte Bildeinstellung angehalten werden. Bis zu 11 Bilder können so in der breiteren Leiste des rechten Fensters (2D Ansicht) gespeichert werden. Die Position des Schallkopfes wird in der 3D Ansicht ebenfalls gespeichert.

Dies gibt einem die Möglichkeit, auf Bilder zurückzugreifen und gewünschte Strukturen auszumessen. Mit Hilfe der Maus lassen sich bis zu drei Strecken ermitteln. Der gewünschte Anfangspunkt wird durch einmaliges Klicken mit der linken Maustaste angezeigt. Durch erneutes Klicken der linken Maustaste am Endpunkt wird dieser gespeichert. Beide Punkte werden mit einer Geraden verbunden. Die ermittelte Strecke wird in Millimetern angegeben und erscheint im oberen Abschnitt des Fensters.

Um zum Untersuchungsmodus zurückzukehren, muss der Button Current Slice rechts unten im Fenster einmalig gedrückt werden.

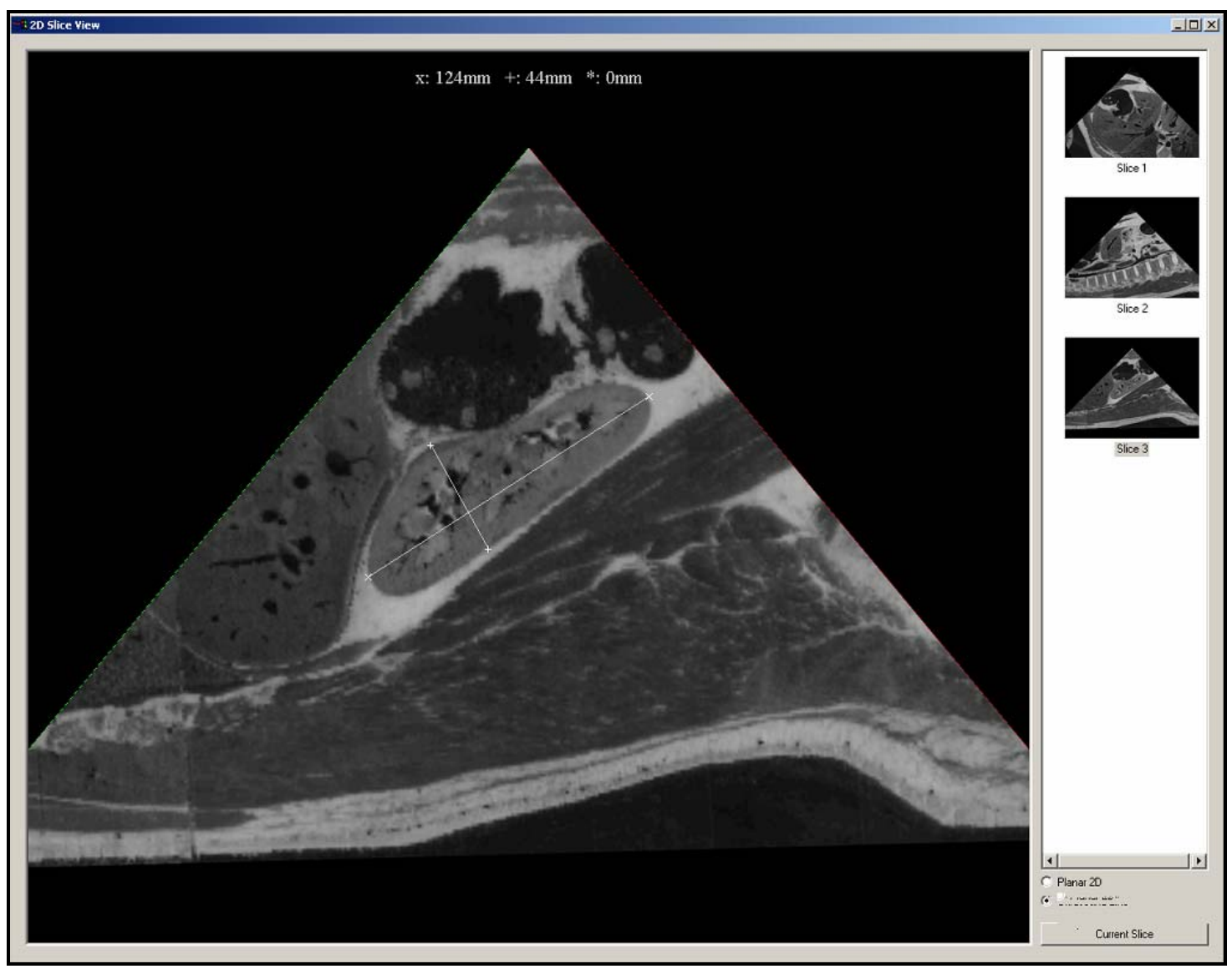

Abbildung 16: „Ultraschallbild“ mit ausgemessener rechter Niere (Länge 124mm, Breite $44 \mathrm{~mm})$. Rechts in der Leiste befinden sich weitere, zuvor gespeicherte Bilder, auf die später zurückgegriffen werden kann und die ebenfalls ausgemessen werden können. 


\subsubsection{Tiefeneinstellungen}

Über den Button Slice können die Schichtparameter und damit die Eindringtiefe verändert werden. Der Sektor (Angle) sowie die Tiefe (Depth) können durch Hinund Herschieben der Balken variiert werden. Zur besseren Übersicht waren der Sektor sowie die Tiefe für die Studenten maximal eingestellt. Die Studenten nahmen an dieser Einstellung meist keine Änderung vor.

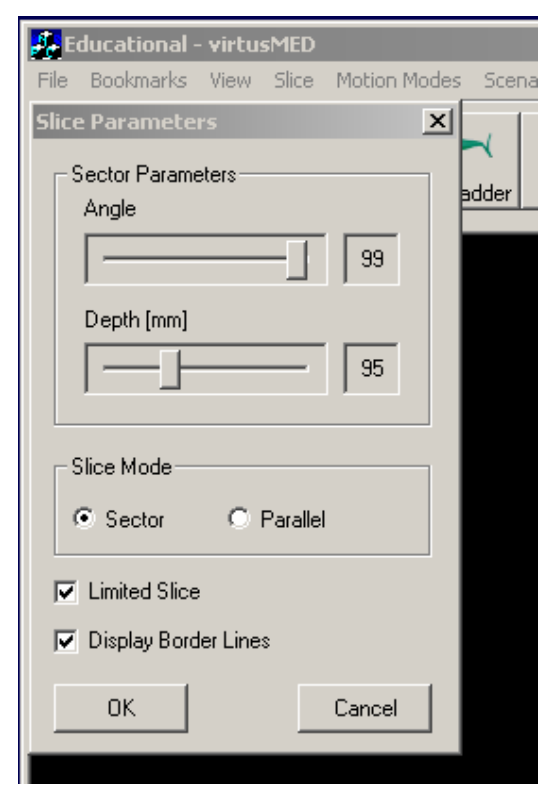

Abbildung 17: Anweisung zum Verstellen der Schichtparameter (Slice Parameters)

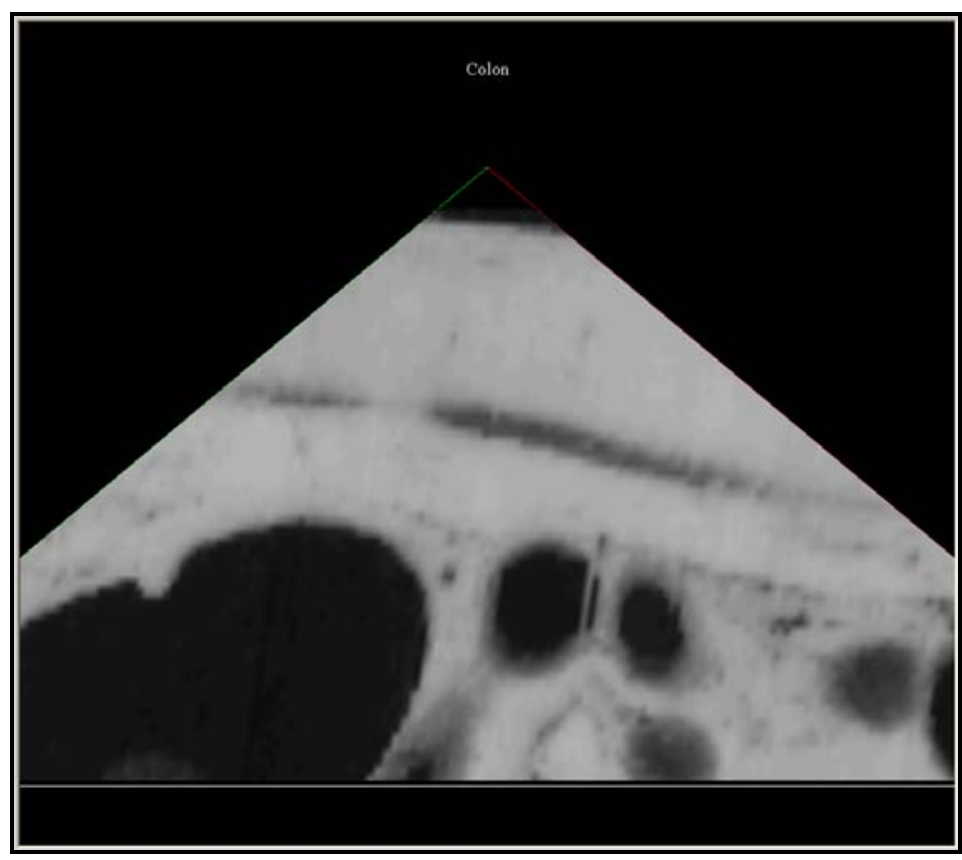

Abbildung 18: 2D-Ansicht („Ultraschallmodus") mit geringerer Tiefe. Unscharf sind die Fett- und Muskelschicht sowie Teile des angeschnittenen Darms zu erkennen. 


\subsection{Erstellung des Fragebogens}

Mit dem selbst konzipierten Fragebogen (siehe 8.1) sollte zum einen der Einsatz des virtusMED-Systems in der sonoraphischen Lehre evaluiert werden und zum anderen der Gebrauch dieses Systems bewertet werden. Zusätzlich wurden Angaben zu PC- und Sonographieerfahrung abgefragt.

Der Fragebogen enthält insgesamt 14 Fragen, mit denen gezielt abgeklärt wurde, ob bestimmte Funktionen wie z.B. die 3D-Ansicht, die Messfunktion oder der Gebrauch des Styropormodells hilfreich im Hinblick auf die Sonographie sind. Offene Fragen lassen dem Studenten die Möglichkeit, in eigenen Worten Vor- und Nachteile des Programms zu benennen sowie Anregungen zur Verbesserung zu geben.

\subsection{Dokumentation, Statistik und Auswertung}

Alle demographischen, messtechnischen und durch den Fragebogen ermittelten Daten wurden in tabellarischer Form mit Windows Microsoft Excel Version 2002 aus Office $X P ®$ festgehalten. Frei formulierte Antworten der Studenten wurden in Windows Microsoft Word Version 2002 sowie in Windows Microsoft Excel Version 2002 aus Office $\mathrm{XP} \circledast$ gespeichert.

Die Auswertung wurde mit Hilfe der deskriptiven Statistik vorgenommen. Die Ergebnisse werden im Ergebnisteil teils graphisch, teils tabellarisch sowie zum Teil im Text mit Angaben folgender Parameter präsentiert:

- Prozentanteil des getesteten und befragten Gesamtkollektivs oder einzelner Untergruppen der jeweiligen Kollektive

- Mittelwert mit oder ohne Standardabweichung

- Medianwert

- Kleinster und größter Wert (= Spannweite)

- Prozentuale Abweichung vom Referenzwert (= vom Versuchsleiter ermittelter Mittelwert).

Die Messergebnisse mussten für die statistische Begutachtung nicht neu kodiert werden. 
Zur Prüfung von Unterschieden zwischen den Studentengruppen A und B sowie Untergruppen (z.B. Sonographievorkenntnisse vs. keine Vorkenntnisse) wurden t-Tests und Varianzanalysen mit Hilfe des Computerprogramms SAS® Version 8.02 durchgeführt. Das Signifikanzniveau wurde dabei bei 5\% angesetzt.

Das Zusammentragen aller Ergebnisse wurde schließlich durch das WindowsProgramm Microsoft Word Version 2002 aus Office XP® koordiniert.

\subsubsection{Referenzwert / Goldstandard}

Die Messergebnisse der einzelnen anatomischen Strukturen, die die Studenten selbständig ermittelten, wurden mit denen vom Versuchsleiter in Beziehung gesetzt.

Um die intraindividuelle Variabilität bzw. die physiologische Standardabweichung beim Ausmessen von Organen zu erhalten, wurden die Nieren der Testpersonen an jedem Untersuchungstag ebenfalls vom Versuchsleiter in ihren Ausmaßen erfasst.

Der Versuchsleiter erwarb sein theoretischen und praktischen Kenntnisse über die Sonographie zum einen durch das Medizinstudium, zum größten Teil jedoch durch das Selbststudium entsprechender Literatur und einer zweiwöchigen Sonographie-Famulatur in der Klinik für Allgemeinchirurgie der Georg-AugustUniversität Göttingen (12.02. - 23.02.2007). Während dieser Zeit lag der Schwerpunkt der Ausbildung in der sonographischen Diagnostik und der postoperativen Verlaufskontrolle allgemeinchirurgischer Patienten sowie Krankheitsbildern aus dem Bereich chirurgischer Notfälle, chirurgischer Onkologie und Transplantationschirurgie. Am Ende der Famulatur bereitete es dem Versuchsleiter keinerlei Probleme eine Abdomensonographie selbständig durchzuführen. Die vom Versuchsleiter erhobenen Messwerte wurden als Referenzwerte verwendet, auf die sich die studentischen Messergebnisse beziehen. 


\subsection{Ziel der Arbeit}

Ziel der Arbeit ist herauszufinden, ob bzw. in welchem Ausmaß das PCProgramm virtusMED einen Einfluss auf das Erlernen der Ultraschalluntersuchung bei Studenten hat und ob es sinnvoll ist, dieses künftig in die Lehre zu integrieren. Dazu werden die Messergebnisse der einzelnen Studenten aus Gruppe A und B miteinander verglichen sowie der Fragebogen analysiert.

Folgende Arbeitshypothesen ergeben sich:

1. Studenten der Gruppe A, die ein Vortraining mit dem virtusMED-System bekommen, sind anschließend in der Lage Organe mit konventionellem Ultraschallgerät genauer auszumessen.

2. Studenten der Gruppe A, die ein Vortraining mit dem virtusMED-System erhalten, sind anschließend in der Lage mit konventionellem Ultraschallgerät Organe schneller auszumessen.

3. Studenten der Gruppe A, die ein Vortraining mit dem virtusMED-System erhalten, können sich anschließend besser bei der Sonographie orientieren.

4. Studenten der Gruppe A, die ein Vortraining mit dem virtusMED-System erhalten, kommen mit der Handhabung der Ultraschallsonde und dem Umgang des Ultraschallgerätes besser zurecht.

Mittels objektiver Messungen (z.B. Länge der Niere) soll herausgefunden werden, ob sich die Hypothesen 1 und 2 bestätigen.

Die Hypothesen 3 und 4 beschreiben das subjektive Befinden der Studenten, das mit Hilfe der Fragebögen analysiert wird.

Zusätzlich soll mittels des Fragebogens herausgefunden werden, wie die Studenten die einzelnen Komponenten des virtusMED-System bewerten, ob sie einen Einsatz in der Lehre für sinnvoll erachten und an welcher Stelle das virtusMED-System möglicherweise verändert bzw. optimiert werden sollte. 


\section{Ergebnisse}

\subsection{Charakterisierung des Gesamtkollektivs $(n=69)$}

Die Studie befasst sich mit einem Gesamtkollektiv von 69 Studenten im Alter zwischen 21 und 32 Jahren, die an einem zweistündigen Ultraschallkurs im Sommersemester 2007 an der Universitätsmedizin Göttingen Georg-AugustUniversität teilnahmen.

Dieses Gesamtkollektiv wurde auf zwei Gruppen (Gruppe A - erst virtusMED n=34 und Gruppe B - erst Sono $n=35$ ) bzw. vier Untergruppen (Gruppe A Testperson $_{1} \mathrm{n}=14$, Gruppe A Testperson ${ }_{2} \mathrm{n}=20$, Gruppe B Testperson ${ }_{1} \mathrm{n}=18$, Gruppe B Testperson $n_{2} n=17$ ) aufgeteilt. Im Folgenden werden diese beiden Gruppen (Gruppe A und B) miteinander verglichen.

\subsubsection{Alter, Semesteranzahl und Geschlechterverteilung}

Von den 69 Studenten waren 54 weiblichen Geschlechts (78,26\%) und 15 männlichen Geschlechts (21,74\%).

Das Durchschnittsalter aller 69 Studenten lag zum Zeitpunkt der Studie bei $22,52 \pm 1,94$ Jahren.

57 der 69 Studenten $(82,61 \%)$ stammten zum Studienzeitpunkt aus dem ersten klinischen Semester, 7 Studenten (10,14\%) aus dem zweiten klinischen Semester und 5 Studenten $(7,25 \%)$ aus dem dritten klinischen Semester.

Die Alters- und Geschlechtsverteilung in den verschiedenen Gruppen ist der folgenden Tabelle zu entnehmen:

Tabelle 1: Alters- und Geschlechtsverteilung im Gesamtkollektiv, in der Gruppe A (erst virtusMED) Testperson ${ }_{1}$ und Testperson ${ }_{2}$ und in der Gruppe B (erst Sonographie) Testperson 1 und Testperson ${ }_{2}$

\begin{tabular}{|l|c|c|c|c|c|}
\hline Studenten & \multirow{2}{*}{ Anzahl } & \multicolumn{2}{|c|}{ Alter } & \multicolumn{2}{c|}{ Geschlecht } \\
\cline { 3 - 6 } & & Mittelwert & Standardabweichung & Männer & Frauen \\
\hline Gesamtkollektiv & 69 & 22,52 & 1,94 & 15 & 54 \\
\hline $\begin{array}{l}\text { Gruppe A (erst virtusMED) } \\
\text { Testperson }\end{array}$ & 14 & 22,21 & 0,80 & 2 & 12 \\
\hline $\begin{array}{l}\text { Gruppe A (erst virtusMED) } \\
\text { Testperson }\end{array}$ & 20 & 23,05 & 3,17 & 2 & 18 \\
\hline $\begin{array}{l}\text { Gruppe B (erst Sono) } \\
\text { Testperson }\end{array}$ (erst Sono) & 18 & 22,22 & 0,81 & 6 & 12 \\
\hline $\begin{array}{l}\text { Gruppe B } \\
\text { Testperson }\end{array}$
\end{tabular}




\subsubsection{Erfahrungen am PC}

Von 69 Studenten gaben 58 Studenten (84,06\%) an, Textverarbeitungserfahrung am Computer zu haben. Alle 69 Studenten (100\%) besitzen einen eigenen PC und haben Erfahrung im Umgang mit Emails. Interneterfahrungen haben 68 von 69 Studenten (98,55\%). 4 Studenten (5,80\%) geben an, Erfahrungen im Bereich von Programmierung zu besitzen.

Folgende Tabelle veranschaulicht die verschiedenen Erfahrungen der Studenten im Umgang mit dem Computer:

Tabelle 2: Erfahrungen der Studenten am PC im Gesamtkollektiv, bei Männern und Frauen

\begin{tabular}{|l|c|c|c|c|c|c|}
\hline Studenten & Anzahl & $\begin{array}{l}\text { Text- } \\
\text { verarbeitung }\end{array}$ & Internet & Email & Programmierung & $\begin{array}{l}\text { eigener PC } \\
\text { zu Hause }\end{array}$ \\
\hline Gesamtkollektiv & 69 & $\begin{array}{c}58 \\
(84,06 \%)\end{array}$ & $\begin{array}{c}68 \\
(98,55 \%)\end{array}$ & $\begin{array}{c}69 \\
(100 \%)\end{array}$ & $\begin{array}{c}4 \\
(5,80 \%)\end{array}$ & $\begin{array}{c}69 \\
(100 \%)\end{array}$ \\
\hline Männer & 15 & $\begin{array}{c}13 \\
(86,67 \%)\end{array}$ & $\begin{array}{c}14 \\
(93,33 \%)\end{array}$ & $\begin{array}{c}15 \\
(100 \%)\end{array}$ & $\begin{array}{c}1 \\
(6,67 \%)\end{array}$ & $\begin{array}{c}15 \\
(100 \%)\end{array}$ \\
\hline Frauen & 54 & $\begin{array}{c}45 \\
(83,33 \%)\end{array}$ & $\begin{array}{c}54 \\
(100 \%)\end{array}$ & $\begin{array}{c}54 \\
(100 \%)\end{array}$ & $\begin{array}{c}3 \\
(5,56 \%)\end{array}$ & $\begin{array}{c}54 \\
(100 \%)\end{array}$ \\
\hline
\end{tabular}

\subsubsection{Erfahrungen im Bereich der Sonographie}

Von 69 Studenten gaben 55 Studenten $(79,71 \%)$ an, keine Erfahrungen im Bereich der Sonographie zu besitzen. 7 Studenten (10,14\%) hatten zum Studienbeginn im Rahmen des Medizinstudiums an einer eineinhalbstündigen Einführung in die Ultraschalldiagnostik teilgenommen und 7 weitere Studenten $(10,14 \%)$ konnten geringe Erfahrungen vorweisen, die z.B. während einer Famulatur oder dem Zivildienst gesammelt wurden.

Das folgende Diagramm macht deutlich, dass die meisten Studenten vor Studienbeginn keine Sonographieerfahrungen besaßen. 


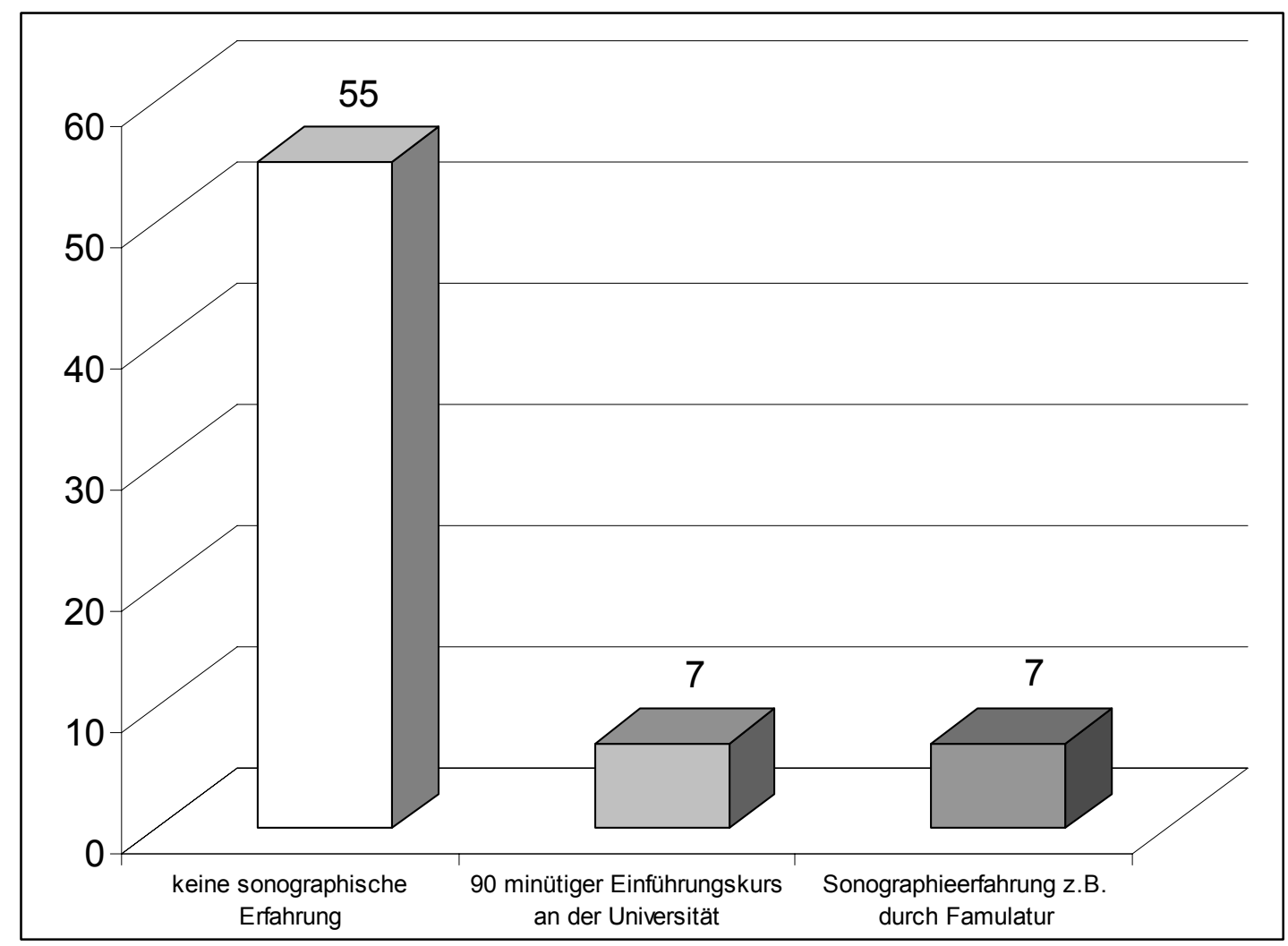

Abbildung 19: Sonographische Erfahrungen der Studenten

\subsection{Charakterisierung des getesteten Kollektivs ( $n=69)$}

Alle 69 Studenten erhielten dieselben Messaufgaben. Zwei Aufgaben wurden zur virtuellen Sonographie und drei Aufgaben zur realen Sonographie gestellt. Die Aufgabe galt als bestanden, wenn der Student in der Lage war, das geforderte Bild auf dem Monitor einzustellen und die korrekte Achse der gewünschten anatomischen Struktur auszumessen. Messwerte, die diesen Anforderungen nicht entsprachen, wurden als ungültig gewertet.

\subsubsection{Virtuelle Sonographie mit dem virtusMED-System}

Die Studenten hatten die Möglichkeit, das virtusMED-System selbständig auszuprobieren. Innen war dabei bewusst kein Zeitlimit gesetzt worden. Die Studenten der Gruppe A (erst virtusMED) probierten das System im Durchschnitt etwa 7 Minuten lang aus, bevor sie den Schallkopf an ihre Kommilitonen weitergaben. Die Studenten der Gruppe B (erst Sono) gaben den Schallkopf etwa zwei Minuten früher weiter. 
Die erste Teilaufgabe (Ausmessen der Tiefe einer definierten Bandscheibe des „virtuellen Patienten“) wurde von 67 der 69 Studenten korrekt durchgeführt. Der Mittelwert aller Studentenwerte liegt bei $39,24 \pm 1,8 \mathrm{~mm}$ und entspricht damit annähernd dem vom Versuchsleiter ermittelten Referenzwert $(39,40 \pm 0,70 \mathrm{~mm})$. Die einzelnen Messergebnisse der jeweiligen Studenten mit Referenzgerade des Versuchsleiters sind in folgender Abbildung dargestellt:

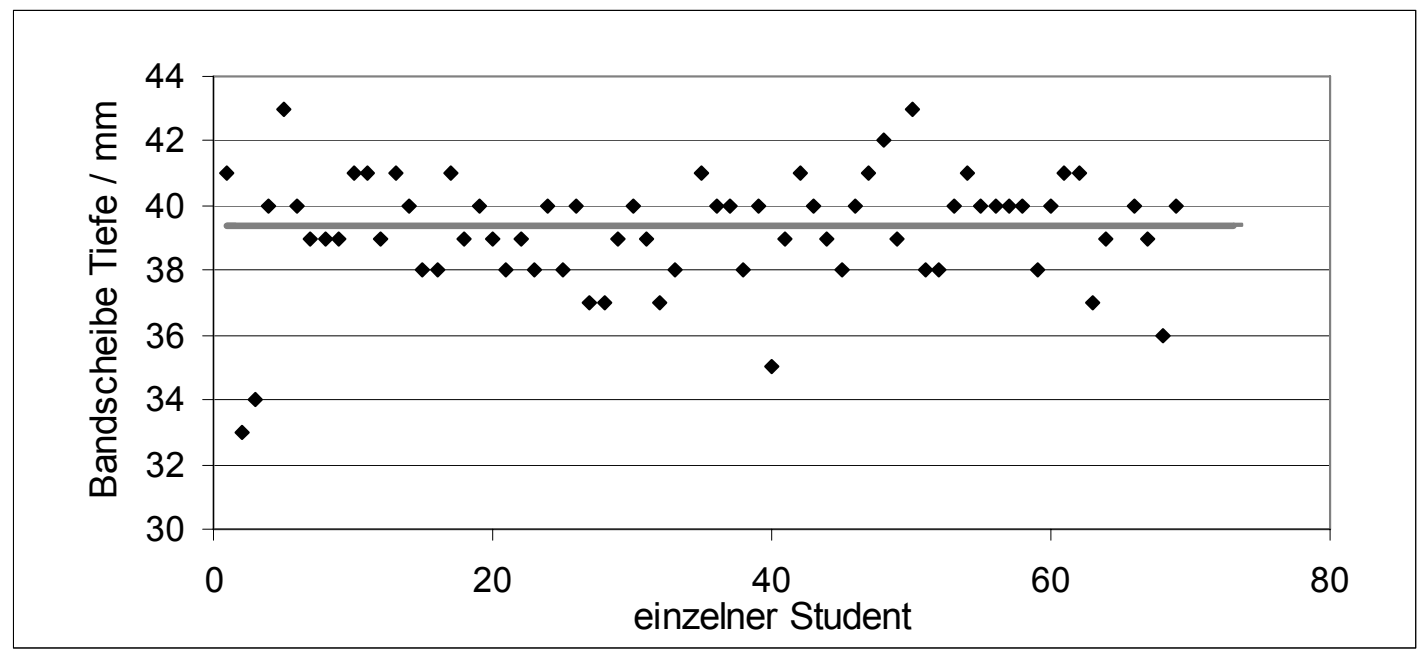

Abbildung 20: Ausmessung der Tiefe einer bestimmten Bandscheibe des virtuellen Patienten. Jeder Punkt steht für einen von einem Studenten ermittelten Wert. Die graue Gerade stellt den Referenzwert dar (vom Versuchsleiter ermittelter Mittelwert).

Die Höhe der Bandscheibe wurde von allen 69 Studenten ordnungsgemäß angegeben. Der Mittelwert aller Studenten liegt bei 10,48 $\pm 0,96 \mathrm{~mm}$, die Referenzgerade bei $10,90 \pm 0,57 \mathrm{~mm}$. Die nachfolgende Graphik zeigt die einzelnen Messergebnisse der Studenten mit Referenzgerade.

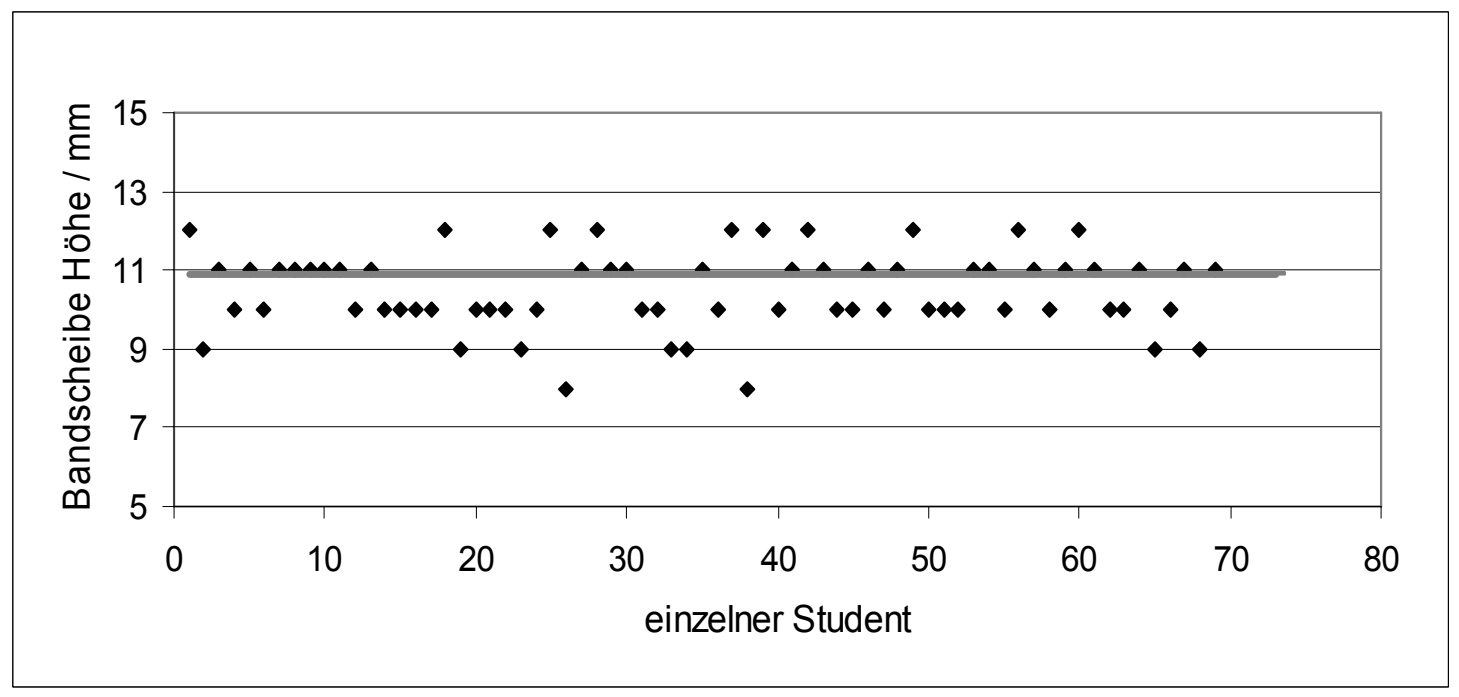

Abbildung 21: Ausmessung der Höhe einer bestimmten Bandscheibe des virtuellen Patienten. Jeder Punkt steht für einen von einem Studenten ermittelten Wert. Die graue Gerade stellt den Referenzwert dar (vom Versuchsleiter ermittelter Mittelwert). 
Die zweite Aufgabe (Ausmessen des Ösophagusdurchmessers des virtuellen Patienten) wurde von 67 der 69 Studenten erfüllt. Der Mittelwert aller gemessenen Ergebnisse liegt bei 23,25 $\pm 2,16 \mathrm{~mm}$, die Referenzgerade bei $23,00 \pm 0,67 \mathrm{~mm}$. Abbildung 22 illustriert die einzelnen Messwerte der Studenten mit Referenzgerade.

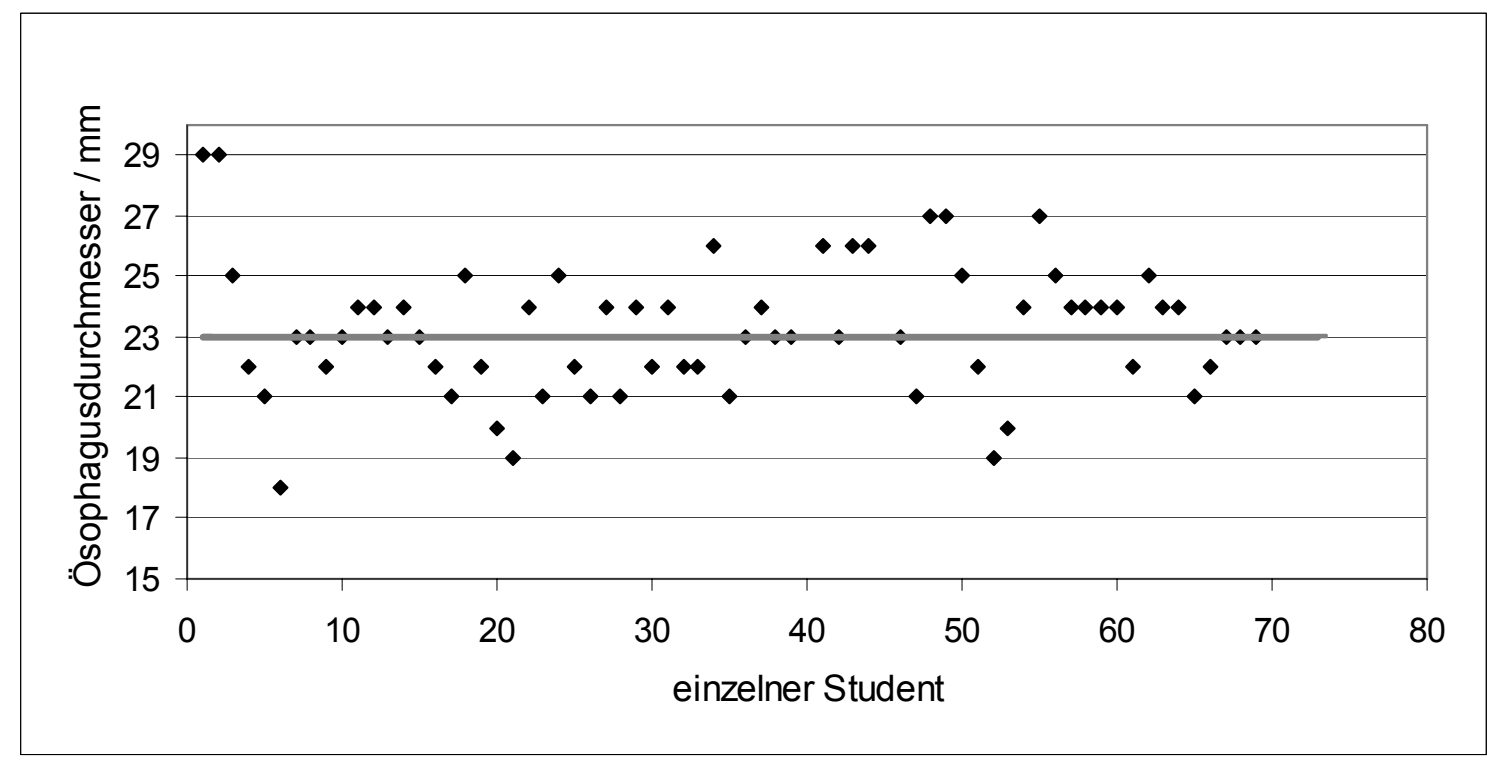

Abbildung 22: Ausmessung des Ösophagusdurchmessers des virtuellen Patienten. Jeder Punkt steht für einen von einem Studenten ermittelten Wert. Die graue Gerade stellt den Referenzwert dar (vom Versuchsleiter ermittelter Mittelwert).

Die Streuung der studentisch ermittelten Messwerte ergibt für die Tiefe der Bandscheibe [43mm-33mm], für die Höhe der Bandscheibe [12mm-8mm] und für den Ösophagusdurchmesser [29mm-18mm].

\subsubsection{Reale Sonographie mit dem Ultraschallgerät SOLINE Elegra von Siemens - Gruppe A (erst virtusMED) vs. Gruppe B (erst Sono)}

Nachfolgende Tabelle stellt die Mittelwerte, die Mediane und die Standardabweichungen dar, die für Testperson ${ }_{1}$ und Testperson $_{2}$ von den Gruppen A und B sowie vom Versuchsleiter zu den jeweils gestellten Aufgaben ermittelt wurden. Es kann von einer Normalverteilung der Messergebnisse ausgegangen werden $\left(p_{\text {gesamt }}=0,1627, p_{\text {GruppeA }}=0,8287, p_{\text {GruppeB }}=0,1888\right)$. Extremwerte finden sich nicht unter den Messwerten der Studenten (siehe Abbildung 27). Die gesamten Messergebnisse der einzelnen Studenten, 
zusätzlich mit Minimum und Maximum, finden sich im Anhang (Tabelle 12, Tabelle 13 und Tabelle 14).

Tabelle 3: Mittelwert, Median und Standardabweichung zu den 3 gestellten Aufgaben für Testperson $_{1}$ und Testperson ${ }_{2}$, jeweils ermittelt von Gruppe A (erst virtusMED), Gruppe B (erst Sonographie) und dem Versuchsleiter

\begin{tabular}{|c|c|c|c|c|c|c|c|c|c|c|}
\hline & \multicolumn{3}{|c|}{ 1. Aufgabe } & \multicolumn{3}{|c|}{ 2. Aufgabe } & \multicolumn{3}{|c|}{ 3. Aufgabe } \\
\hline & & 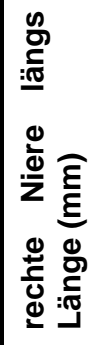 & 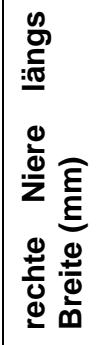 & 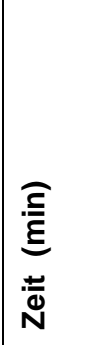 & 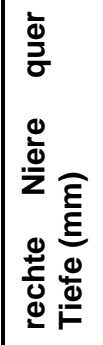 & 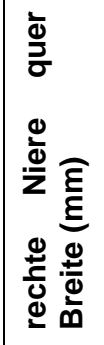 & 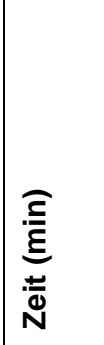 & 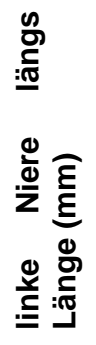 & 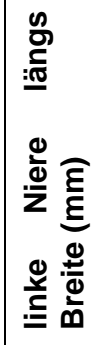 & 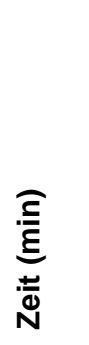 \\
\hline \multicolumn{11}{|c|}{ Testperson $_{1}$} \\
\hline $\begin{array}{l}\text { Gruppe A } \\
\text { (erst } \\
\text { virtusMED) }\end{array}$ & $\begin{array}{l}\text { Mittelwert } \\
\text { Median } \\
\text { Standard- } \\
\text { Abweichung }\end{array}$ & $\begin{array}{l}83,81 \\
84,25 \\
8,82\end{array}$ & $\begin{array}{l}34,62 \\
35,65 \\
3,13\end{array}$ & $\begin{array}{l}00: 25 \\
00: 23 \\
00: 10\end{array}$ & $\begin{array}{l}38,36 \\
38,20 \\
3,29\end{array}$ & $\begin{array}{l}\mathbf{5 3 , 6 4} \\
52,40 \\
7,15\end{array}$ & $\begin{array}{l}00: 19 \\
00: 16 \\
00: 10\end{array}$ & $\begin{array}{r}75,58 \\
72,20 \\
12,55\end{array}$ & $\begin{array}{l}38,18 \\
37,50 \\
6,68\end{array}$ & $\begin{array}{l}\text { 01:18 } \\
01: 02 \\
01: 05\end{array}$ \\
\hline $\begin{array}{l}\text { Gruppe B } \\
\text { (erst Sono) }\end{array}$ & $\begin{array}{l}\text { Mittelwert } \\
\text { Median } \\
\text { Standard- } \\
\text { Abweichung } \\
\end{array}$ & $\begin{array}{l}85,98 \\
84,55 \\
7,29 \\
\end{array}$ & $\begin{array}{l}36,29 \\
36,10 \\
4,20 \\
\end{array}$ & $\begin{array}{l}00: 31 \\
00: 26 \\
00: 18 \\
\end{array}$ & $\begin{array}{l}40,89 \\
39,45 \\
5,18 \\
\end{array}$ & $\begin{array}{l}56,85 \\
56,30 \\
8,36 \\
\end{array}$ & $\begin{array}{l}00: 41 \\
00: 42 \\
\\
00: 22 \\
\end{array}$ & $\begin{array}{r}86,21 \\
86,70 \\
11,05 \\
\end{array}$ & $\begin{array}{l}45,94 \\
44,20 \\
7,66 \\
\end{array}$ & $\begin{array}{l}01: 17 \\
01: 12 \\
00: 59 \\
\end{array}$ \\
\hline $\begin{array}{l}\text { Versuchs- } \\
\text { leiter }\end{array}$ & $\begin{array}{l}\text { Mittelwert } \\
\text { Median } \\
\text { Standard- } \\
\text { Abweichung }\end{array}$ & \begin{tabular}{|l|}
85,89 \\
84,90 \\
3,48 \\
\end{tabular} & $\begin{array}{l}37,20 \\
37,35 \\
1,52 \\
\end{array}$ & $\begin{array}{l}00: 12 \\
00: 11 \\
00: 06\end{array}$ & $\begin{array}{l}45,97 \\
46,05 \\
4,37 \\
\end{array}$ & $\begin{array}{l}\mathbf{5 3 , 5 3} \\
53,40 \\
\\
2,75 \\
\end{array}$ & $\begin{array}{l}00: 11 \\
00: 08 \\
00: 08 \\
\end{array}$ & $\begin{array}{l}94,80 \\
93,70 \\
6,11 \\
\end{array}$ & $\begin{array}{l}45,22 \\
47,90 \\
6,48 \\
\end{array}$ & $\begin{array}{l}01: 12 \\
00: 59 \\
00: 59 \\
\end{array}$ \\
\hline Testperson & & & & & & & & & & \\
\hline $\begin{array}{l}\text { Gruppe A } \\
\text { (erst } \\
\text { virtusMED) }\end{array}$ & $\begin{array}{l}\text { Mittelwert } \\
\text { Median } \\
\text { Standard- } \\
\text { Abweichung }\end{array}$ & $\begin{array}{l}92,77 \\
92,60 \\
8,02 \\
\end{array}$ & $\begin{array}{l}\mathbf{3 8 , 4 6} \\
37,95 \\
\\
2,89 \\
\end{array}$ & $\begin{array}{l}00: 39 \\
00: 22 \\
00: 45 \\
\end{array}$ & $\begin{array}{l}48,64 \\
46,70 \\
8,99 \\
\end{array}$ & $\begin{array}{l}\mathbf{5 0 , 1 4} \\
49,85 \\
7,68 \\
\end{array}$ & $\begin{array}{l}00: 29 \\
00: 23 \\
00: 19 \\
\end{array}$ & $\begin{array}{l}91,92 \\
93,25 \\
9,16 \\
\end{array}$ & $\begin{array}{l}45,55 \\
45,50 \\
5,98 \\
\end{array}$ & $\begin{array}{l}01: 03 \\
00: 42 \\
00: 51\end{array}$ \\
\hline $\begin{array}{l}\text { Bruppe B } \\
\text { (erst Sono) }\end{array}$ & $\begin{array}{l}\text { Mittelwert } \\
\text { Median } \\
\text { Standard- } \\
\text { Abweichung }\end{array}$ & $\begin{array}{l}95,66 \\
96,30 \\
6,11 \\
\end{array}$ & $\begin{array}{l}\mathbf{4 0 , 8 1} \\
39,85 \\
2,97 \\
\end{array}$ & $\begin{array}{l}00: 35 \\
00: 29 \\
00: 21 \\
\end{array}$ & $\begin{array}{l}\mathbf{5 2 , 8 5} \\
53,15 \\
\\
8,99 \\
\end{array}$ & $\begin{array}{l}\mathbf{4 8 , 2 9} \\
47,15 \\
7,15 \\
\end{array}$ & \begin{tabular}{|l|}
$00: 42$ \\
$00: 31$ \\
$00: 39$ \\
\end{tabular} & $\begin{array}{l}95,98 \\
95,90 \\
6,73 \\
\end{array}$ & $\begin{array}{l}46,95 \\
45,70 \\
5,42 \\
\end{array}$ & $\begin{array}{l}00: 57 \\
00: 40 \\
00: 44\end{array}$ \\
\hline $\begin{array}{l}\text { Versuchs- } \\
\text { leiter }\end{array}$ & $\begin{array}{l}\text { Mittelwert } \\
\text { Median } \\
\text { Standard- } \\
\text { Abweichung } \\
\end{array}$ & \begin{tabular}{|l|}
99,51 \\
99,10 \\
3,25 \\
\end{tabular} & $\begin{array}{l}\mathbf{4 2 , 1 6} \\
40,95 \\
3,76 \\
\end{array}$ & $\begin{array}{l}00: 14 \\
00: 11 \\
00: 10 \\
\end{array}$ & \begin{tabular}{|l}
$\mathbf{5 4 , 0 6}$ \\
52,45 \\
5,38 \\
\end{tabular} & \begin{tabular}{|l}
$\mathbf{5 2 , 7 4}$ \\
53,00 \\
5,09 \\
\end{tabular} & \begin{tabular}{|l}
$00: 15$ \\
$00: 15$ \\
$00: 07$ \\
\end{tabular} & $\begin{array}{l}\mathbf{9 8 , 1 5} \\
96,90 \\
5,44 \\
\end{array}$ & \begin{tabular}{|l}
49,27 \\
49,20 \\
3,84 \\
\end{tabular} & $\begin{array}{l}00: 21 \\
00: 16\end{array}$ \\
\hline
\end{tabular}

Es fällt auf, dass beide Gruppen im Durchschnitt geringere Werte messen als der Versuchsleiter. Zusätzlich ist zu beobachten, dass die Messergebnisse der Gruppe A (erst virtusMED) signifikant geringer sind als die der Gruppe B (erst Sonogarphie) (Daten der drei Aufgaben und Unteraufgaben gepoolt: $p=0,0003$ ). Ein Beispiel soll dies verdeutlichen: Der Versuchsleiter ermittelt für Testperson 2

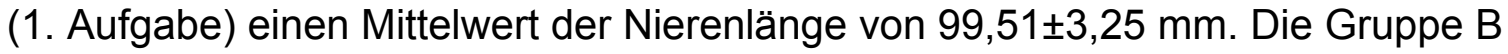
misst eine um 3,85 mm geringere Länge von 95,66 $\pm 6,11 \mathrm{~mm}$. Die Gruppe A 
misst noch einmal 2,89 mm weniger als Gruppe B und im Vergleich zum Versuchsleiter insgesamt $6,74 \mathrm{~mm}$ weniger $(92,77 \pm 8,02 \mathrm{~mm})$.

Betrachtet man die gestellten Aufgaben einzeln, ergibt sich ein signifikanter Messunterschied zwischen Gruppe A und B für Aufgabe $1 \quad(p=0,0231)$ und Aufgabe $3(p=0,0036)$. Bei den Messergebnissen der rechten Niere im Querdurchmesser (Aufgabe 2) ist kein signifikanter Unterschied der beiden Gruppen feststellbar $(p=0,1031)$.

Im Vergleich zu den Messergebnissen der virtuellen Sonographie fallen bei der realen Sonographie höhere Werte für die Standardabweichung auf. Besonders hohe Werte fallen bei Tesperson 1 , 3. Aufgabe auf (Gruppe A 12,55 mm, Gruppe B 11,05 mm). Die Standardabweichung des Versuchsleiters ist im Durchschnitt wesentlich geringer.

Die folgenden zwei Graphiken sollen illustrieren, was zuvor anhand des Beispiels beschrieben wurde. Um die Messergebnisse beider Testpersonen zusammenfassen zu können, wurden die Messergebnisse prozentual umgerechnet. Die meisten Punkte (einzelne Messergebnisse der Studenten) liegen unterhalb der Null-Linie (entspricht dem Mittelwert des Versuchsleiters = Referenzwert), d.h. die Studenten messen eine geringere Länge für die rechte Niere. Die beiden grauen Geraden bilden die Standardabweichung $(3,48 \mathrm{~mm}$ entsprechen 4,05\%) des Versuchsleiters ab, d.h. die Punkte, die sich zwischen diesen beiden Geraden befinden, liegen innerhalb der "normalen“ Schwankungsbreite. In Abbildung 24 liegen mehr Punkte zwischen den beiden grauen Geraden und weniger Punkte unterhalb der $-4,05 \%$ Gerade, als in Abbildung 23. 


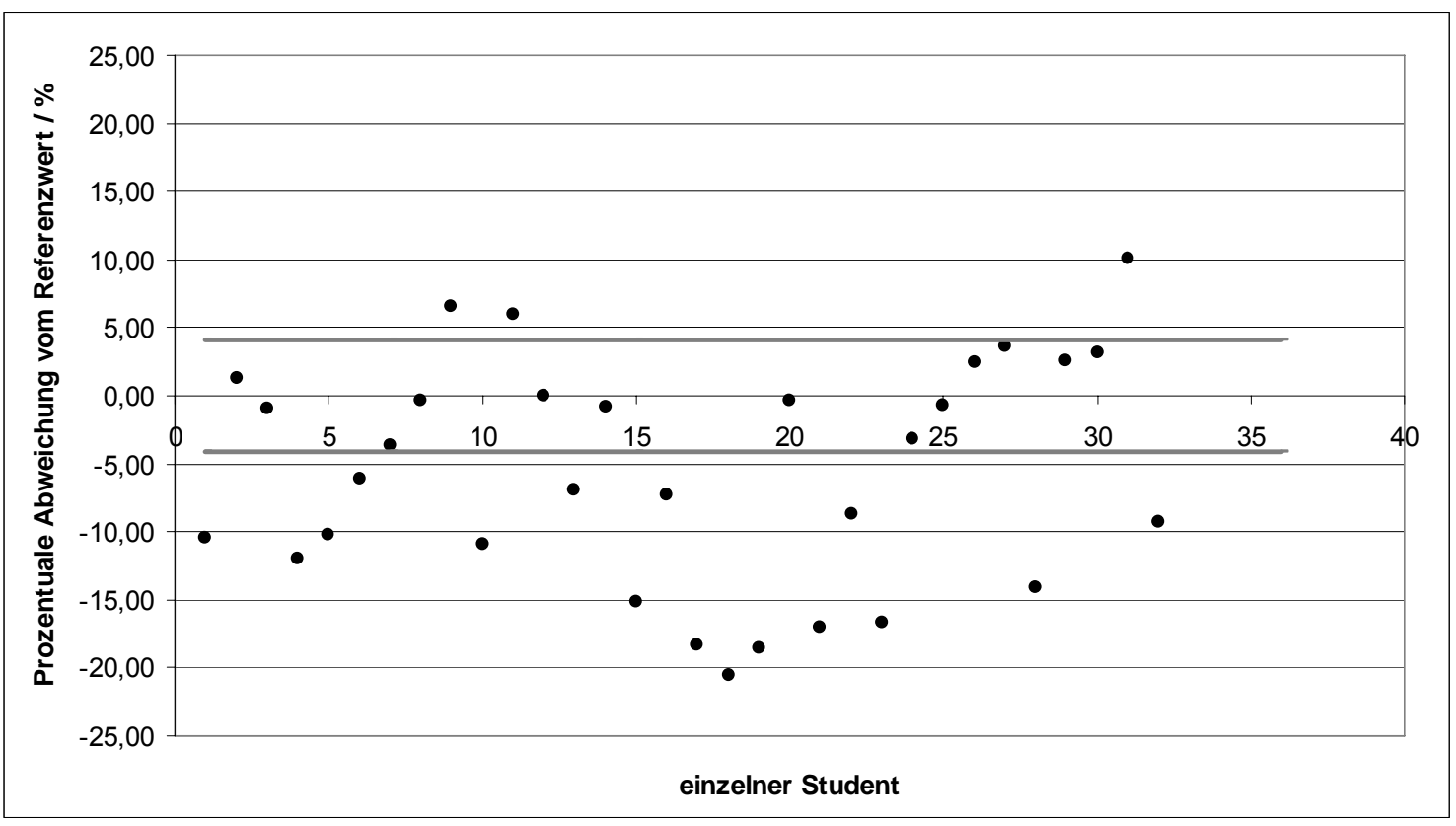

Abbildung 23: Prozentuale Abweichung der von den Studenten ermittelten Messwerte vom Referenzwert - Gruppe A (erst virtusMED) rechte Niere Länge - ; die Nullinie entspricht dem Mittelwert des Versuchsleiters (Referenzwert), die beiden grauen Geraden entsprechen der Standardabweichung $(4,05 \%$ entsprechen $3,48 \mathrm{~mm})$

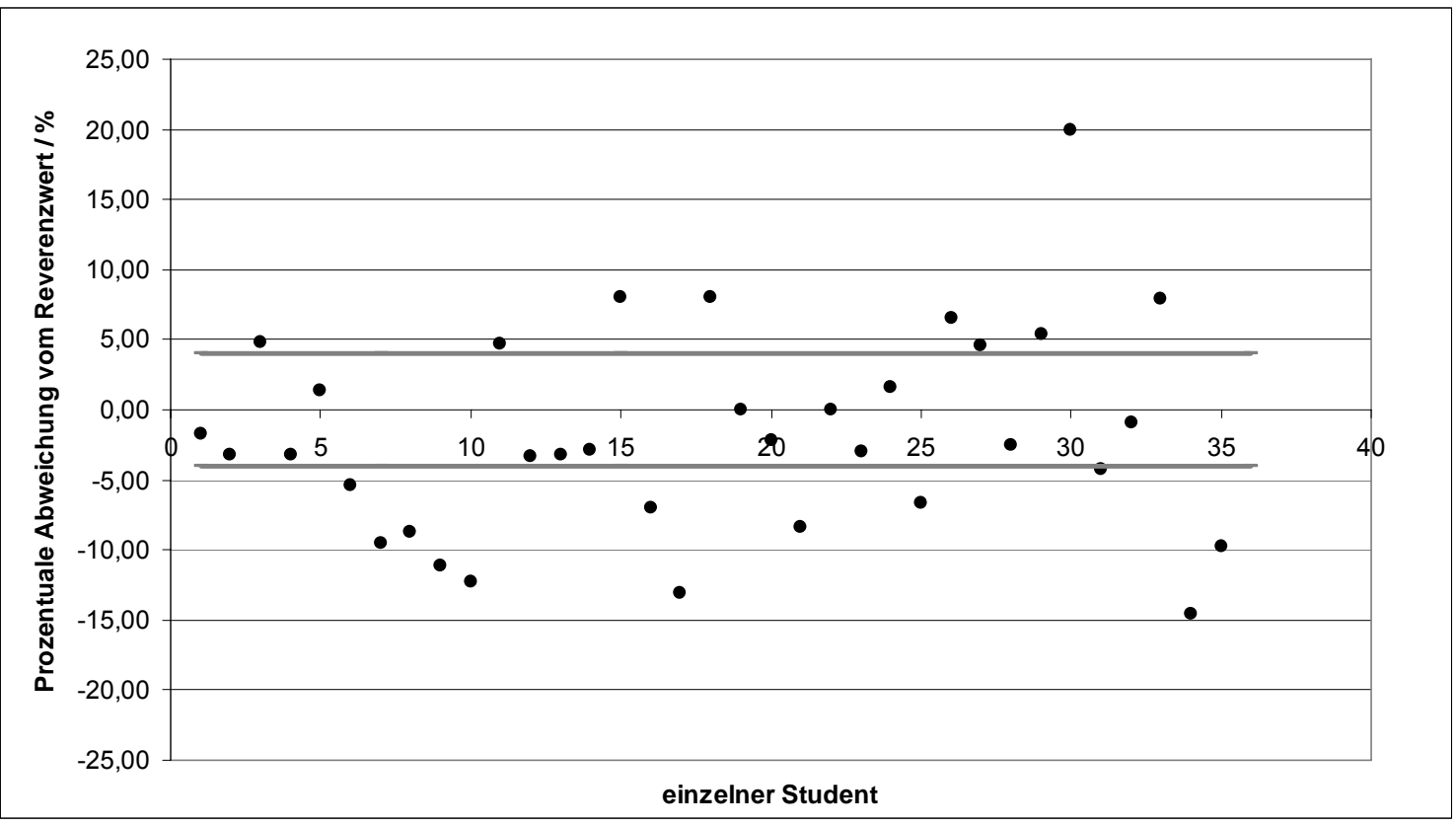

Abbildung 24: Prozentuale Abweichung der von den Studenten ermittelten Messwerte vom Referenzwert - Gruppe B (erst Sonographie) rechte Niere Länge - ; die Nullinie entspricht dem Mittelwert des Versuchsleiters (Referenzwert), die beiden grauen Geraden entsprechen der Standardabweichung (4,05\% entsprechen 3,48 $\mathrm{mm})$

Die beiden folgenden Abbildungen komplettieren die erste sonographisch gestellte Aufgabe, indem die einzelnen Messergebnisse der Nierenbreite dargestellt werden. In Abbildung 25 finden sich keine Werte oberhalb der 
$+8,92 \%$ - Grenze (das entspricht 3,76 mm), dafür umso mehr Werte unterhalb der - 8,92\% - Grenze. In Abbildung 26 liegen wesentlich mehr Punkte innerhalb der vom Versuchsleiter ermittelten Standardabweichung und somit auch näher am Referenzwert, als in Abbildung 25.

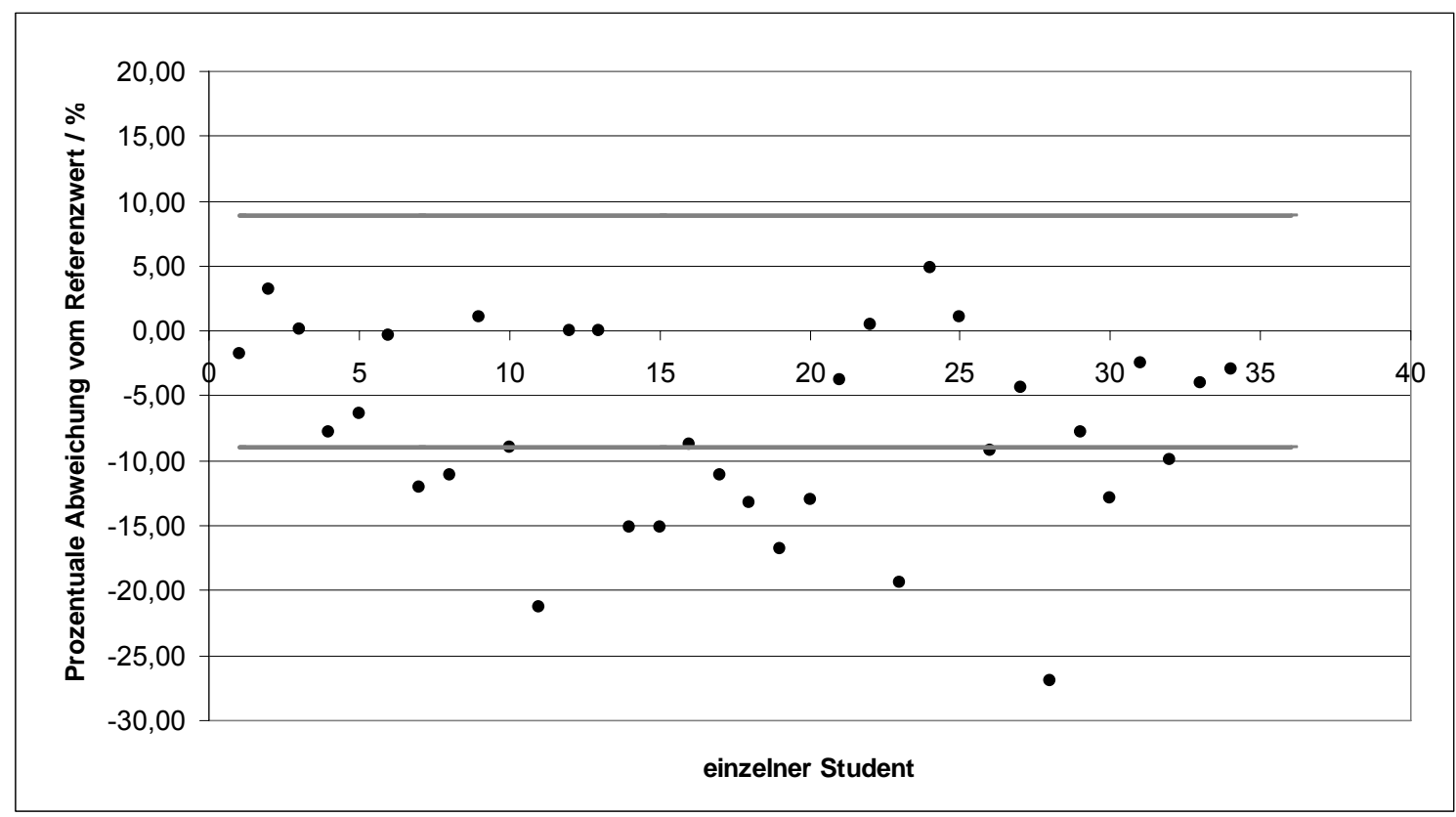

Abbildung 25: Prozentuale Abweichung der von den Studenten ermittelten Messwerte vom Referenzwert - Gruppe A (erst virtusMED) rechte Niere Breite - ; die Nulllinie entspricht dem Mittelwert des Versuchsleiters (Referenzwert), die beiden grauen Geraden entsprechen der Standardabweichung $(8,92 \%$ entsprechen $3,76 \mathrm{~mm})$

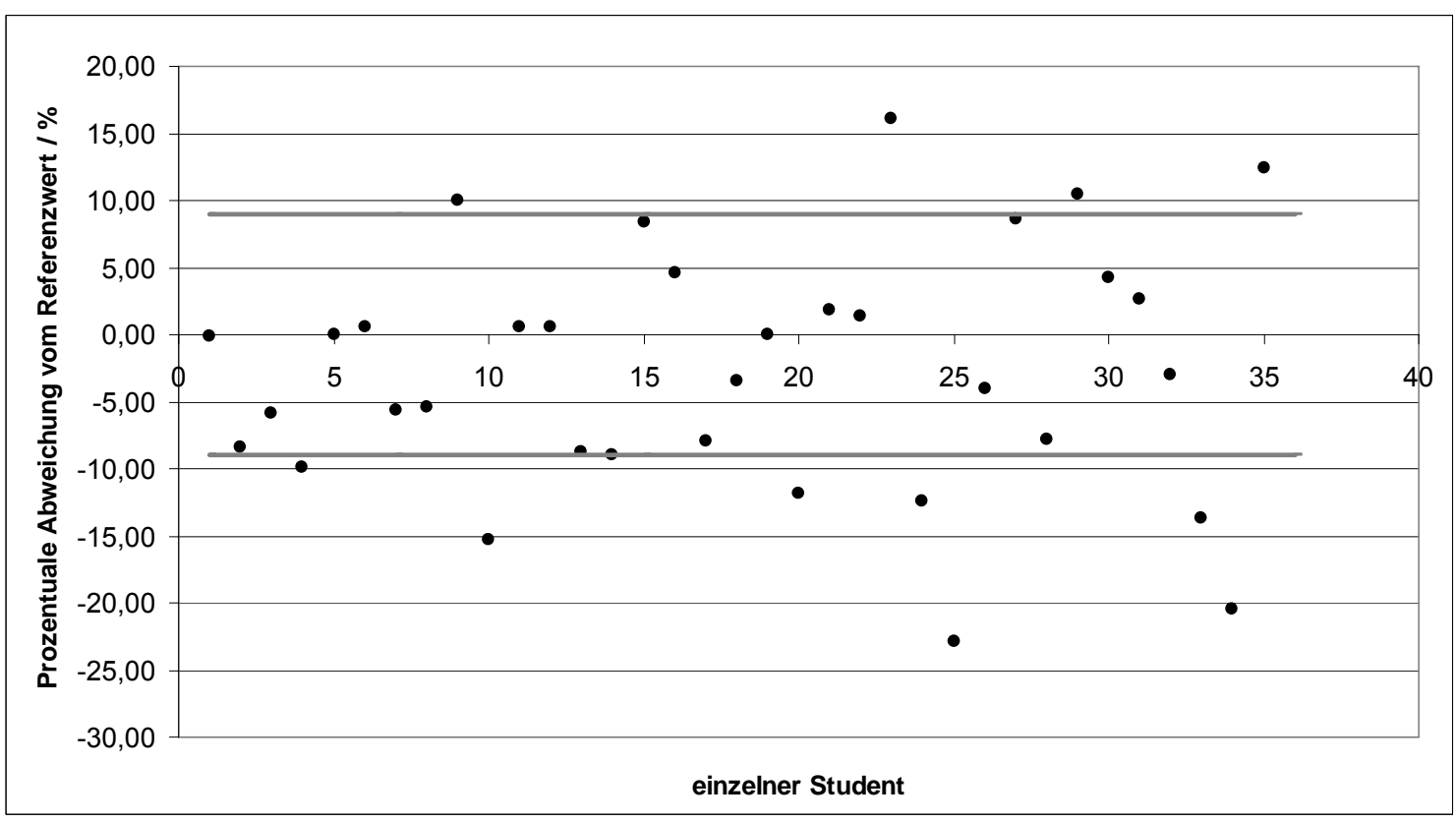

Abbildung 26: Prozentuale Abweichung der von den Studenten ermittelten Messwerte vom Referenzwert - Gruppe B (erst Sonographie) rechte Niere Breite - ; die Nullinie entspricht dem Mittelwert des Versuchsleiters (Referenzwert), die beiden grauen Geraden entsprechen der Standardabweichung $(8,92 \%$ entsprechen $3,76 \mathrm{~mm})$ 
Nachfolgender Box-Plot zeigt, dass beide Gruppen die Messwerte des Versuchsleiters unterschätzen. Zusätzlich veranschaulicht er, dass Gruppe A (erst virtusMED) hoch signifikant kleinere Werte als Gruppe B (erst Sono) misst $(p=0,0003)$.

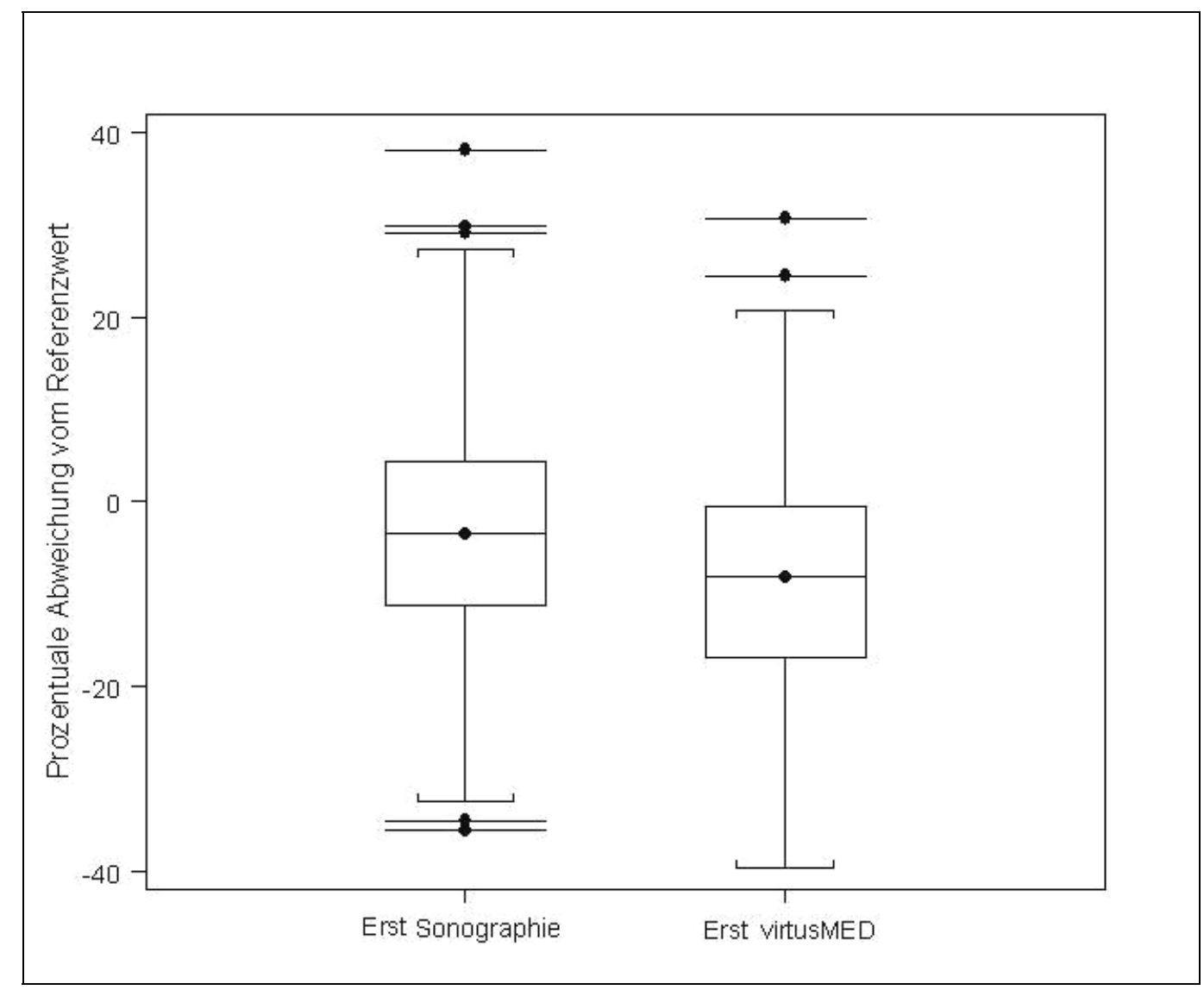

Abbildung 27: Box-Plot: Prozentuale Abweichung der von den Studenten ermittelten Messwerte vom Referenzwert (= Mittelwert des Versuchsleiters) (gepoolte Daten)

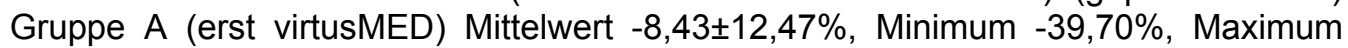
$30,63 \%$, Gruppe B (erst Sonographie) Mittelwert $-3,44 \pm 12,37 \%$, Minimum $-35,63 \%$, Maximum 38,05\%

\subsubsection{Genauigkeit der Messergebnisse in Abhängigkeit von der Zeit}

Es wurde die Zeit gestoppt, die die Studenten benötigten bis sie das optimale Ultraschallbild eingestellt hatten, auf dem die Nierengröße einer Testperson auszumessen war. Die Studenten, die ein virtusMED Vortraining erhalten hatten, waren nicht schneller oder langsamer, als die Studenten ohne Vortraining (t-Test, $\mathrm{p}=0,2414)$.

Die Zeit, die sich die Studenten zum Einstellen des Bildes nahmen, zeigte keinen Einfluss auf die Genauigkeit der Messung $(p=0,5598)$, d.h. ein Student der sich 
viel Zeit zum Aufsuchen der Niere nahm, hat nicht genauer oder ungenauer gemessen, als ein Student, der die Niere in einer kürzeren Zeit einstellen konnte. Die einzelnen Zeiten $z u$ den entsprechenden Aufgaben sowie die Durchschnittszeiten finden sich im Anhang.

\subsubsection{Genauigkeit der Messergebnisse in Abhängigkeit von den Sonographievorkenntnissen}

55 der 69 Studenten $(79,71 \%)$ gaben an, zum Zeitpunkt der Studie keine Vorkenntnisse im Bereich der Sonographie zu besitzen. Geringe Kenntnisse, die beispielsweise durch Famulatur oder Zivildienst erworben wurden, gaben 7 Studenten (10,14\%) an. Weitere 7 Studenten (10,14\%) hatten ihre sonographischen Vorkenntnisse in einem eineinhalbstündigen Kurs im 1. klinischen Semester erworben.

Die unterschiedlichen Vorkenntnisse im Bereich der Sonographie zeigten keinen Einfluss auf die Genauigkeit der Messergebnisse $(p=0,8289)$.

\subsubsection{Genauigkeit der Messergebnisse in Abhängigkeit vom Geschlecht}

Die Studentinnen haben im Vergleich zu den Studenten im Durchschnitt gleichermaßen genau bzw. ungenau gemessen. Die Unterschiede zwischen den Geschlechtern waren nicht signifikant $(p=0,7667)$. Das Geschlecht spielt folglich keine Rolle für die Messgenauigkeit.

\subsubsection{Genauigkeit der Messergebnisse in Abhängigkeit von der Semesteranzahl}

Für die vorliegende Studie wurden vorzugsweise Studenten des ersten klinischen Semesters ausgesucht (57 der 69 Studenten (82,61\%)). 7 Studenten $(10,14 \%)$ stammten aus dem zweiten klinischen Semester und 5 Studenten $(7,25 \%)$ aus dem dritten klinischen Semester.

Die Semesteranzahl zeigte keinen Einfluss auf die Messgenauigkeit der einzelnen Studenten $(p=0,5418)$. 


\subsection{Charakterisierung des befragten Kollektivs $(n=69)$}

Die 69 Studenten, die an dem zweistündigen Ultraschallkurs teilnahmen, erhielten am Ende des Kurses einen Fragebogen, der selbständig auszufüllen war. Im Folgenden werden die Antworten des Fragebogens analysiert.

\subsection{Fragebogenauswertung}

\subsubsection{Multiple-choice-Fragen zur Evaluation des virtusMED-Systems in der sonographischen Lehre sowie zum Gebrauch des virtusMED- Systems}

Den Gebrauch der Untersuchungssonde (Analogie: Ultraschallsonde) und den Einsatz des Styropormodells finden 54 der 69 Studenten (78,26\%) im Hinblick auf die Sonographie als sehr hilfreich. Die dreidimensionale Darstellung auf dem einen Bildschirm, zusätzlich zur zweidimensionalen Ansicht auf dem anderen Bildschirm, wird von über 49 Studenten $(71,01 \%)$ als sehr hilfreich gewertet. Mehr als zwei Drittel der Studenten glauben, dass sie sich durch die Nutzung des virtusMED-Systems bei der Sonographie deutlich besser orientieren können. Die Messfunktion empfinden 36 Studenten (54,55\%) im Hinblick auf die Sonographie als sehr hilfreich. Nur fünfmal wurde das virtusMED-System als wenig hilfreich erachtet.

Die genaue Verteilung (Anzahl der Studenten sowie der prozentuale Anteil) der Antworten auf die jeweiligen Fragen ist in Tabelle 4 dargestellt. 
Tabelle 4: Multiple-choice-Fragen zur Evaluation des virtusMED-Systems in der sonographischen Lehre

\begin{tabular}{|c|c|c|c|c|c|c|}
\hline \multirow[b]{2}{*}{$\begin{array}{l}\text { 1. Finden Sie die } \\
\text { dreidimensionale Darstellung } \\
\text { in der 3D-Ansicht zusätzlich } \\
\text { zur zweidimensionalen Ansicht } \\
\text { der Ultraschallebene hilfreich? } \\
\text { (beantwortet von } 69 \\
\text { Studenten) }\end{array}$} & \multicolumn{2}{|c|}{ auf jeden Fall } & \multirow{2}{*}{$\begin{array}{l}\text { Neutral } \\
\\
\\
4 \\
(5,80 \%)\end{array}$} & \multicolumn{2}{|c|}{ gar nicht } & \multirow{2}{*}{$\begin{array}{l}\text { keine Ein- } \\
\text { schätzung } \\
\\
-\end{array}$} \\
\hline & $\begin{array}{l}49 \\
(71,01 \%)\end{array}$ & \begin{tabular}{|l|}
15 \\
$(21,74 \%)$
\end{tabular} & & $1(1,45 \%)$ & - & \\
\hline $\begin{array}{l}\text { 2. Finden Sie den Gebrauch } \\
\text { der Sonde und des } \\
\text { Styropormodells hilfreich im } \\
\text { Hinblick auf die Sonographie? } \\
\text { (beantwortet von } 69 \\
\text { Studenten) }\end{array}$ & $\begin{array}{l}54 \\
(78,26 \%)\end{array}$ & \begin{tabular}{|l}
12 \\
$(17,39 \%)$
\end{tabular} & $\begin{array}{l}2 \\
(2,90 \%)\end{array}$ & $\begin{array}{l}1 \\
(1,45 \%)\end{array}$ & - & - \\
\hline $\begin{array}{l}\text { 3. Finden Sie die Messfunktion } \\
\text { im Hinblick auf die } \\
\text { Sonographie hilfreich? } \\
\text { (beantwortet von } 66 \\
\text { Studenten) }\end{array}$ & $\begin{array}{l}36 \\
(54,55 \%)\end{array}$ & $\begin{array}{l}22 \\
(33,33 \%)\end{array}$ & $\begin{array}{l}6 \\
(9,09 \%)\end{array}$ & $\begin{array}{l}2 \\
(3,03 \%)\end{array}$ & - & - \\
\hline $\begin{array}{l}\text { 4. Glauben Sie, dass Sie sich } \\
\text { durch die Nutzung des } \\
\text { virtusMED-Systems besser bei } \\
\text { der Sonographie orientieren } \\
\text { können? (beantwortet von } 68 \\
\text { Studenten) }\end{array}$ & $\begin{array}{l}46 \\
(67,65 \%)\end{array}$ & \begin{tabular}{|l|}
18 \\
$(26,47 \%)$
\end{tabular} & $\begin{array}{l}4 \\
(5,88 \%)\end{array}$ & - & - & - \\
\hline
\end{tabular}

Tabelle 5 verdeutlicht, dass 45 Studenten $(65,22 \%)$ das virtusMED-System auf jeden Fall auch außerhalb eines Kurses selbständig nutzen würden. Ein Drittel der Studenten antwortet auf die Frage nach der selbständigen Nutzung mit ,ja vielleicht“. Nur 2 Studenten (2,90\%) können sich eher nicht vorstellen, das virtusMED-System auch außerhalb eines Kurses zu verwenden.

Tabelle 5: Multiple-choice-Fragen zur Evaluation des virtusMED-Systems in der sonographischen Lehre

\begin{tabular}{|l|l|l|l|l|l|}
\hline & $\begin{array}{l}\text { ja auf } \\
\text { jeden Fall }\end{array}$ & $\begin{array}{l}\text { ja } \\
\text { vielleicht }\end{array}$ & $\begin{array}{l}\text { nein eher } \\
\text { nicht }\end{array}$ & $\begin{array}{l}\text { nein auf } \\
\text { keinen } \\
\text { Fall }\end{array}$ & $\begin{array}{l}\text { keine } \\
\text { Einschätzung }\end{array}$ \\
\hline $\begin{array}{l}\text { 5. Können Sie sich vorstellen } \\
\text { das virtusMED-System auch } \\
\text { außerhalb eines Kurses } \\
\text { selbständig zu nutzen? } \\
\text { (beantwortet von 69 } \\
\begin{array}{l}\text { Studenten) } \\
\text { neanten }\end{array}\end{array}$ & $\begin{array}{l}45 \\
(65,22 \%)\end{array}$ & $\begin{array}{l}22 \\
(31,88 \%)\end{array}$ & $\begin{array}{l}2 \\
(2,90 \%)\end{array}$ & - & - \\
\hline
\end{tabular}


Mit der Bedienung des virtusMED-Systems kamen 60 Studenten (86,95\%) sehr gut oder gut zurecht. Die graphische Oberfläche bewerten 65 Studenten $(94,20 \%)$ mit gut bis sehr gut (Tabelle 6).

Tabelle 6: Multiple-choice-Fragen zum Gebrauch des virtusMED-Systems. Die erste Zahl bezieht sich auf die Anzahl der Studenten, die zweite Zahl in Klammern ist der prozentuale Anteil.

\begin{tabular}{|l|l|l|l|l|l|l|}
\hline & sehr gut & & & & & $\begin{array}{l}\text { un- } \\
\text { genügend } \\
6\end{array}$ \\
\hline $\begin{array}{l}\text { 1. Wie gut sind Sie mit der } \\
\text { Bedienung des virtusMED- } \\
\text { Systems zurechtgekommen? } \\
\text { (beantwortet von 69 } \\
\text { Studenten) }\end{array}$ & $\begin{array}{l}23 \\
(33,33 \%)\end{array}$ & $\begin{array}{l}37 \\
(53,62 \%)\end{array}$ & $\begin{array}{l}8 \\
(11,59 \%)\end{array}$ & $\begin{array}{l}1 \\
(1,45 \%)\end{array}$ & - & - \\
\hline $\begin{array}{l}\text { 2. Wie gut gefällt Ihnen die } \\
\text { graphische Oberfläche des } \\
\text { virtusMED-Systems? } \\
\text { (beantwortet von 69 } \\
\text { Studenten) }\end{array}$ & $\begin{array}{l}21 \\
(30,43 \%)\end{array}$ & $\begin{array}{l}44 \\
(63,77 \%)\end{array}$ & $\begin{array}{l}4 \\
(5,80 \%)\end{array}$ & - & - & - \\
\hline
\end{tabular}

\subsubsection{Offene Fragen zur Evaluation des virtusMED-Systems in der sonographischen Lehre sowie zum Gebrauch des virtusMED- Systems}

Neben den Multiple-choice-Fragen enthielt der Fragebogen offene Fragen zur Evaluation des virtusMED-Systems in der sonographischen Lehre sowie zum Gebrauch des virtusMED-Systems. Diese offenen Fragen ließen den Studenten die Möglichkeit in eigenen Worten zusammenzufassen, was ihnen z.B. am virtusMED-System gefallen bzw. nicht gefallen hat. Die meisten Studenten beantworteten die gestellten Fragen.

Zur besseren Übersicht sind die Fragen und eine Auswahl der Antworten (alle Antworten siehe Tabelle 10) in folgender Tabelle zusammengestellt: 
Tabelle 7: offene Fragen zur Evaluation des virtusMED-Systems in der sonographischen Lehre (eine Auswahl, alle Aussagen siehe Tabelle 10)

\begin{tabular}{|c|c|}
\hline $\begin{array}{l}\text { 1. Meinen Sie, der Einsatz des virtusMED- } \\
\text { Systems ist eine nützliche Ergänzung zur } \\
\text { herkömmlichen Ausbildung? } \\
\text { (von } 69 \text { befragten Studenten bejahten } \\
\text { diese Antwort } 68 \text { Studenten) }\end{array}$ & 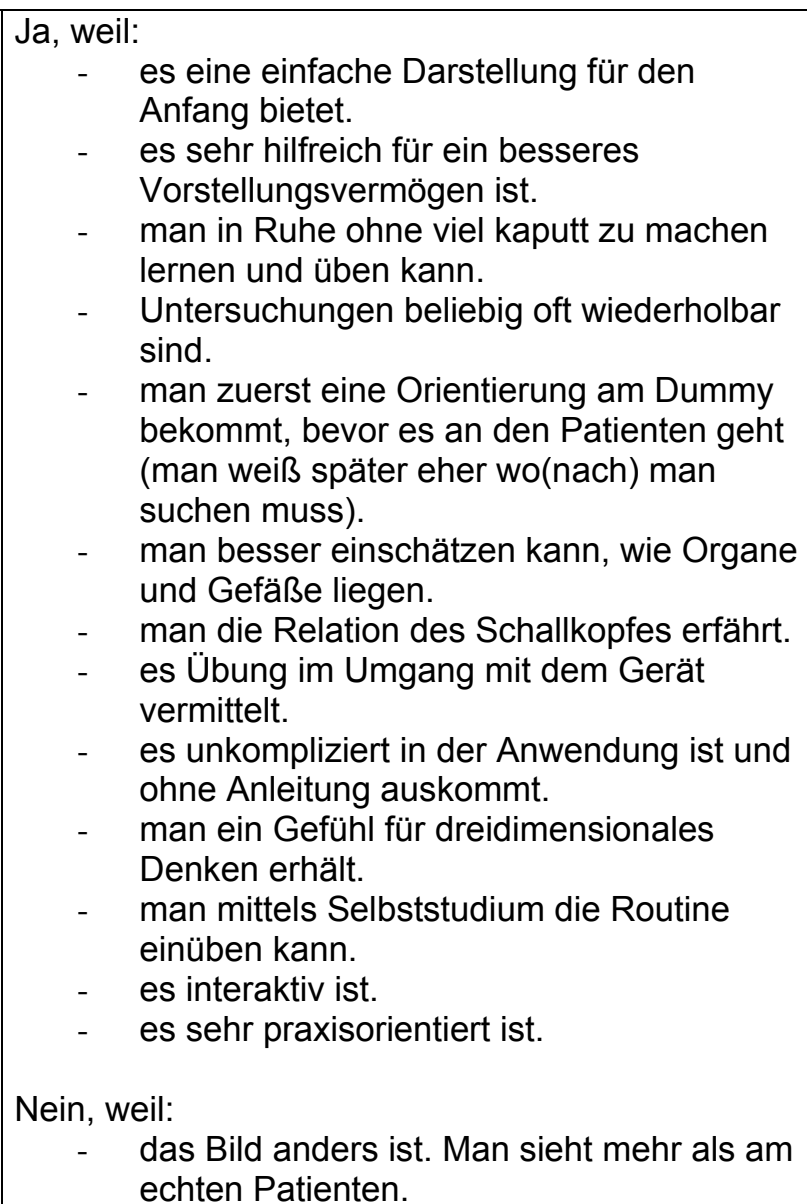 \\
\hline $\begin{array}{l}\text { 2. Was hat Ihnen am virtusMED-System } \\
\text { gut gefallen? }\end{array}$ & $\begin{array}{ll} & \text { die klare Darstellung der Organe und } \\
& \text { daher gute Orientierung für den Anfang } \\
\text { - } & \text { gute Qualität der Bilder, Technik an sich } \\
\text { - } & \text { Anschaulichkeit } \\
\text { - } & \text { Einführung in die Ansichten / Ebenen / } \\
& \text { Strukturen } \\
\text { - } & \text { realistische Handhabung } \\
\text { - } & \text { „geduldiger Patient“, Zeit am Patienten } \\
& \text { kann so effektiv genutzt werden } \\
\text { - } & \text { jederzeit nutzbar, im Gegensatz zum } \\
& \text { - "echten“ Patienten } \\
\text { - } & \text { die einfache Bedienung }\end{array}$ \\
\hline $\begin{array}{l}\text { 3. Was gefällt Ihnen am virtusMED-System } \\
\text { nicht? }\end{array}$ & $\begin{array}{ll}- & \text { "Quietschen“ des Styropormodells, Torso } \\
\text { - } & \text { rechs stabiler liegen } \\
& \text { Ultraschallbild } \\
\text { - } & \text { zu scharf und genau für Sono-Imitation } \\
\text { (trotzdem sinnvoll für Einstieg) } \\
\text { - } \quad \begin{array}{l}\text { es ist keine weibliche Person verfügbar } \\
\text { und keine schwanger Frau }\end{array} \\
\text { - } \quad \text { Animation (pulsierende Gefäße, } \\
\text { Atemverschieblichkeit) } \\
\text { - } \quad \text { keine pathologischen Strukturen sichtbar } \\
\text { - } \quad \begin{array}{l}\text { nicht vergleichbar mit Ultraschallbild, der } \\
\text { direkte Vergleich fehlt }\end{array} \\
\text { - } \quad \begin{array}{l}\text { Helligkeit und Schatten sind nicht immer } \\
\text { repräsentativ }\end{array}\end{array}$ \\
\hline
\end{tabular}


Auf der einen Seite wird die gute Bildqualität und das hohe Auflösungsvermögen des virtusMED-Systems gelobt, auf der anderen Seite bemängeln einige Studenten das Fehlen realer Artefakte, das Ausbleiben von Pulsschlägen oder von Atemverschieblichkeit der Organe sowie pathologischer Befunde.

In der folgenden Tabelle ist eine Auswahl der Antworten (alle Antworten siehe Tabelle 11) auf folgende Fragen zu finden:

- Was würden Sie am virtusMED-System verändern wollen?

- Welche Funktion am virtusMED-System fanden Sie besonders hilfreich?

- Was fehlt Ihnen am virtusMED-System im Vergleich zu den herkömmlichen Ausbildungsmöglichkeiten zum Erlangen der sonographischen Untersuchungstechnik?

Tabelle 8: Offene Fragen zum Gebrauch des virtusMED-Systems (eine Auswahl, alle Aussagen siehe Tabelle 11)

\begin{tabular}{|c|c|}
\hline $\begin{array}{l}\text { 1. Was würden Sie am virtusMED-System } \\
\text { verändern wollen? }\end{array}$ & $\begin{array}{ll}- & \text { anderes Material für Modell } \\
\text { - } & \text { mehr Bezeichnungen / Beschriftungen } \\
\text { - } & \text { Oberfläche „schwammiger“ realitätsnäher } \\
\text { - } & \text { Möglichkeit zwischen fotografischen und } \\
& \text { realen Ultraschallbildern zu wechseln; } \\
& \text { Umschalten von guter Differenzierbarkeit } \\
& \text { auf sonographische Darstellung } \\
\text { - } & \text { reale Artefakte (Schallschatten etc.) } \\
- & \text { weiblicher Patient mit Uterus und Ovar } \\
- & \text { pathologische Veränderungen } \\
- & \text { Es wäre super, wenn man die Pulsschläge } \\
& \text { auch sehen könnte, oder zumindest auf } \\
& \text { Knopfdruck einen kurzen Clip } \\
\text { - } & \text { Prüfungen simulieren (z.B. Abmessen von } \\
& \text { Organen) }\end{array}$ \\
\hline $\begin{array}{l}\text { 2. Welche Funktion am virtusMED-System } \\
\text { fanden Sie besonders hilfreich? }\end{array}$ & $\begin{array}{ll}\text { - } & \text { Benennung der Organe } \\
\text { - } & \text { Schallkopfposition (3D-Ansicht) - bessere } \\
& \text { Orientierungsmöglichkeit durch zwei } \\
& \text { Monitore } \\
\text { - } & \text { Schallkopfbedienung } \\
\text { - } & \text { Zuschalten der Organe auf dem 3D-Modell } \\
\text { - } & \text { Messfunktion } \\
- & \text { Patient drehbar } \\
- & \text { Freeze-Funktion } \\
- & \text { rote und grüne Line zur Orientierung } \\
- & \text { keine Schattierungen } \\
- & \text { CT-Qualität, auch tiefer liegender Organe } \\
& \text { erkennbar }\end{array}$ \\
\hline $\begin{array}{l}\text { 3. Was fehlt Ihnen am virtusMED-System } \\
\text { im Vergleich zu den herkömmlichen } \\
\text { Ausbildungsmöglichkeiten zum erlangen } \\
\text { der sonographischen } \\
\text { Untersuchungstechnik? }\end{array}$ & $\begin{array}{ll}\text { - } & \text { Patientenkontakt } \\
\text { - } & \text { pathologische Befunde } \\
\text { - } & \text { Atemverschieblichkeit, Pulsation von Aorta } \\
& \text { und V. cava } \\
\text { - } & \text { Schallschatten } \\
\text { - } & \text { Es kann das richtige Schallen nicht } \\
& \text { ersetzen, da die Bilder eine zu gute } \\
& \text { Qualität (keine Schatten etc.)haben } \\
\end{array}$ \\
\hline
\end{tabular}


Viele Studenten wünschen sich, dass das virtusMED-System in Zukunft in der sonographischen Ausbildung, aber auch in anderen Bereichen wie der anatomischen Ausbildung eingesetzt wird. Sie finden das Programm praxisorientiert, hilfreich und sinnvoll. Eine Kommentarauswahl der Studenten ist in folgender Tabelle zu aufgelistet:

Tabelle 9: Was Studenten noch sagen wollten (eine Auswahl, alles Aussagen siehe Tabelle 11)

\begin{tabular}{|l|ll|}
\hline & - & Es ist sehr hilfreich und sinnvoll. \\
& - & Ein gutes Programm, um sich an die \\
& Sonographie heran zu tasten und eine \\
& grobe Übersicht der Organe und ihrer \\
1. Was ich noch sagen wollte: & Lage zu bekommen. \\
- & Es ist eine tolle Sache zum besseren \\
& Durchblick durch die Sonographie. \\
- & Genial! Muss Einzug in die Ausbildung \\
& finden! \\
- & Fände es toll, wenn man zur anatomischen \\
& Ausbildung dieses Verfahren einführen \\
& würde. \\
- & Da die Praxis in der Ausbildung viel zu \\
& kurz kommt, sollte es mehr \\
& Lernprogramme dieser Art geben. \\
&
\end{tabular}




\section{Diskussion}

In der medizinischen Ausbildung kommen neben den „klassischen“ Lernformen Lehrbuch, Vorlesung und Unterricht am Krankenbett - zunehmend unterschiedliche multimediale Produkte zum Einsatz (Krüger-Brand 1999, 2002). Diese Produkte ermöglichen es dem Studenten mittels Selbststudium zeit- und oft ortsunabhängig zu arbeiten (Krüger-Brand 2002). Mit Hilfe von Simulationen können komplexe Abläufe oder topographisch-funktionelle Gegebenheiten einfach visualisiert werden (Floto 2002). Eine solche Veranschaulichung könnte auch beim Erlernen der Sonographie helfen, denn vielen Studenten fehlt das dreidimensionale Vorstellungsvermögen, das zum Verstehen der Sonographie notwendig ist.

virtusMED ist eine Lernsoftware, die die Sonographie für Studenten anschaulich und begreifbar machen soll. Ob dieses Computerprogramm hilfreich beim Erlernen der Sonographie ist und ein Einsatz in der medizinischen Ausbildung sinnvoll erscheint, sollte die vorliegende Studie zeigen. Hierzu wurden zum einen die ermittelten Messwerte der Gruppe A, die ein virtusMED Vortraining erhielt, mit denen der Gruppe B (ohne Vortraining) verglichen sowie die Fragebögen analysiert.

Als erstes wird im Folgenden auf die statistische Auswertung der Messergebnisse eingegangen, um anschließend die Auswertung der Fragebögen zu diskutieren.

Die Studenten hatten die Aufgabe am virtuellen Patienten unterschiedliche anatomische Strukturen (Bandscheibe in Tiefe und Höhe sowie Ösophagusdurchmesser) auszumessen. Es sollte festgestellt werden, ob die von innen unter exakt identischen Bedingungen erzielten Messwerte vergleichbar sind. Die geringe Standardabweichung (Bandscheibe Tiefe $=1,8 \mathrm{~mm}$, Bandscheibe Höhe=0,96 mm, Ösophagusdurchmesser=2,16 mm) spricht dafür. Bei der realen Sonographie (unter „normalen“ Bedingungen) ist es, trotz annähernd gleicher Voraussetzungen (gewährleistet durch Testperson ${ }_{1}$ und Testperson ${ }_{2}$ ), nicht möglich eine ähnlich geringe Standardabweichung, wie bei der virtuellen Sonographie, zu erzielen (vgl. höhere Standardabweichung Tabelle 3). Beobachtungen während der Studiendurchführung ließen vermuten, dass es 
den Studenten schwerer fiel die linke Niere, insbesondere der Testperson ${ }_{1}$, auszumessen. Eine besonders hohe Standardabweichung in diesem Fall (linke Niere längs Länge Gruppe $A=12,55 \mathrm{~mm}$, Gruppe $B=11,05 \mathrm{~mm}$ ) könnte diese Beobachtung bestätigen.

Dass Studenten mittels des virtusMED-Systems sehr ähnliche Messergebnisse erzielten, liegt vermutlich an den tatsächlich identischen Bedingungen. Für die reale Sonographie standen zwei Testpersonen zur Verfügung, die den Studenten beispielsweise durch langsames Ein- und Ausatmen oder Luftanhalten das Einstellen von bestimmten Bildern erleichterten. Die Voraussetzungen für die untersuchenden Studenten waren sehr ähnlich, konnten aufgrund physiologischer Gegebenheiten aber niemals so identisch wie bei der virtuellen Sonographie sein.

Aufgrund der geringen sonographischen Erfahrung der Studenten war zu erwarten, dass diese den Referenzwert nicht immer exakt treffen würden. Auffällig ist jedoch, dass beide Gruppen den Referenzwert im Durchschnitt unterschätzten, d.h. sie ermittelten im Durchschnitt eine kürzere Länge für das geforderte Organ (siehe Abbildung 23, Abbildung 24, Abbildung 25, Abbildung 26). Schon während der Studiendurchführung fiel auf, dass Studenten vornehmlich zu kurz als zu lang maßen. Vielleicht wähnten sie sich so auf der „sicheren Seite“.

Statistisch betrachtet erzielte Gruppe A, mit einem virtusMED-Vortraining, signifikant kürzere Messergebnisse als Gruppe $B$ ( $p=0,0003$, gepoolte Daten). Dies wird zusätzlich im Box-Plot (Abbildung 27) und in den Abbildungen Abbildung 23 und Abbildung 26 ersichtlich. Das bedeutet, dass Studenten trotz des virtusMED-Vortrainings ungenauere Messergebnisse erzielten.

Betrachtet man die Messunterschiede von Gruppe A und B für die jeweiligen Aufgaben separat, ist ein signifikanter Unterschied der Messergebnisse der beiden Gruppen für Aufgabe 1 ( $p=0,0231$, rechte Niere längs, Länge und Breite) und 3 ( $p=0,0036$, linke Niere längs, Länge und Breite) zu erkennen. Für Aufgabe 2 ist kein signifikanter Unterschied festzustellen $(p=0,1031)$. D.h., dass die Studenten der Gruppe A und B den Querdurchmesser und die Tiefe der rechten Niere gleich gut bzw. schlecht ermittelten. Da es sich bei dem Querdurchmesser 
einer Niere annähernd um eine kreisrunde Form handelt, verwundert es nicht, dass die Messergebnisse einander derart gleichen. Die Aussagekraft ist in dieser Hinsicht beschränkt.

Eine größere Stichprobenzahl kann die Aussagekraft erhöhen. Aus diesem Grund wurden die gesamten Messergebnisse der drei gestellten Aufgaben für die Varianzanalysen zusammengefasst, so dass sich eine Stichprobenzahl von n=195 für Gruppe $A$ und $n=197$ für Gruppe $B$ ergab. Ob eine größere Stichprobenzahl für die Gruppen $A$ und $B\left(n_{A}=34\right.$ und $\left.n_{B}=35\right)$ ein anderes Ergebnis erbracht hätte, ist fragwürdig.

Dadurch, dass Gruppe A und B vor dem realen Sonographietest selbständig explorieren konnten, gleicht sich das Wissen vor dem Test vermutlich zu sehr, als dass das virtusMED-System einen wirklichen Effekt zeigen könnte. Wäre das eigenständige Explorieren weggelassen worden, hätte die Studie genauer untersucht, ob das System eine Hilfe bei der realen Sonographie ist.

Ein weiterer Erklärungsansatz für die größere Abweichung der von Gruppe A ermittelten Messergebnisse vom Referenzwert könnte die mangelnde Konzentration am Ende des zweistündigen Ultraschallkurses gewesen sein.

Gruppe B erhielt als erstes eine Einführung in die reale Sonographie und konnte somit nach einer Stunde den Test absolvieren. Gruppe A absolvierte erst am Ende der zweiten Stunde den Test. Es wäre denkbar, dass die Studenten zu diesem Zeitpunkt schon etwas erschöpft, unkonzentrierter und nicht mehr im vergleichbaren $\mathrm{Ma}$ aufnahmefähig waren. Das würde für ungenauere Messergebnisse sprechen, allerdings nicht erklären, warum durchschnittlich signifikant kürzere und nicht beispielsweise längere Messergebnisse ermittelt wurden. Längere Messergebnisse wurden vermutlich nicht erzielt, da es unmöglich ist ein Organ größer auszumessen, solange man sich an die Begrenzung hält. Durch abweichende Achseneinstellung und zaghaftes Messen ist es aber durchaus möglich, zu kurz zu messen.

Die Untersuchungsbedingungen bei der virtuellen Sonographie sind für Studenten besonders gut, annähernd identisch und vermutlich einfacher als bei der realen Sonographie. Absolventen eines Simulationstrainings könnten sich dadurch in scheinbarer Sicherheit wähnen (Strauss und Heer 2006), auch unter physiologischen Bedingungen geforderte Organe schnell und einfach zu finden. Die Konfrontation realer Untersuchungsbedingungen mit ungünstigen 
Schallbedingungen kann daher einen Lerneffekt mittels Simulator in Frage stellen (Strauss und Heer 2006). Es könnte sein, dass die Studenten der Gruppe $A$, die ein virtusMED-Vortraining erhielten, zu selbstsicher an die Aufgaben herangingen bzw. sie in ihrer Schwierigkeit unterschätzten und dadurch eventuell weniger konzentriert waren und ungenauer maßen.

Auch wenn der Unterschied zwischen den beiden Gruppen statistisch betrachtet signifikant ist, fällt es schwer, eine eindeutige Aussage bezüglich des Lerneffektes daraus zu ziehen. Es ist schwierig ein objektives Verfahren zur Ermittlung des Lerneffektes zu finden. Es ist denkbar, dass die Aufgabenstellung (Erfassung von Länge, Breite und Tiefe der Nieren) der vorliegenden Studie nicht aussagekräftig genug ist, um eindeutig zu behaupten, dass ein Vortraining mit dem virtusMED-System sinnvoll bzw. sinnlos ist.

Eine Studie (Maul et al. 2004) aus dem Bereich der Gynäkologie zur Überprüfung der Effektivität eines SonoTrainers war ähnlich konzipiert. Das Ergebnis: Die Ärztegruppe mit theoretischer Einführung in die Sonographie mit zusätzlich praktischer Übung am SonoTrainer erzielte sowohl bei der Bestimmung der Scheitelsteißlänge (CRL) als auch der Nackentransparenz (NT, „Nackenfalte") signifikant geringere Messabweichungen vom definierten Standard, als die Vergleichsgruppe, die nur eine theoretische Erklärung erhielt. Daraus wurde geschlussfolgert, dass der verwendete Ultraschallsimulator zur Verbesserung der Untersuchungskompetenz beiträgt, das Training am Patienten jedoch nicht vollständig ersetzen kann (Wüstermann et al. 2002, vgl. Strauss und Heer 2006).

Während die Studenten das optimale Bild zum Ausmessen der gewünschten Struktur einstellten, wurde die Zeit gestoppt. Anschließend wurde überprüft, ob es einen Zusammenhang zwischen benötigter Zeit und Messgenauigkeit gibt. Statistisch betrachtet ließ sich kein signifikanter Unterschied $(p=0,5598)$ feststellen. Die Studenten waren zuvor nicht von der Zeitmessung in Kenntnis gesetzt worden, um sie nicht zusätzlich unter Druck zu setzen. Einige Studenten hätten vermutlich versucht möglichst schnell ein Ergebnis zu erzielen, dass dann jedoch ungenauer hätte werden können. Viele Studenten merkten dennoch, dass die Zeit gemessen wurde, ließen sich meist jedoch nicht aus der Ruhe bringen. Einige Studenten nahmen sich Zeit, um die genaue Einstellung zu 
finden, andere hatten "Glück“ und erhielten gelegentlich „Zufällig“ das gewünschte Bild und benötigten entsprechend weniger Zeit. Aus diesem Grund lässt sich vermutlich kein Zusammenhang zwischen benötigter Zeit und Messgenauigkeit feststellen.

Für die vorliegende Studie wurden bevorzugt Studenten ohne Ultraschallvorkenntnisse ausgewählt. 14 Studenten (20,29\%) hatten (sehr) geringe Vorkenntnisse, die sie durch Famulatur, Zivildienst oder im Rahmen einer studentischen Veranstaltung (einem neunzigminütigen Einführungskurs in die Sonographie) erworben hatten. Ein signifikanter Unterschied zwischen der Messgenauigkeit der Studenten ohne Sonographievorkenntnisse und denen mit geringen Vorkenntnissen ließ sich nicht feststellen $(p=0,8289)$. Dies ist aufgrund der geringen Sonographieerfahrung allerdings nicht verwunderlich.

Auch die Semesteranzahl zeigte keinen Einfluss auf die Messgenauigkeit $(p=0,5418)$. Das liegt vermutlich daran, dass die Studenten in höheren Semestern nicht mehr Sonographiekenntnisse besitzen als die in einem niedrigeren Semester. Umso wichtiger ist es, diese Studenten zu schulen, damit sie bessere Kenntnisse im Umgang mit der Sonographie erlangen. Der Einsatz des virtusMED-Systems könnte an dieser Stelle helfen.

Im Folgenden wird auf die Auswertung des Fragebogens eingegangen.

Die Bedienung des virtusMED-Systems bewerten 60 von 69 Studenten (86,96\%) als gut oder sehr gut. Mit der Schulnote 3 (befriedigend) bewerten das Programm 8 Studenten (11,59\%). Lediglich ein Student (1,45\%) findet die Bedienung ungenügend. Die Software wird augenscheinlich gut angenommen und ist nach Angaben der Studenten hinsichtlich der Bedienung nicht wesentlich verbesserungswürdig. Die graphische Oberfläche wird von 21 Studenten $(30,43 \%)$ mit sehr gut und von 44 Studenten $(63,77 \%)$ als gut bewertet. Auch an dieser Stelle muss das Programm nicht zwangsläufig optimiert werden. Damit ein Computer-Lernprogramm bevorzugt verwendet wird, ist eine einfache, intuitive Bedienung, wie sie das virtusMED-System bietet, notwendig. Unklare Navigation frustriert und schreckt den Benutzer ab (Floto 2002). 
Die verschiedenen Funktionen, die das virtusMED-System bereit hält, werden durchgehend positiv bewertet. Besonders hilfreich finden die Studenten die Benutzung der Untersuchungssonde und das Styropormodell (siehe Tabelle 8). Eine gute Hand-Auge-Koordination ist für eine erfolgreiche Sonographie essenziell. Umso positiver ist es, dass viele Studenten die Handhabung der Sonde in Kombination mit dem Styroportorso als realitätsnah und hilfreich beschreiben. Die Vermutung, dass Studenten der Umgang mit realer Ultraschallsonde und realem Ultraschallgerät nach einem Vortraining mit dem virtusMED-System leichter fallen wird, liegt nahe. Das Quietschen des Styroportorsos wird von einigen als unangenehm angegeben. Aus diesem Grund sollte überlegt werden, das Styropor gegen ein anderes Material zu ersetzten.

Auf jeden Fall als hilfreich wird die dreidimensionale Darstellung des virtuellen Patienten von 49 Studenten $(71,01 \%)$ bewertet. Die Studenten scheinen erst durch diese Ansicht zu verstehen, in welchem Winkel die Schallwellen der Sonde in den Körper gelangen und welche Ebene daraus in der 2D Ansicht (Ultraschallmodus) resultiert. Dies ist vermutlich eine der wesentlichen Funktionen des virtusMED-Systems, die es den Studenten ermöglicht, die Sonographie besser zu begreifen. Mit Worten den Weg der Schallwellen zu beschreiben, ist wesentlich komplizierter, als einen Blick auf die 3D-Ansicht zu werfen.

64 von 68 Studenten $(94,12 \%)$ sind der Ansicht, dass sie sich durch die Nutzung des virtusMED-Systems besser bei der Sonographie orientieren können. Dies bestätigt die zu Beginn der Studie aufgestellte 3. Hypothese. Es verwundert umso mehr, dass die Studenten, die ein virtusMED Vortraining erhielten und sich subjektiv besser bei der Sonographie orientieren konnten, ungenauere Messergebnisse bei der realen Sonographie ermittelten. Eventuell sind diese beiden Ergebnisse aber unanhängig von einander zu betrachten.

Im Sommersemester 2007 hatten 69 Studenten der Georg-August-Universität Göttingen die Möglichkeit an vorliegender Studie und damit einem zweistündigen Ultraschallkurs teilzunehmen. Der Andrang interessierter Studenten an diesem Kurs teilzunehmen war so groß, dass der Nachfrage aus Zeit- und 
Kapazitätsgründen nicht nachgekommen werden konnte. Die Studierenden beklagten die mangelhafte und zu kurze Ausbildung, die das Studium für eine ihnen wichtig erscheinende Diagnostik bietet. Eine in den USA durchgeführte Studie ergab ähnliche Beobachtungen. 67\% der befragten Assistenzärzte gaben an, weder theoretisch noch praktisch in der Sonographie ausgebildet worden zu sein. Bei $90 \%$ dieser Ärzte hätte allerdings Interesse bestanden an einem Ultraschallkurs teilzunehmen (Hoffmann 2003). Hieraus wird ersichtlich, wie wichtig Studenten die Sonographieausbildung bereits während des Studiums ist. Eine weitere Untersuchung (Kratzer et al. 2000) zum Sonographielehrangebot stellte ebenfalls ein großes Interesse am abdominellen Ultraschall fest. Die Bedeutung der Methode Ultraschall wurde von den Studenten für die spätere ärztliche Tätigkeit als extrem hoch eingestuft (Kratzer et al. 2000).

Dass Studenten die umfassende Diagnostik der Sonographie nicht ausreichend während des Studiums lernen können, wird deutlich, wenn man bedenkt, dass ein erfahrener Ultraschalluntersucher nach Angaben der Deutsche Gesellschaft für Ultraschall in der Medizin (DEGUM) erst nach 10 Jahren die oberste Stufe der DEGUM erreicht. Es sollte dennoch versucht werden, den Studenten während des Studiums einen Einblick in die vielfältigen Möglichkeiten und verschiedenen Indikationsstellungen der Sonographie zu geben. Sie sollten sich über die große Aussagekraft dieser ungefährlichen und kostengünstigen Methode bewusst werden, denn schließlich ist es das am häufigsten eingesetzte bildgebende Verfahren in der Medizin. Dass das Interesse an der Sonographie groß ist, zeigt die starke Nachfrage der Studenten, die an dieser Studie teilnehmen wollten. Es wäre folglich eine Überlegung wert, zukünftig ein Wahlfach Sonographie anzubieten. Ein solches Wahlfach könnte die medizinische Ausbildung um einen sehr praxisbezogenen Aspekt, wie es die neue Approbationsordnung (Neue AO 2002) fordert, bereichern.

45 der 69 Studenten (65,22\%) können sich auf jeden Fall vorstellen, dass virtusMED-System auch außerhalb einer universitären Pflichtveranstaltung zu nutzen. Immerhin antworten 22 Studenten (31,88\%) auf die Frage mit ja vielleicht und nur 2 Studenten (2,90\%) können sich eher nicht vorstellen, das virtusMED-System in ihrer Freizeit zu nutzen. Die große Anzahl der Studierenden, die das virtusMED-System auch außerhalb eines Kurses nutzen 
würden, bestätigt die Vermutung, dass das Interesse an der Sonographie groß ist und zeigt außerdem, dass eine hohe Bereitschaft zur Eigeninitiative bzw. zum Selbststudium vorliegt. Viele Studenten wünschen sich neben dem öffentlichen Zugang des virtusMED-Systems eine ähnliche Betreuung, wie im Rahmen der vorliegenden Studie. Diese Betreuung könnte beispielsweise durch einen erfahrenen Studenten realisiert werden.

Einige Studien haben gezeigt (vgl. Arnold und Langkafel 2001, Frey P 1999, Rosendahl 2003), dass sich Studenten durchaus mit Lernprogrammen weiterbilden würden, wenn sie wüssten, was für verschiedene Angebote und Möglichkeiten zur Verfügung stehen. Nach Frey werden ComputerLernprogramme unter anderem gut genutzt, wenn sie in Lehrveranstaltungen eingebunden werden oder der Prüfungsvorbereitung dienen. An der GeorgAugust-Universität Göttingen haben Studenten im Lernstudio der Chirurgie (http://wwwuser.gwdg.de/ chirurg/lernstudio/) die Möglichkeit verschiedene Lernprogramme zu verwenden, um sich damit auf kommende Klausuren vorzubereiten. Da viele Studenten oft nicht über das (multimediale) Angebot an ihrer eigenen Universität informiert sind, ist es hilfreich, wenn Dozenten auf neue Möglichkeiten hinweisen (Frey P 1999).

Neue Medien bekommen in der Medizin zurzeit eine immer größere Bedeutung, bieten jedoch keine Alternative (Floto 2002) zur praktischen, patientennahen Ausbildung, dafür aber eine sinnvolle Ergänzung. Computerprogramme, vergleichbar dem virtusMED-System, unterscheiden kaum mehr zwischen Übung und tatsächlicher Untersuchung, wodurch eine ganzheitliche Lernmethodik geschaffen wird (Teistler 2004). Weitere Beispiele für Simulatoren sind der SimMan ${ }^{\circledR}$ oder Harvey. Diese bieten Studenten Gelegenheit, sich im Intubieren oder Drainagelegen zu üben sowie Herztöne und Herzgeräusche zu differenzieren. Solche Simulationen können auch als Mock-up (reales Teilmodell) bezeichnet werden. Der reale Teil in diesem Modell überwiegt und wird durch einen virtuellen Teil ergänzt. Wir befinden uns sozusagen zwischen Virtualität und Realität bzw. in der sog. erweiterten Realität (Augmented Reality). 
68 der 69 Studenten (98,55\%) sind aus verschiedensten Gründen der Ansicht, dass das virtusMED-System eine nützliche Ergänzung zur herkömmlichen Ausbildung ist (siehe Tabelle 7).

Die gute Bildqualität und die einfache Darstellung helfen den Studenten sehr, sich besser im menschlichen Körper zu orientieren. Die Handhabung der Untersuchungssonde gleicht der der realen Ultraschallsonde, so dass der Umgang damit praxisorientiert und realistisch ist. Viele Studenten haben Probleme damit, das zweidimensionale Ultraschallbild zu deuten. Das virtusMED-System schult nach Angaben der Studenten das dreidimensionale Denken und verbessert das Vorstellungsvermögen. Diese Fähigkeit ist unabdingbar zur Beurteilung von Ultraschallbildern, aber auch zur selbständigen Durchführung einer sonographischen Untersuchung.

Es kommt vor, dass der „richtige“ Patient für eine Untersuchung bzw. den Unterricht am Krankenbett nicht zur Verfügung steht, wenn er benötigt wird, oder unkooperativ ist. Diesem Problem kann Abhilfe geschaffen werden, da der „virtuelle“ Patient jederzeit für eine Untersuchung bereit steht. Der zeit- und ortsunabhängige Zugang ist ein wesentlicher Vorteil, den computergestützte Lernsysteme bieten (Krüger-Brand 2002). Zusätzlich ermöglichen sie ein selbständiges, interaktives Aneignen der Fähigkeiten bzw. des Wissens.

Einige Studenten meinen sich vor dem Patienten zu blamieren, wenn sie eine bestimmte Methodik, beispielsweise die der Sonographie, noch nicht beherrschen. Andere befürchten sogar den Patienten zu verletzen. Der Vorteil klinischer Simulationen ist, die Verbesserung verschiedener Fertigkeiten mit „Hands-on-Erfahrung“, ohne dem Patienten dabei zu schaden (vgl. http://www.hret.org/hret/programs/simulations.html). E-Learning ermöglicht Studenten außerdem in einer ruhigen und sicheren Umgebung angstfrei problemorientiert zu lernen (Docherty et al. 2005). Das virtusMED-System bietet die Möglichkeit, einen geduldigen, virtuellen Patienten in Ruhe beliebig oft zu untersuchen, „ohne, dass man viel kaputt machen kann“ (Studentenzitat siehe Tabelle 10). Außerdem gilt: Der virtuelle Patient beschwert sich nicht (Page 2002)! Mittels Selbststudium kann so eine gewisse Routine eingeübt werden. Der Student fühlt sich dadurch besser für den „Ernstfall“ (Studentenzitat, siehe Tabelle 10) vorbereitet. Außerdem hat er durch vorheriges Training mit dem virtusMED-System die anatomischen Gegebenheiten wiederholt und weiß 
zusätzlich besser, anhand welcher Strukturen er sich orientieren kann. „Man weiß eher wo(nach) man suchen muss“ (Studentenzitat, siehe Tabelle 10).

Einige Studenten schätzen es besonders, in Ruhe, ohne Druck und Angst den virtuellen Patienten zu untersuchen. Andere Studierende vermissen hingegen den Patientenkontakt. Der fehlende Kontakt zum Patienten aber auch zu anderen Kommilitonen (beim Selbststudium) ist ein Nachteil des virtuellen Studiums und des Telelearnings. Der Trend zur Individualisierung des Lernens und die soziale Vereinsamung der Lernenden werden in diesem Zusammenhang befürchtet (Krüger-Brand 1999).

Mittlerweile gibt es verschiedene medizinische Multimediaprodukte (Nitzsche 2002; Heimpel 1998). Bei den meisten Systemen besteht die Interaktion darin, beispielsweise einen Patientenfall durch gezielte virtuelle Untersuchungen und Diagnostik zu lösen (Beispiele: EyeSim ${ }^{\odot}$, go surgery). Diese Multimediaprodukte vermitteln vor allem theoretisches Wissen, das praxisbezogen angewendet werden soll. Sie sind insofern nicht mit dem virtusMED-System vergleichbar, da keine manuelle Fertigkeit geschult wird, wie sie beispielsweise zum Erlernen der Sonographie nötig ist. Betrachtet man die Handhabung der Untersuchungssonde in Kombination mit dem Torso, handelt es sich bei dem virtusMED-System um eine relativ realitätsnahe Simulation (einem sog. Mock-Up). Gerade diese Eigenschaft ermöglicht ein ganzheitliches Lernen (Teistler 2004) und wird von den Studenten sehr geschätzt. Simulationsprogramme, bei denen gezielt eine ganz bestimmte Handhabung bzw. Fertigkeit geübt wird, sind unter den ELearning Methoden weniger häufig vertreten als Präsentationssysteme oder tutorielle Systeme.

Im Bereich der sonographischen Ausbildung finden sich zurzeit nur wenige Simulatoren, die außerdem schwer miteinander vergleichbar sind, da sie auf unterschiedlichen Daten basieren. Im Fachgebiet der Gynäkologie und Geburtshilfe gibt es jedoch mittlerweile einige Ultraschallsimulatoren. Ein Beispiel hierfür ist das SONOFIT Gerät (SONOFIT GmbH), das bereits zu Ausund Fortbildungszwecken genutzt wird. Das virtusMED-System könnte in Zukunft Anwendung in der allgemeinen Sonographieausbildung für Studenten finden und 
wäre damit eine sinnvolle und praktische Ergänzung zum theorielastigen Medizinstudium.

Das Medizinstudium ist trotz neuer Approbationsordnung (Neue AO 2002) immer noch sehr theoretisch aufgebaut. Der Learning-by-doing-Aspekt gerät dabei oft in den Hintergrund. Dass sich Studenten mit Freude selbständig mit neuen Dingen auseinandersetzen und diese ausprobieren, ergaben Beobachtungen während des zweistündigen Ultraschallkurses. Viele Studenten wünschen sich ausdrücklich den vermehrten Einsatz von praxisorientierten Lernprogrammen, die dem virtusMED-System ähneln. Zusätzlich wird der Einsatz des Programms in der anatomischen Ausbildung des vorklinischen Studienabschnitts von einigen Studenten vorgeschlagen. An der Charité in Berlin gibt es seit 1999 das sog. „Trainingszentrum für Ärztliche Fertigkeiten“ (TÄF), das dazu dienen soll, praktische ärztliche Tätigkeiten $\mathrm{zu}$ erlernen und $\mathrm{zu}$ trainieren (http://www.reformstudiengang.charite.de/taef/). An der Universitätsmedizin Göttingen der Georg-August Universität ist ein ähnliches Trainingszentrum (STÄPS - Studentisches Trainingszentrum Ärztlicher Praxis und Simulation) geplant. Die Integration eines Simulationspatienten zum Erlernen der Sonographie bzw. das Einbinden des virtusMED-Systems wäre an dieser Stelle sinnvoll. Auch im Zusammenhang von OSCEs (Oral Standardized Clinical Examination), die mittlerweile immer mehr Einzug in die klinische Ausbildung finden, könnte der Einsatz des virtusMED-Systems realisiert werden.

Alle Funktionen, die das virtusMED-System bereithält, werden von den Studenten als besonders hilfreich eingestuft (siehe Tabelle 8). Das Benennen von Organen hilft den Studenten sich selbständig, ohne Betreuung zurechtzufinden. Es sollte überlegt werden, diese Funktion durch weitere Bezeichnungen anatomischer Strukturen, insbesondere der der Gefäße, zu erweitern.

Trotz der überaus positiven Resonanz auf das virtusMED-System gibt es nach Angaben der Studenten Schwachstellen, die verbesserungswürdig erscheinen. Auf der einen Seite wird die gute und scharfe Bildqualität der 2D-Ansicht (Ultraschallansicht) gelobt, da sie das Lernen besonderes erleichtert. Auf der 
anderen Seite kritisieren viele Studenten den relativ großen Unterschied zum realen Ultraschallbild. Als Lösung könnte ein System mit verschiedenen Schwierigkeitsstufen in betracht gezogen werden. Der erste und einfachste Modus bestünde beispielsweise aus den qualitativ hochwertigen Fotobildern, die durch neue Konfiguration in einem schwereren Modus undeutlicher („schwammiger") würden und mit realen Artefakten versehen werden könnten und dadurch ultraschallnäher wirken würden.

Das virtusMED-System ist ausschließlich in der Lage, Daten des virtuellen Patienten zu generieren. Es handelt sich dabei um Fotodaten, die einen einzigen Moment abbilden, d.h. es ist nicht möglich einen Modus zu erstellen, mit dessen Hilfe beispielsweise zwischen Alter, Geschlecht oder Gesundheitszustand gewechselt werden kann. Einige Studenten bemängeln dies und wünschen sich neben der männlichen auch eine weibliche Darstellung. Die Problematik der Darstellung pathologischer Schnittbilder in Kursen ist bekannt (vgl. Judmaier 2003), aber auch diese Abbildungen sind für Studierende wichtig. Sie würden es befürworten, wenn das virtusMED-System durch pathologische Befunde ergänzt werden könnte. Die Sonographie stellt eine Untersuchungsmethode dar, die sich mit einem sehr dynamischen Bildeindruck befasst (Strauss und Heer 2006). Aus diesem Grund ist der von den Studenten geäußerte Wunsch nach pulsbedingten Gefäßbewegungen verständlich. Die Simulation könnte nach Angaben der Studenten folglich noch realitätsnäher durch das Einspielen von kurzen Filmsequenzen, z.B. der Pulsation von Gefäßen oder der Atemverschieblichkeit von Organen werden.

Aus den studentischen Kommentaren und weiterer Überlegung lassen sich folgende Verbesserungsvorschläge zur Optimierung des virtusMED-Systems ableiten:

1. a) Die aktuelle Version des Computerprogramms sollte durch weitere Funktionen ergänzt werden. Das Auftauchen eines realen Ultraschallbildes (Pop-up Fenster), welches der Position der Untersuchungssonde im jeweiligen Moment entspräche, wäre beispielsweise eine Hilfe. In dieser Form würde der Student einen direkten Vergleich zwischen vereinfachter Darstellung und realem 
Ultraschallbild erhalten. Diese Funktion könnte für häufig verwendete Ultraschallansichten etabliert werden, wie beispielsweise die Leberansicht von sub- und intrakostal, die Nieren in Längs- und Querrichtung, die Milz und andere.

1. b) Der unter 1. a) genannte Verbesserungsvorschlag könnte zusätzlich mit kurzen Filmsequenzen, z.B. dem Pulsieren der großen Gefäße, der Atemverschieblichkeit von Organen oder pathologischen Bildbefunden erweitert werden.

2. Einstellung unterschiedlicher Schwierigkeitsgerade, d.h. der Student kann individuell zwischen leichtem und schwererem (noch realitätsnäherem) Modus wechseln. Im ersten Modus wäre die Bildqualität beispielsweise besonders gut und jede einzelne anatomische Struktur erkennbar. Für den Einstieg und eine erste Orientierung wäre dies ideal. Im nächst höheren Modus wären die Konturen unschärfer und in einem weiteren Modus kämen Bildartefakte hinzu, die diesen Modus ultraschallgleich machen könnten.

3. Eine weitere neue Option „Test“ könnte eine Prüfung simulieren, in der der Student beispielsweise Organe ausmessen oder benennen soll. Eine Auflösung gäbe es im Anschluss. Diese Option lässt dem Student die Möglichkeit seine erworbenen Fertigkeiten selbständig zu überprüfen.

4. Die Funktion „Benennen von Organen“ sollte sich auf noch mehr anatomische Strukturen, insbesondere die Gefäße beziehen, so dass der Student selbständig, ohne Betreuung in der Lage ist, das Computerprogramm zu nutzen.

5. Die Organe, die der 3D-Ansicht hinzufügbar sind, könnten durch die Darstellung der Milz, der Harnblase, der Schilddrüse und weiterer ergänzt werden.

6. Der Styroportorso sollte durch ein gummiartiges, evtl. hautähnlicheres, aber vor allem widerstandsfähigeres und weniger quietschendes Material ersetzt werden.

7. Eine extreme Erweiterung mit Lehrbuchcharakter wäre das Hinzufügen eines Hypertextes, der eine Einführung in die Sonographie und physikalische Grundlagen bieten könnte. Je nach Interesse könnten bestimmte Sachverhalte nachgeschlagen und vertieft werden. 
8. Die graphische Oberfläche wurde von über $90 \%$ der Studenten als gut und sehr gut bewertet, könnte aber, um aktuell zu bleiben, etwas erneuert werden. 


\section{Fazit:}

Die Ergebnisse machen deutlich, dass der Einsatz des virtusMED-Systems besonders zu Beginn der sonographischen Ausbildung sinnvoll erscheint, da es in einfacher Form einen ersten Überblick über dieses Gebiet vermittelt. Die einfache, intuitive Bedienung, die gute Bildqualität und die Benennung der Organe machen es möglich, das Lernprogramm im Selbststudium auch ohne Anleitung und Betreuer zu nutzen. Der virtuelle Patient ist jederzeit verfügbar und untersuchungsbereit. Dies ist ein wesentlicher Vorteil gegenüber dem realen Patienten, da keine Rücksicht auf die Patientenverfügbarkeit genommen werden muss. Als besonders entscheidende Funktion des virtusMED-Systems ist die 3DAnsicht zu beurteilen, da sie dem Studenten ein dreidimensionales Verständnis und eine wesentlich bessere Orientierung bei der gesamten Sonographie vermittelt. Die Simulation ist durch die Handhabung der Untersuchungssonde (Analogie: Ultraschallsonde) und den Einsatz des Torsos besonders praxisnah und interaktiv.

Der große Andrang der Studenten, an dieser Studie teilzunehmen, bzw. an einem zweistündigen Ultraschallkurs, zeigt, dass das Interesse an der Sonographie, aber auch die Bereitschaft, sich in der Freizeit fortzubilden, groß ist. Die Studenten sollten in Zukunft die Möglichkeit erhalten, das virtusMEDSystem jederzeit zu nutzen, da es eine gute und vor allem praxisorientierte Ergänzung im Hinblick auf die bisherige Sonographieausbildung darstellt. Ein Ersatz für eine Ausbildung durch einen erfahrenen Diagnostiker am realen Patienten wird der virtuelle Patient jedoch vermutlich niemals werden. Durch eventuelle Verbesserungen des Lernprogramms könnte der Gewinn an neuen Fertigkeiten und neuem Wissen für die Studenten noch optimiert werden. 


\section{Zusammenfassung}

Einleitung:

Ziel der Arbeit war es herauszufinden, ob bzw. welchen Einfluss das Computerprogramm virtusMED auf das Erlernen der Ultraschalluntersuchung bei Studenten hat und ob es sinnvoll ist, dieses zukünftig in die Lehre zu integrieren, um diese praxisorientierter zu gestalten. Darüber hinaus sollte analysiert werden, wie die Studenten Funktion und Gebrauch des Lernprogramms bewerten, um das virtusMED-System gegebenenfalls zu verbessern.

\section{Material und Methoden:}

Die Bearbeitungszeiten sowie die Messergebnisse zu virtuell und real gestellten Sonographieaufgaben wurden erfasst. Das Gesamtkollektiv umfasste 69 Studenten, die an einem zweistündigen Ultraschallkurs teilnahmen. 34 Studenten (Gruppe A) erhielten ein Vortraining mit dem virtusMED-System und wurden anschließend an die reale Sonographie herangeführt. 35 Student (Gruppe B) durchliefen den Kurs in umgekehrter Reihenfolge. Beide Gruppen wurden mit einem selbst konzipierten Fragebogen über demographische Faktoren sowie ihre Meinung zur Funktion und zum Gebrauch des virtusMEDSystems befragt.

Zur statistischen Auswertung kamen neben dem Mittelwert, der Standardabweichung, dem Median und der Spannweite, der prozentualen Abweichung vom Referenzwert (vom Versuchsleiter ermittelter Mittelwert) sowie Varianzanalysen, t-Tests und Normalverteilungsanalysen zur Anwendung. Das Signifikanzniveau wurde bei $5 \%$ angesetzt.

\section{Ergebnisse:}

Von den 69 Studenten des Gesamtkollektivs waren 54 Frauen $(78,26 \%)$ und 15 Männer (21,74\%). 57 Studenten (82,61\%) stammten aus dem ersten klinischen Semester. Keine sonographischen Erfahrungen hatten 55 der 69 Studenten $(79,71 \%)$, geringe Kenntnisse konnten 14 Studenten (20,29\%) vorweisen, 
welche sie beispielsweise durch Famulatur, Zivildienst oder einen neunzigminütigen universitären Einführungskurs in die Sonographie erworben hatten.

Für die virtuell gelösten Aufgaben erzielten alle Studenten sehr ähnliche Messwerte, was sich in einer geringen Gesamtstandardabweichung widerspiegelt. Im Vergleich dazu ergaben die Messergebnisse bei der realen Sonographie eine deutlich höhere Standardabweichung.

Die Gruppe mit dem virtusMED-Vortraining (Gruppe A) erzielte signifikant kleinere Messergebnisse für die gestellten Aufgaben, als die Gruppe, die sofort an das Ultraschallgerät herangeführt wurde (Gruppe B).

Die Genauigkeit der Messergebnisse steht statistisch gesehen weder mit dem Geschlecht, der Semesteranzahl noch der gering unterschiedlichen Sonographieerfahrung im Zusammenhang. Die Zeit, die die Studenten zum Einstellen des Ultraschallbildes benötigten, scheint ebenfalls keinen Einfluss auf die Messgenauigkeit zu haben.

Die Studenten sind der Meinung, dass das virtusMED-System eine nützliche Ergänzung zur herkömmlichen Ausbildung ist, weil sie sich unter anderem in Ruhe einen ersten Überblick über die Sonographie verschaffen können, eine bessere (dreidimensionale) Orientierung erhalten, das System jederzeit einsetzbar, beliebig oft wiederholbar, interaktiv, praxisorientiert und unkompliziert in der Anwendung ist. Positiv bewerten die Studenten die gute Bildqualität, den fließenden Übergang der Bilder beim Bewegen der Sonde, die realistische Handhabung von Untersuchungssonde und Torso. Besonders hilfreich finden die Studierenden unter anderem die 3D-Ansicht parallel zur 2D-Ansicht (Ultraschallmodus), die Benennung von Organen sowie die Mess- und Freezefunktion.

Ergänzend zu bereits vorhandenen Funktionen des virtusMED-Systems wünschen sich die Studenten pathologische Befunde, reale Bildartefakte, kurze Filmsequenzen mit beispielsweise Pulsation von Gefäßen oder Atemverschieblichkeit von Organen, den Vergleich mit realen Ultraschallbildern oder die Möglichkeit verschiedene Schwierigkeitsstufen einzustellen und einen Prüfungsmodus. Verändern würde ein Großteil der Studenten außerdem das Material des Styroportorsos. 
Die meisten Studenten können sich vorstellen das virtusMED-System auch außerhalb eines Kurses selbständig zu nutzen und wünschen sich den Einsatz dieser und ähnlicher Lernprogramme in der medizinischen Ausbildung.

\section{Kernaussagen}

- Unter identischen Bedingungen (wie bei der virtuellen Sonographie) lassen sich von Studenten annähernd gleiche Messergebnisse erzielen.

- Geschlecht, Semesteranzahl, geringe Sonographievorkenntnisse und Zeit stehen nicht in unmittelbarem Zusammenhang mit der Genauigkeit erzielter Messergebnisse.

- Gruppe A (mit virtusMED-Vortraining) misst die Länge und Breite der Nieren der beiden Testpersonen signifikant kürzer als die Vergleichsgruppe B (ohne virtusMED-Vortraining). Für Höhe und Tiefe der rechten Niere ist kein signifikanter Messunterschied erkennbar.

- Der Einsatz des virtusMED-Systems ist nach Analyse des Fragebogens eine nützliche Ergänzung zur herkömmlichen Ausbildung und wird von den meisten Studenten für die Zukunft gewünscht.

- Die bereits vorhandenen Funktionen des virtusMED-Systems gefallen den Studenten gut und werden als sehr hilfreich eingestuft.

- Verbesserungsbedarf des virtusMED-Systems besteht vor allem im Hinblick auf das Material des Torsos und die 2D-Ansicht (Ergänzung durch reale Ultraschallbilder oder verschiedene Schwierigkeitsstufen). 


\section{Literaturverzeichnis}

Arnold U, Langkafel P (2001): Charité Berlin: Medizinstudium digital?. Dtsch Arztebl $\underline{98},(22), A-1436$

Azuma R T (1997): A Survay of Augmented Reality. Presence: Teleoper. Virtual Environ $\underline{6}$, (4), 355-385

Curie J, Curie P: Développement, par pression, de l'électricité polaire dans les cristaux hémièdres à faces inclines; in: Comptes rendus hebdomadaires des séances de l'Académie des sciences, Band 91, Paris 1880, 294

DEGUM (Deutsche Gesellschaft für Ultraschall in der Medizin) Presseinfo:

Presseinfo September 2004

Presseinfo Mai 2005

Presseinfo Mai 2006

Presseinfo Juli 2006

Presseinfo Juni 2007

online: http://www.degum.de/161.html (zuletzt überprüft am 06.09.2008)

Delorme S, Debus J: Physikalische und technische Grundlagen; in: Sonographie; hrsg. v. Delorme S, Debus J; Georg Thieme Verlag, Stuttgart 2005, 2-24

Docherty C, Hoy D, Topp H, Trinder K (2005): eLearning techniques supporting problem based learning in clinical simulation. Int J Med Inform $\underline{74}$, 527533

Dormeier J, Teistler M (2001): Medizinische Transparenz durch Virtualisierung. Carolo-Wilhelmina (Zeitschrift der TU Braunschweig) 1, 60-67

EyeSim $^{\odot}$ Eye Simulation Page. Simulationen von Augenbewegungen und Pathologien

online: http://cim.ucdavis.edu/eyes/version1/eyesim.htm (zuletzt überprüft am 06.09.2008)

Floto C (2002): Neue Medien in der Medizin: Stellenwert, Chancen und Grenzen. Dtsch Arztbl $\underline{99}$, (27), A-1875-1878

Frey H, Hetzel G, Greis C, Dietrich C F: Ultraschallerzeugung; in: UltraschallKurs Organbezogene Darstellung von Grund-, Aufbau- und Abschlusskurs Nach den Richtlinien von KBV, DEGUM, ÖGUM und SEGUM, hrsg. v. Dietrich C F; Deutscher Ärzte-Verlag, Köln 2006, 2

Frey P (1999): Papier oder PC? Die Neuen Medien auf dem Prüfstand, Eine Umfrage bei 281 Medizinstudierenden. Z Hochschuldidaktik $\underline{3}, 1-5$ 
go surgery. Anamnese- und Diagnostikübungen anhand chirurgischer Patientenfälle

online: http://www.gosurgery.de/login.html (zuletzt überprüft am 06.09.2008)

Heimpel $\mathrm{H}$ : Medizinische Ausbildung und die neuen Informationsmedien; in: Informations- und Wissenstransfer in der Medizin und im Gesundheitswesen, hrsg. Kaltenborn K-F; Vittorio Klostermann Verlag, Frankfurt/M. 1998, 486-505

Hirsch MC: Wissensbasierte Systeme für die medizinische Lehre; in: Informations- und Wissenstransfer in der Medizin und im Gesundheitswesen. hrsg. Kaltenborn K-F; Vittorio Klostermann Verlag, Frankfurt/M. 1999; 401-420.

Hoffmann B (2003): Umfang der Ultraschallausbildung im Amerikanischen Medizinstudium - Eine Datenerhebung, Abstract 15. Varia, 27. Dreiländertreffen ÖGUM - DEGUM - SEGUM 11. - 13.09.2003 in Bregenz

online:

http://www.thieme.de/abstracts/ultraschall/abstracts2003/daten/p8_2.html (zuletzt überprüft am 06.09.2008)

HRET Health Research \& Educational Trust: Do Simulations Reduce the Time to Learn Clinical Skills?

online: http://www.hret.org/hret/programs/simulations.html (zuletzt überprüft am 06.09.2008)

Judmaier G (2003): Ultraschallausbildung - kann die Ausbildung am Phantom die Ausbildungsqualität verbessern?. Ultraschall Med 24, 231-233

Kratochwil A: Physik; in: Ultraschalldiagnostik in der Inneren Medizin, Chirurgie und Urologie; Atlas und Lehrbuch; hrsg. Kratochwil A unter Mitarbeit von Nowotny-Jantsch C; Georg Thieme Verlag, Stuttgart 1977, 1-7

Kratzer W, Pfeiffer M, Adler G (2000): Aus- und Weiterbildung in der AbdomenSonographie an den deutschen Universitäten - Eine Bestandsaufnahme. Internist $\underline{41}$, 37-40

Krüger-Brand H E (1999): Multimedia in der medizinischen Aus- und Fortbildung: Virtuelle Lernszenarios. Dtsch Arztebl $\underline{96},(18), 18$

Krüger-Brand H E (2002): E-Learning in der Medizin: Vor dem Durchbruch. Dtsch Arztebl 99, (22), A-1491-1493

Lernstudio der Chirurgie (Georg-August-Universität Göttingen) online: http://wwwuser.gwdg.de/ chirurg/lernstudio/ (zuletzt überprüft am 06.09.2008) 
Maul H, Scharf A, Baier P, Wüsterman M, Günter H H, Gebauer G, Sohn C (2004): Review: Ultrasound simulators: experience with the SonoTrainer and comparative review of the other training systems. Ultrasound Obstet Gynecol; 24 :581-585

Neue AO (Neue Approbationsordnung für Ärzte) Stand 27. Juni 2002

Nitzsche J (2002): Multimedia in der Medizin: Sinnesbezogen und interaktiv. Dtsch Arztebl 99, (39), A-2534-2535

Page D (2002): Virtual patient never complains. PACSweb - News Update. online:

http://www.dimag.com/pacsweb/newsupdate/showArticle.jhtml?articlelD=4 7901478 (zuletzt überprüft am 06.09.2008)

Pretschner D P (2001): Medizinische Informatik- Virtuelle Medizin auf dem Vormarsch. Carolo-Wilhelmina (Zeitschrift der TU Braunschweig) 1, 14-22

Rhein-Zeitung online, Computer \& Medizin: virtusMED ermöglicht 3D-Reise ins Körperinnere, 27.02.2002

onlline: http://rhein-zeitung.de/on/02/02/27/internet/news1.html?a (zuletzt überprüft am 06.09.2008)

Rosendahl J: Multimediales Lernen in der medizinischen Ausbildung: Möglichkeiten, Nutzung, Grenzen. (Fakultät für Sozial- und Verhaltenswissenschaften) Phil. Diss. Jena 2003

\section{SONOFIT:}

online: http://www.sonofit.de/technishe_einfuerung/simulation.html (zuletzt überprüft am 06.09.2008)

Strauss A, Heer I (2006): Fallstricke eines virtuellen Ultraschallausbildungssystems. Gynäkologe $\underline{39}$, 842-846

Teistler M: Zur räumlichen Exploration tomographischer Bilddaten in virtuellen Szenen für medizinische Ausbildung und Diagnostik. Ing. Diss. Braunschweig 2004

The Visible Human Project, Overview. U.S. National Library of Medicine, Bethesda, MD, U.S., 2003 a

online: http://www.nlm.nih.gov/research/visible/visible_human.html (zuletzt überprüft am 06.09.2008)

The Visible Human Project, Fact Sheet, Overview. U.S. National Library of Medicine, Bethesda, MD, U.S., 2003 b

online: http://www.nlm.nih.gov/pubs/factsheets/visible_human.html (zuletzt überprüft am 06.09.2008) 
Trainingszentrum für Ärztliche Fähigkeiten, Charité Universitätsmedizin Berlin, hompage

online: http://www.reformstudiengang.charite.de/taef/ (zuletzt überprüft am 06.09.2008)

Vogel $H$, Knierer L: Niere; in: Maße in der Sonographie und Computertomographie; hrsg. v. Vogel H unter Mitarbeit v. Baumgärtner EM-, Bölkw K-H., Carter R, Galle B, Heibutzki B, Knierer L, Ludewig U, H-G Rohring, Siemsglüß K; ecomed Verlagsgesellschaft $A G$ Co. KG, Landesberg/Lech 1995, 166-167

Weitzel D: Untersuchungen zur sonographischen Organometrie im Kindesalter. Med. Habil.-Schr. Mainz 1978

Wüstermann M, Scharf A, Maul H, Baier P, Sohn C, (2002): Der Ultraschallsimulator: eine effektive Trainingsmethode zur Steigerung der Untersuchungskompetenz bei der Bestimmung fetaler Nackentransparenz. Geburtshilfe Frauenheilkd $\underline{62}, 1183-1187$ 


\section{Anhang}

\subsection{Studentenfragebogen}

8.1.1 Fragebogen Evaluation virtusMED in der sonographischen Lehre

Name:

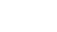

Alter:

Geschlecht: ( ) $\mathrm{m} \quad$ ( ) w

Semesteranzahl:

$\begin{aligned} \text { Erfahrungen am PC: [ ] Textverarbeitung [ ] Internet [ ] Email } & \text { [ ] Programmierung [ ] eigener PC zu Haus }\end{aligned}$

- Frage 1: Haben Sie schon Erfahrungen im Bereich Sonographie?
( ) ja
( ) nein

Wenn ja welche:

- Frage 2: Finden Sie die dreidimensionale Darstellung in der 3DAnsicht zusätzlich zur zweidimensionalen Ansicht der Ultraschallebene hilfreich?

$\begin{array}{llll}\text { auf jeden Fall } & \text { neutral } & \text { gar nicht }\end{array}$
( )
( )
( )
( )
( )

Einschätzung

- Frage 3: Finden Sie den Gebrauch der Sonde und des Styropormodells hilfreich im Hinblick auf die Sonographie? 
- Frage 4: Finden Sie die Messfunktion im Hinblick auf die Sonographie hilfreich?

auf jeden Fall neutral gar nicht keine

Einschätzung
( )
( )
$($ )
( )
( )
( )

- Frage 5: Glauben Sie, dass Sie sich durch die Nutzung des virtusMED-Systems besser bei der Sonographie orientieren können?

$\begin{array}{lll}\text { auf jeden Fall neutral gar nicht } & \text { keine }\end{array}$

Einschätzung
( )
( )
( )
( )
$($ )
( )

- Frage 6: Meinen Sie, der Einsatz des virtusMED-Systems ist eine nützliche Ergänzung zur herkömmlichen Ausbildung?

( ) ja, weil:

( ) nein, weil:

- Frage 7: Können Sie sich vorstellen das virtusMED-System auch außerhalb eines Kurses selbständig zu nutzen?
ja auf jeden ja vielleicht
nein eher nicht nein auf keinen
keine
Fall
Fall
Einschätzung
( )
( )
( )
( )
( )

- Frage 8: Was hat Ihnen am virtusMED-System gut gefallen? Stichpunktartig :

- Frage 9: Was gefällt Ihnen am virtusMED-System nicht? Stichpunktartig : 
8.1.2 Fragen zum Gebrauch des virtusMED-Systems

- Frage 1: Wie gut sind Sie mit der Bedienung des virtusMED-Systems zurechtgekommen?

sehr gut

1

( )

2

( )

3

4

ungenügend

( )

( )

5

6

- Frage 2: Wie gut gefällt Ihnen die grafische Oberfläche des virtusMED-Systems?

\begin{tabular}{cccccc} 
sehr gut & & \multicolumn{3}{r}{ ungenügend } \\
1 & 2 & 3 & 4 & 5 & 6 \\
$($ ) & $($ ) & $($ ) & $($ ) & $($ ) & $($ )
\end{tabular}

- Frage 3: Was würden Sie am virtusMED-System verändern wollen? Stichpunktartig :

- Frage 4: Welche Funktionen am virtusMED-System fanden Sie besonders hilfreich?

Stichpunktartig :

- Frage 6: Was fehlt Ihnen am virtusMED-System im Vergleich zu den herkömmlichen Ausbildungsmöglichkeiten zum Erlernen der sonographischen Untersuchungstechnik?

Stichpunktartig :

Was ich noch sagen wollte: 


\subsection{Komplette Antworten der offenen Fragen zum Fragebogen Evaluation virtusMED in der sonographischen Lehre}

Tabelle 10: Studentische Antworten auf die offenen Fragen zum Fragebogen: „Evaluation virtusMED in der sonographischen Lehre“

\begin{tabular}{|c|c|}
\hline $\begin{array}{l}\text { Frage 6: Meinen Sie, der } \\
\text { Einsatz des virtusMED- } \\
\text { Systems ist eine } \\
\text { nützliche Ergänzung zur } \\
\text { herkömmlichen } \\
\text { Ausbildung? } \\
\text { (von } 69 \text { befragten } \\
\text { Studenten bejahten } \\
\text { diese Antwort } 68 \\
\text { Studenten) }\end{array}$ & 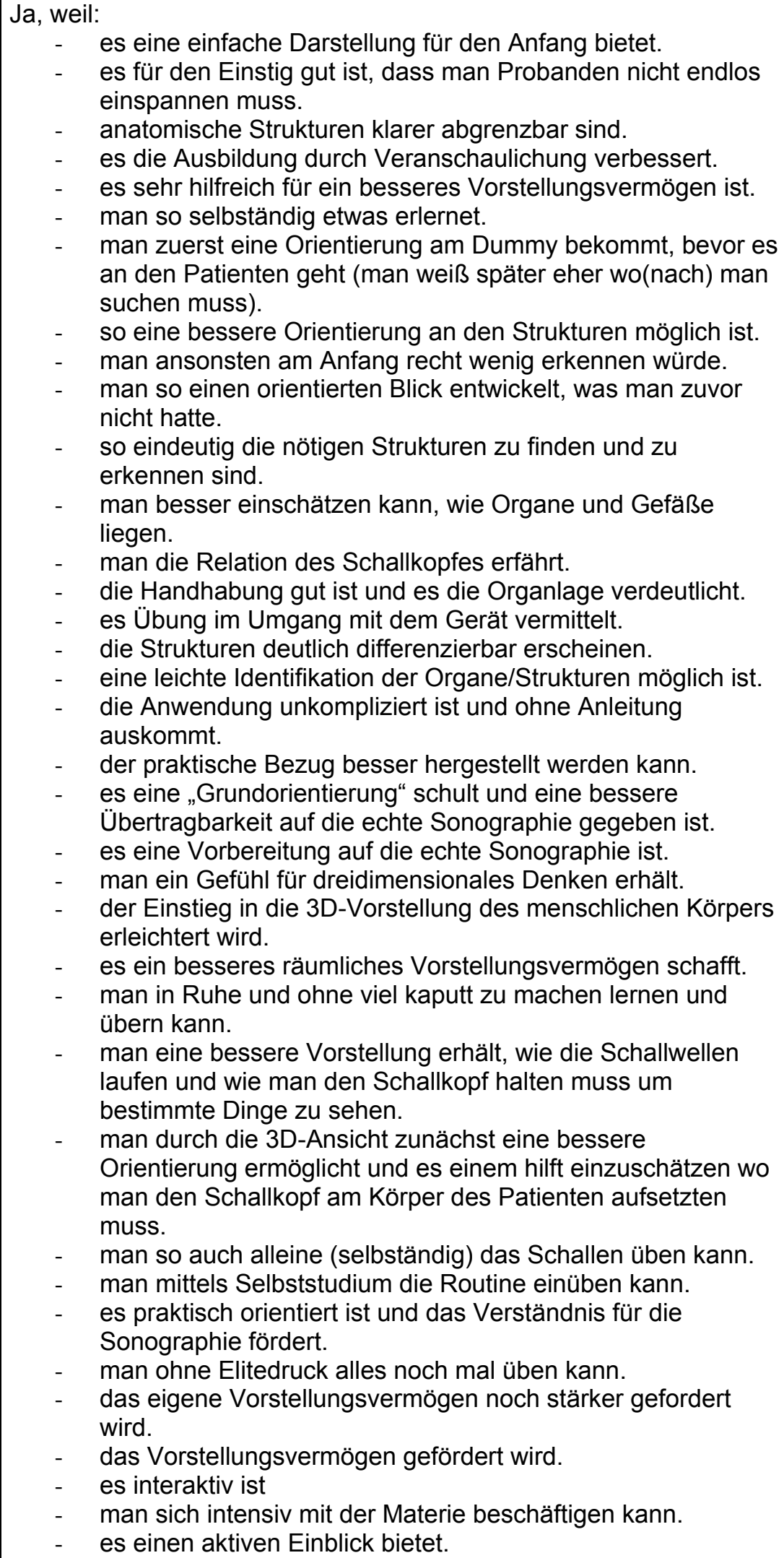 \\
\hline
\end{tabular}




\begin{tabular}{|c|c|}
\hline & $\begin{array}{l}\text { Nein, weil: } \\
\quad-\quad \text { das Bild anders ist. Man sieht mehr als am echten Patienten. }\end{array}$ \\
\hline $\begin{array}{l}\text { Frage 8: Was hat Ihnen } \\
\text { am virtusMED-System } \\
\text { gut gefallen? }\end{array}$ & 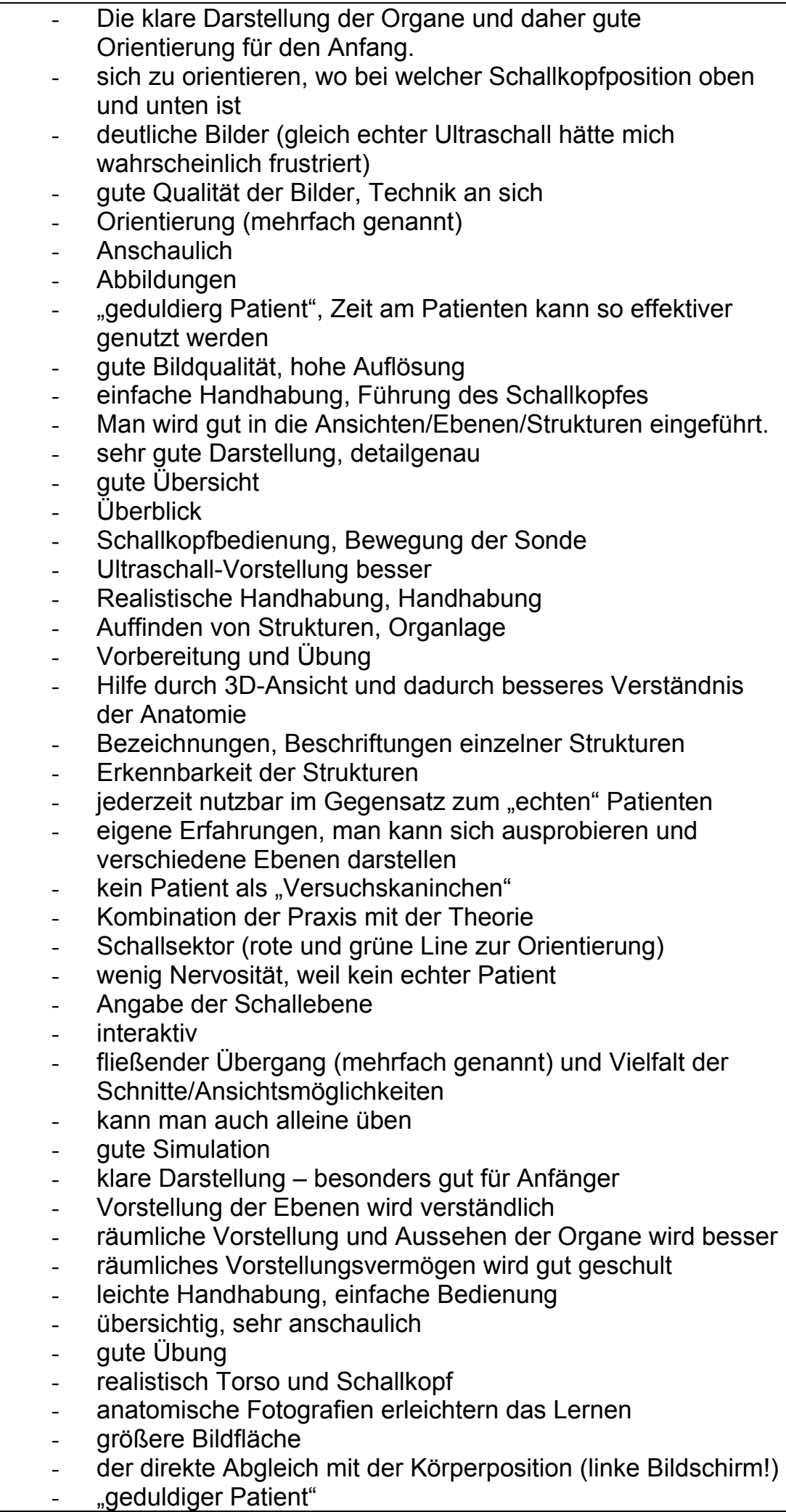 \\
\hline
\end{tabular}




\begin{tabular}{|c|c|}
\hline $\begin{array}{l}\text { Frage 9: Was gefällt } \\
\text { Ihnen am virtusMED- } \\
\text { System nicht? }\end{array}$ & 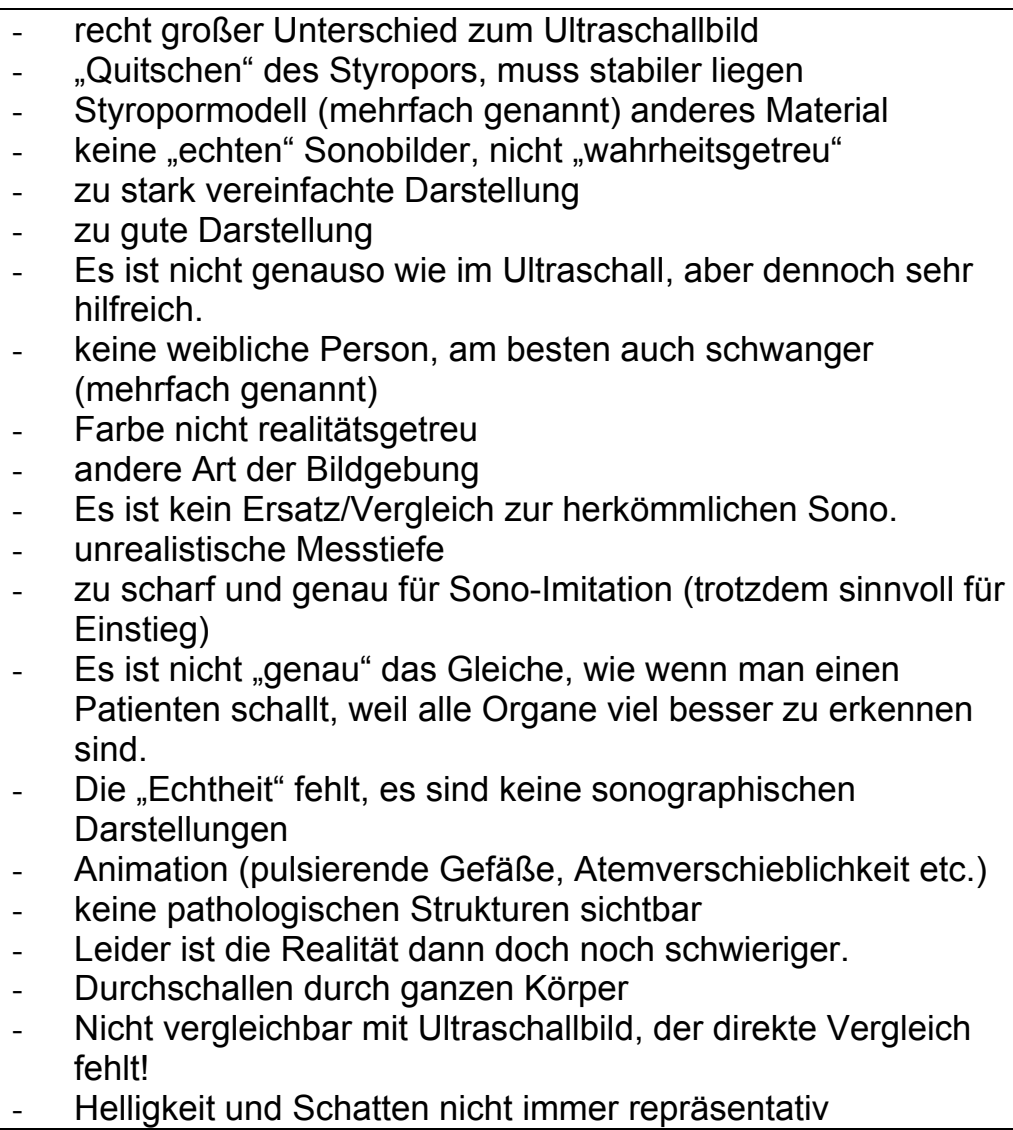 \\
\hline
\end{tabular}

\subsubsection{Komplette Antworten der offenen Fragen zu Fragen zum Gebrauch des virtusMED-Systems}

Tabelle 11: Studentische Antworten auf die offenen Fragen zum Fragebogen: „Fragen zum Gebrauch des virtusMED-Systems"

\begin{tabular}{|c|c|}
\hline $\begin{array}{l}\text { Frage 3: Was würden Sie } \\
\text { am virtusMED-System } \\
\text { verändern wollen? }\end{array}$ & 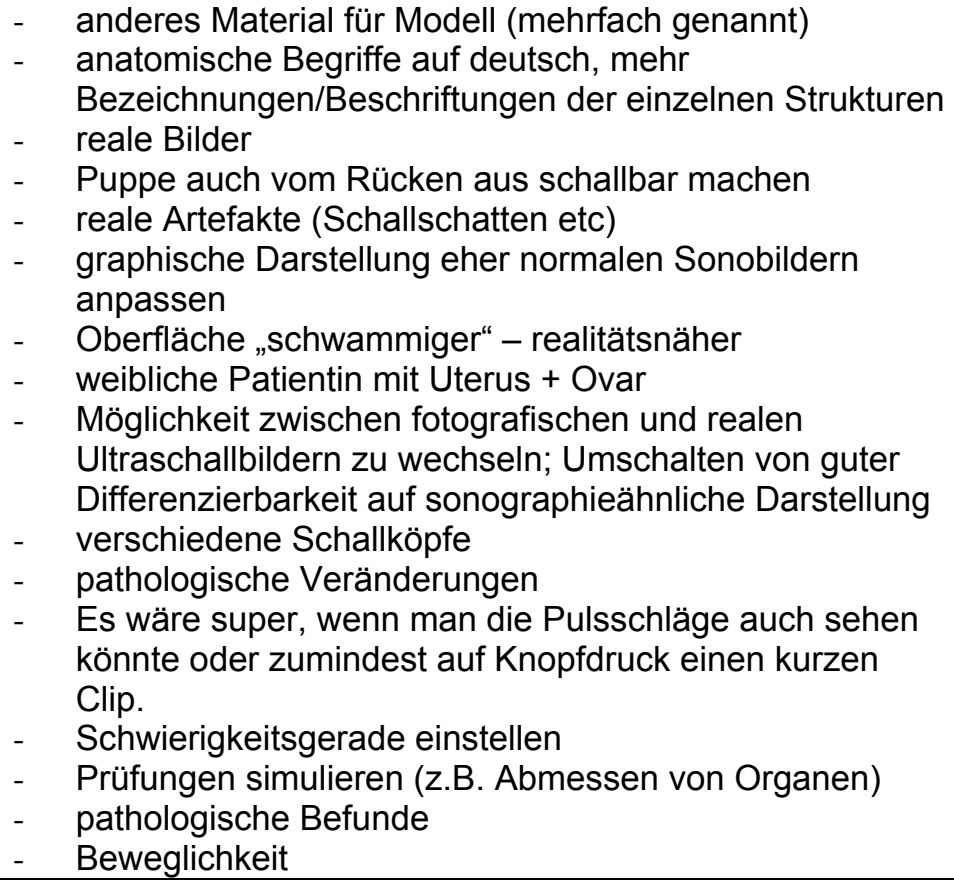 \\
\hline
\end{tabular}




\begin{tabular}{|c|c|}
\hline $\begin{array}{l}\text { Frage 4: Welche Funktion } \\
\text { am virtusMED-System } \\
\text { fanden Sie besonders } \\
\text { hilfreich? }\end{array}$ & $\begin{array}{ll}\text { - } & \text { Benennung der Organe } \\
\text { - } & \text { Schallkopfposition - bessere Orientierungsmöglichkeit, } \\
\text { beide Abbildungen nebeneinander (mehrfach genannt), } \\
\text { man sieht auf welcher Höhe man sich im Körper befindet } \\
\text { - } \quad \text { zusätzliche Ebenendarstellung, dreidimensionale } \\
\text { Orientierungsmöglichkeit an 2. Monitor } \\
\text { - } \quad \text { keine Schattierungen } \\
\text { - } \quad \text { Schallkopfbedienung } \\
\text { - } \quad \text { 3D-Darstellung } \\
\text { - } \quad \text { Schallrichtung lässt sich gut verfolgen / nachvollziehen } \\
\text { - } \quad \text { Größenmessung } \\
\text { - } \quad \text { Patient drehbar } \\
\text { - } \quad \text { Schallrichtung } \\
\text { - } \quad \text { Messfunktion } \\
\text { - } \quad \text { Organe extra einblendbar, zuschalten der Organe auf } \\
\text { dem 3D-Modell } \\
\text { Freeze-Funktion, Funktion mit der man das Bild anhalten } \\
\text { kann } \\
\text { Umgang mit Schallkopf } \\
\text { - Man konnte Organe komplett Durchschallen. Man erhält } \\
\text { eine gute Orientierung der Organlage an der } \\
\text { Körperoberfläche durch das Modell. } \\
\text { rote und grüne Linien zur Orientierung!! } \\
\text { CT-Qualität, auch tiefer liegende Organe erkennbar }\end{array}$ \\
\hline $\begin{array}{l}\text { Frage 6: Was fehlt Ihnen am } \\
\text { virtusMED-System im } \\
\text { Vergleich zu den } \\
\text { herkömmlichen } \\
\text { Ausbildungsmöglichkeiten } \\
\text { zum erlangen der } \\
\text { sonographischen } \\
\text { Untersuchungstechnik? } \\
\text { (diese Frage scheint von } \\
\text { einigen falsch verstanden } \\
\text { worden zu sein) }\end{array}$ & $\begin{array}{cl}\text { - } & \text { Patientenkontakt, Patienteninteraktion fehlt (mehrfach } \\
\text { - } & \text { genannt), mit dem man umgehen muss } \\
\text { - } & \text { reale Bilddarstellung } \\
\text { - } & \text { Atemverschieblichkeit, Pulse, Pulsation von Aorta u. V. } \\
& \text { cava (mehrfach genannt), Schallschatten } \\
\text { - } \quad \text { Sonobilder } \\
\text { - } \quad \text { Einstellung der Frequenzstärke } \\
\text { - } \quad \text { falsche Farbdarstellung } \\
\text { - } \quad \text { Es kann das richtige Schallen nicht ersetzen, da die } \\
\quad \text { Bilder eine zu gute Qualität (keine Schatten etc.) haben. } \\
\text { Falsch verstandene Frage? } \\
\text { - } \quad \text { sehr praxisorientiert } \\
\text { - } \quad \text { sehr genau, alles erkennbar } \\
\text { - } \quad \text { beliebig oft wiederholbar, ohne Patienten „quälen“ zu } \\
\quad \text { müssen }\end{array}$ \\
\hline Was ich noch sagen wollte: & $\begin{array}{ll}\text { - } & \text { gute Erklärung und Hilfestellung im Kurs } \\
\text { - } & \text { Kurs hat mir gut gefallen. Der Kursleiter hat sehr } \\
\text { - } & \text { Vielenschaulich erklärt. } \\
\text { - } & \text { Super Betreuung, vielen Dank. } \\
\text { - } \quad \text { Es ist eine tolle Sache zum besseren Durchblick durch } \\
\text { die Sonographie. } \\
\text { - } \quad \text { sehr hilfreich und sinnvoll } \\
\text { - } \quad \text { Fände das gut, wenn das als Lernobjekt für alle } \\
\text { - Studenten zugänglich wäre! } \\
\text { - War eine tolle Möglichkeit sich der Sonographie zu } \\
\text { nähern und erste Erfahrungen zu sammeln } \\
\text { - Es ist eine sehr gute Möglichkeit den Einstieg in das } \\
\text { komplexe Gebiet zu bekommen. } \\
\text { - Gutes Programm um sich an die Sonographie ran zu } \\
\text { tasten und grobe Übersicht der Organe und ihrer Lage } \\
\text { zu bekommen } \\
\text { Die Betreuung war sehr gut, der Kurs sehr lehrreich und } \\
\text { hat viel Wissen vermittelt. }\end{array}$ \\
\hline
\end{tabular}




\begin{tabular}{|l|ll|}
\hline & - & gut um erste Erfahrungen zu sammeln \\
- & Genial! Muss Einzug in die Ausbildung finden! \\
- & Das virtusMED-System halte ich für eine sehr gute \\
& Möglichkeit um erste Erfahrungen im Umgang mit der \\
& Sonographie zu erlernen. \\
- & Super Kurs! Lohn sich. Sehr hilfreich, die Sachen zu \\
& verstehen Danke! Angenehm in kleiner Gruppe, \\
& besseres Lernen! \\
- & super \\
- & Wäre gut geeignet um eigenständig zu üben (evtl. mit \\
- & HIWl). \\
- & Ein super Einstieg! Der Kursleiter hat damit mein Sono- \\
- & Hoffe es weweckt. \\
& auch für das anatomische Verständnis. \\
- & Hat Spaß gemacht! Hoffe das System wird eingeführt \\
& und wird den Studenten zugänglich gemacht. \\
- & Fände es gut, wenn man zur anatomischen Ausbildung \\
& dieses Verfahren einführen würde. \\
- & Sollte man verwenden im Rahmen eines Sonokurses. \\
- & Das Programm sollte auch für die Vorklinik verwendet \\
& werden. \\
- & Da die Praxis in der Ausbildung viel zu kurz kommt, sollte \\
& es mehr Lernprogramme dieser Art geben. \\
\hline
\end{tabular}

\subsection{Messergebnisse reale Sonographie}

\subsubsection{Gruppe A (erst virtusMED) Testperson und Testperson $_{2}$}

Tabelle 12: Messergebnisse der Gruppe A (erst virtusMED) Testperson ${ }_{1}$ und Testperson zu den drei Aufgaben der realen Sonographie, * Messergebnis wurde nicht gewertet

\begin{tabular}{|c|c|c|c|c|c|c|c|c|c|c|c|}
\hline & \multirow[b]{2}{*}{ 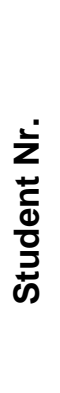 } & \multirow[b]{2}{*}{ 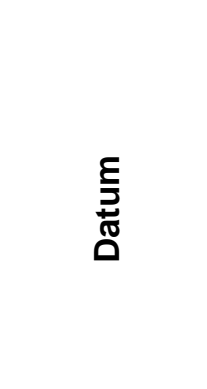 } & \multicolumn{3}{|c|}{ 1. Aufgabe } & \multicolumn{3}{|c|}{ 2. Aufgabe } & \multicolumn{3}{|c|}{ 3.Aufgabe } \\
\hline & & & 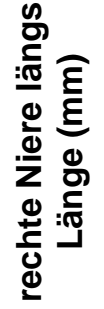 & 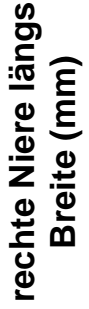 & 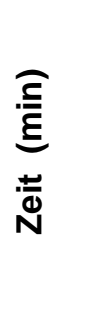 & 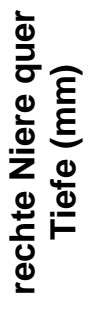 & 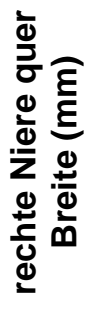 & 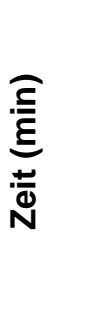 & 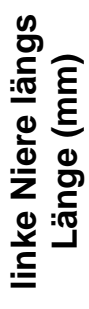 & 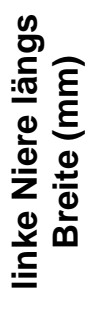 & 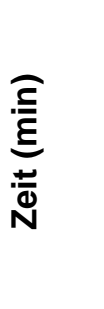 \\
\hline \multirow{14}{*}{ 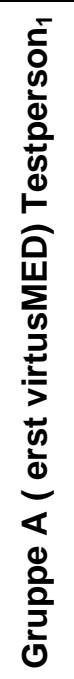 } & 1 & 01.05 .07 & 71,3 & 35,8 & 00:08 & 40,7 & 47,9 & $00: 13$ & * & * & 03:16 \\
\hline & 2 & 01.05 .07 & 78,4 & 37,4 & $00: 17$ & 45,5 & 52,4 & 00:09 & 79,5 & 29,3 & $00: 11$ \\
\hline & 3 & 01.05 .07 & 71,6 & 30,0 & 00:21 & 38,2 & 55,5 & $00: 17$ & 58,0 & 33,1 & 01:42 \\
\hline & 4 & 02.05 .07 & 83,2 & 39,0 & $00: 28$ & 36,8 & 66,6 & $00: 27$ & 71,6 & 43,1 & $00: 49$ \\
\hline & 5 & 02.05 .07 & 85,3 & 37,6 & $00: 18$ & 39,5 & 58,1 & $00: 14$ & 80,9 & 32,9 & $00: 30$ \\
\hline & 6 & 02.05 .07 & 88,0 & 33,8 & $00: 37$ & 38,2 & ${ }^{*}$ & $00: 33$ & $* *$ & 30,8 & $00: 58$ \\
\hline & 7 & 09.05 .07 & 89,0 & 35,6 & $00: 17$ & $*$ & 52,3 & $00: 33$ & 93,0 & 48,2 & $01: 52$ \\
\hline & 8 & 09.05.07 & 73,8 & 27,2 & 00:24 & 37,4 & 58,2 & $00: 18$ & 72,8 & 32,5 & 01:19 \\
\hline & 9 & 09.05.07 & 88,1 & 34,3 & $00: 17$ & 31,7 & 51,5 & $00: 15$ & 71,5 & 35,3 & 00:07 \\
\hline & 10 & $16 . / 22.05 .07$ & 88,6 & 32,4 & $00: 45$ & 38,5 & 59,9 & $00: 35$ & 94,6 & 44,2 & 01:07 \\
\hline & 11 & 16./22.05.07 & 94,6 & 36,3 & $00: 33$ & 42,2 & 43,8 & $00: 11$ & 68,8 & 37,5 & 03:44 \\
\hline & 12 & 30.05 .07 & 81,5 & 33,5 & $00: 25$ & 36,1 & 44,7 & 00:05 & 59,1 & 50,1 & 00:31 \\
\hline & 13 & 30.05 .07 & 102,0 & 35,7 & $00: 35$ & 37,5 & 61,7 & $00: 10$ & 65,6 & 39,3 & 01:34 \\
\hline & 14 & 30.05 .07 & 77,9 & 36,1 & $00: 19$ & 36,4 & 44,7 & $00: 21$ & 91,6 & 40,0 & $00: 37$ \\
\hline
\end{tabular}




\begin{tabular}{|c|c|c|c|c|c|c|c|c|c|c|c|}
\hline & \multicolumn{2}{|c|}{ Mittelwert } & 83,81 & 34,62 & $00: 25$ & 38,36 & 53,64 & $00: 19$ & 75,58 & 38,18 & 01:18 \\
\hline & \multicolumn{2}{|c|}{ Median } & 84,25 & 35,65 & $00: 23$ & 38,20 & 52,40 & $00: 16$ & 72,20 & 37,50 & 01:02 \\
\hline & \multicolumn{2}{|c|}{$\begin{array}{l}\text { Standard- } \\
\text { abweichung }\end{array}$} & 8,8 & 3,1 & $00: 10$ & 3,3 & 7,2 & $00: 10$ & 12,5 & 6,7 & 01:05 \\
\hline & \multicolumn{2}{|c|}{ Minimum } & 71,3 & 27,2 & $00: 08$ & 31,7 & 43,8 & 00:05 & 58,0 & 29,3 & 00:07 \\
\hline & \multicolumn{2}{|c|}{ Maximum } & 102,0 & 39,0 & $00: 45$ & 45,5 & 66,6 & 00:35 & 94,6 & 50,1 & 03:44 \\
\hline \multirow{25}{*}{ 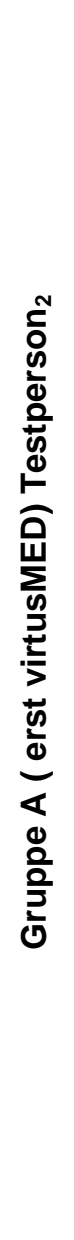 } & 15 & 18.04 .07 & 89,1 & 41,4 & $01: 40$ & 55,4 & 49,4 & $00: 46$ & 81,5 & 45,7 & $00: 45$ \\
\hline & 16 & 18.04 .07 & 100,8 & 43,5 & $02: 57$ & 44,2 & 51,7 & $00: 43$ & 82,3 & 56,9 & $00: 34$ \\
\hline & 17 & 18.04 .07 & 98,6 & 42,2 & $00: 10$ & 45,5 & 41,0 & $00: 40$ & 69,4 & 46,7 & $02: 48$ \\
\hline & 18 & 24.04 .07 & 87,6 & 38,9 & $01: 35$ & 48,3 & 54,4 & $01: 24$ & 91,7 & 42,9 & $02: 15$ \\
\hline & 19 & 24.04 .07 & 89,3 & 39,5 & $00: 30$ & ${ }^{*}$ & 46,1 & $00: 30$ & 87,0 & 36,1 & 01:04 \\
\hline & 20 & 24.04 .07 & 93,4 & 42,0 & $00: 55$ & 39,7 & 55,0 & $00: 17$ & 94,7 & 49,0 & $02: 43$ \\
\hline & 21 & 31.06 .07 & 95,9 & 37,1 & 00:06 & 53,0 & 32,8 & 00:09 & 84,7 & 40,7 & $01: 11$ \\
\hline & 22 & 31.06 .07 & 99,2 & 37,5 & 00:04 & 53,0 & 44,0 & $00: 19$ & 108,0 & 45,4 & $02: 12$ \\
\hline & 23 & 31.06 .07 & 106,0 & 42,6 & 00:06 & 60,6 & 59,1 & $00: 22$ & 97,0 & 42,2 & $01: 12$ \\
\hline & 24 & 05.06 .07 & 88,6 & 38,4 & 00:09 & 46,2 & 48,7 & $00: 13$ & 92,2 & 44,4 & $00: 22$ \\
\hline & 25 & 05.06 .07 & 105,5 & 33,2 & $00: 21$ & 62,4 & 56,4 & $00: 13$ & 97,3 & 54,1 & $00: 17$ \\
\hline & 26 & 05.06 .07 & $*$ & $*$ & $00: 14$ & 32,6 & 55,1 & $00: 18$ & 87,6 & 48,7 & $01: 49$ \\
\hline & 27 & 07.06 .07 & 92,6 & * & $00: 16$ & 42,6 & 68,9 & $00: 18$ & 93,3 & 39,5 & $00: 10$ \\
\hline & 28 & 07.06 .07 & 98,7 & 35,8 & $00: 21$ & 41,5 & 40,8 & $00: 13$ & 95,3 & 45,6 & 00:07 \\
\hline & 29 & 07.06 .07 & 84,4 & 35,8 & 01:35 & 46,7 & 45,8 & 01:07 & 99,9 & 40,6 & $00: 24$ \\
\hline & 30 & 13.06 .07 & 92,3 & 38,5 & $00: 25$ & 36,4 & 50,8 & $00: 23$ & 80,3 & 40,2 & $00: 35$ \\
\hline & 31 & 13.06.07 & 81,3 & 37,5 & $00: 32$ & 65,3 & 49,4 & $00: 42$ & 99,9 & 49,2 & $00: 38$ \\
\hline & 32 & 13.06 .07 & 79,1 & 36,6 & 00:09 & 59,5 & 47,7 & 00:31 & 99,5 & 38,0 & $00: 27$ \\
\hline & 33 & 21.06 .07 & 81,1 & 35,1 & $00: 31$ & 43,3 & 55,4 & $00: 23$ & 93,2 & 58,2 & $00: 58$ \\
\hline & 34 & 21.06 .07 & 99,2 & 36,7 & $00: 23$ & 47,9 & 50,3 & $00: 15$ & 103,5 & 46,8 & $00: 30$ \\
\hline & \multicolumn{2}{|c|}{ Mittelwert } & 92,77 & 38,46 & 00:39 & 48,64 & 50,14 & $00: 29$ & 91,92 & 45,55 & 01:03 \\
\hline & \multicolumn{2}{|c|}{ Median } & 92,60 & 37,95 & 00:22 & 46,70 & 49,85 & $00: 23$ & 93,25 & 45,50 & $00: 42$ \\
\hline & \multicolumn{2}{|c|}{$\begin{array}{l}\text { Standard- } \\
\text { abweichung }\end{array}$} & 8,0 & 2,9 & $00: 45$ & 9,0 & 7,7 & 00:19 & 9,2 & 6,0 & $00: 51$ \\
\hline & \multicolumn{2}{|c|}{ Minimum } & 79,1 & 33,2 & 00:04 & 32,6 & 32,8 & 00:09 & 69,4 & 36,1 & 00:07 \\
\hline & \multicolumn{2}{|c|}{ Maximum } & 106,0 & 43,5 & $02: 57$ & 65,3 & 68,9 & 01:24 & 108,0 & 58,2 & $02: 48$ \\
\hline
\end{tabular}




\subsubsection{Gruppe B (erst Sono) Testperson und Testperson $_{2}$}

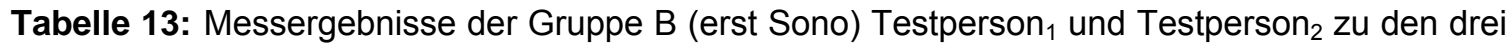
Aufgaben der realen Sonographie, * Messergebnis wurde nicht gewertet

\begin{tabular}{|c|c|c|c|c|c|c|c|c|c|c|c|}
\hline & \multirow[b]{2}{*}{ 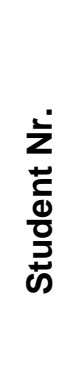 } & \multirow[b]{2}{*}{ 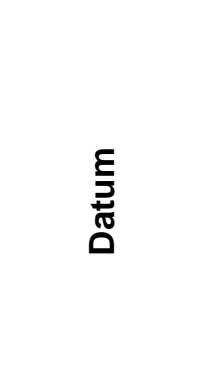 } & \multicolumn{3}{|c|}{ 1. Aufgabe } & \multicolumn{3}{|c|}{ 2. Aufgabe } & \multicolumn{3}{|c|}{ 3. Aufgabe } \\
\hline & & & 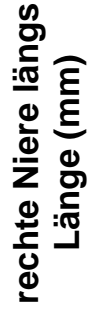 & 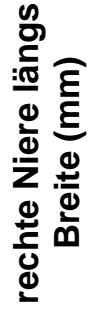 & 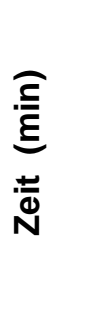 & 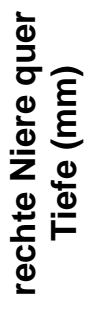 & 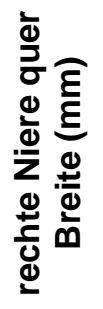 & 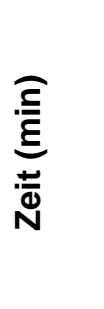 & 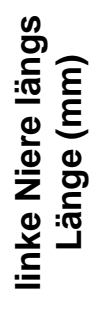 & 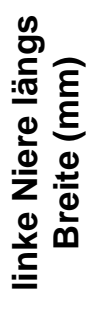 & 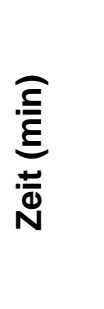 \\
\hline \multirow{23}{*}{ 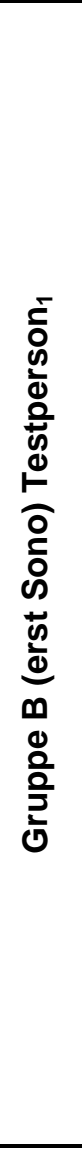 } & 35 & 25.04 .07 & 92,8 & 35,9 & $00: 46$ & 35,9 & 59,5 & $01: 26$ & 104,6 & 56,3 & 03:19 \\
\hline & 36 & 25.04 .07 & ${ }^{*}$ & ${ }^{*}$ & $00: 18$ & 34,4 & 54,4 & $00: 32$ & 95,6 & $* *$ & $01: 35$ \\
\hline & 37 & 25.04 .07 & 84 & 32,8 & $00: 24$ & 37,7 & 73,9 & $00: 33$ & 86,1 & 48,7 & $01: 59$ \\
\hline & 38 & 04.05 .07 & 78,7 & 37,9 & $00: 14$ & 35,3 & 64,3 & 00:09 & 84,4 & 31,7 & $00: 17$ \\
\hline & 39 & 04.0 & * & 37,7 & $00: 12$ & 48 & 43,7 & $00: 21$ & 91,3 & 41,6 & 02:35 \\
\hline & 40 & 04.05 & 83,3 & 43,2 & $00: 09$ & 46,4 & 59,4 & $00: 42$ & * & 38,9 & 03:11 \\
\hline & 41 & 23.05 .07 & 87,3 & 32,6 & $00: 35$ & 39,6 & 69,1 & $01: 32$ & 97,3 & ${ }^{*}$ & $01: 25$ \\
\hline & 42 & 23.05.07 & 80,2 & 28,7 & $00: 36$ & 43,3 & 57,7 & $00: 41$ & 90 & 36,1 & 00:11 \\
\hline & 43 & 23.05.07 & 91,5 & 35,7 & $00: 27$ & 46,1 & 56,3 & $00: 51$ & 96 & 58,7 & $01: 11$ \\
\hline & 44 & 30.05 .07 & 89,8 & 40,4 & $01: 10$ & 39,3 & 54,8 & $00: 42$ & 62 & 40,4 & $01: 15$ \\
\hline & 45 & 30.05 .07 & 83,7 & 34,3 & $00: 22$ & 51,9 & 60,6 & $00: 38$ & 76,5 & 43,6 & 01:54 \\
\hline & 46 & 30.05 .07 & 90,5 & 41,1 & 00:31 & 38,2 & 51,2 & $00: 47$ & 85,1 & 44 & 00:11 \\
\hline & 47 & $04 / 08.06 .07$ & 103 & 38,8 & 01:08 & 38 & 50,3 & $00: 23$ & 82,3 & 52,6 & $00: 31$ \\
\hline & 48 & 04/08.06.07 & 82,2 & 38,2 & $00: 52$ & 41,6 & 44,3 & $00: 50$ & 65,9 & 44,4 & $00: 50$ \\
\hline & 49 & $04 / 08.06 .07$ & 85,1 & 36,1 & $00: 23$ & 35,3 & 66,4 & 00:08 & 77,2 & 46,5 & $00: 16$ \\
\hline & 50 & 11.06 .07 & 92,7 & 32,1 & $00: 12$ & 41,3 & 50,4 & $00: 17$ & 86,7 & 56,1 & 01:14 \\
\hline & 51 & 11.06 .07 & 73,4 & 29,6 & $00: 42$ & 36,4 & * & $00: 44$ & 91,9 & 43,3 & $00: 46$ \\
\hline & 52 & 11.06 .07 & 77,5 & 41,8 & $00: 22$ & 47,3 & 50,1 & $00: 54$ & 92,7 & 52,1 & $00: 25$ \\
\hline & \multicolumn{2}{|c|}{ Mittelwert } & 85,98 & 36,29 & 00:31 & 40,89 & 56,85 & 00:41 & 86,21 & 45,94 & 01:17 \\
\hline & \multicolumn{2}{|c|}{ Median } & 84,55 & 36,10 & $00: 26$ & 39,45 & 56,30 & $00: 42$ & 86,70 & 44,20 & 01:12 \\
\hline & \multicolumn{2}{|c|}{$\begin{array}{l}\text { Standard- } \\
\text { abweichung }\end{array}$} & 7,3 & 4,2 & $00: 18$ & 5,2 & 8,4 & $00: 22$ & 11,1 & 7,7 & $00: 59$ \\
\hline & \multicolumn{2}{|c|}{ Minimum } & 73,4 & 28,7 & 00:09 & 34,4 & 43,7 & 00:08 & 62,0 & 31,7 & 00:11 \\
\hline & \multicolumn{2}{|c|}{ Maximum } & 103,0 & 43,2 & 01:10 & 51,9 & 73,9 & 01:32 & 104,6 & 58,7 & 03:19 \\
\hline \multirow{14}{*}{ 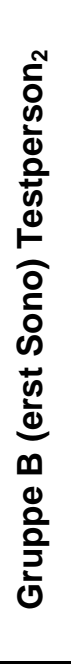 } & 53 & 25.04.07 & 97,8 & 42,1 & $00: 16$ & 51,8 & 52,7 & $00: 21$ & 105,5 & 45 & $00: 17$ \\
\hline & 54 & 25.04 .07 & 96,3 & 38,6 & $00: 39$ & 34,8 & 50,6 & $01: 55$ & 99,6 & 48,7 & $01: 31$ \\
\hline & 55 & & 104,3 & 39,7 & $00: 3$ & * & 51,3 & 00: & 99 & 42,7 & $01: 22$ \\
\hline & 56 & 03.05 .07 & 96,3 & 38 & 01:07 & * & 45,8 & 00:31 & 95,1 & 43,1 & $02: 15$ \\
\hline & 57 & 03.05 .07 & 100,9 & ${ }^{*}$ & $00: 16$ & 58 & 41 & $01: 42$ & 93,1 & 42,3 & $02: 39$ \\
\hline & 58 & 03.05 .07 & 94,2 & 42,4 & $00: 20$ & * & * & $00: 32$ & 95,9 & 45 & 00:31 \\
\hline & 59 & 07.05 .07 & 90 & 39,8 & $00: 46$ & 54,5 & 44,5 & $00: 27$ & 104,6 & 53 & $00: 27$ \\
\hline & 60 & 07.05 .07 & 90,8 & 39,9 & $00: 29$ & 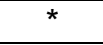 & 58 & $00: 22$ & 93,6 & 46,8 & $00: 24$ \\
\hline & 61 & 14.05 .07 & 88,4 & 46,4 & $00: 24$ & 48,8 & 52,9 & $00: 10$ & 105 & 55,1 & $00: 37$ \\
\hline & 62 & 14.05 .07 & 87,3 & 35,7 & $00: 22$ & 58,3 & 35,6 & $00: 39$ & 79,9 & 47,9 & $00: 26$ \\
\hline & 63 & 14.05 .07 & 104,2 & 42,4 & $00: 37$ & 46,9 & 46 & $00: 38$ & 94,7 & 45,7 & $00: 42$ \\
\hline & 64 & 21.05 .07 & 96,2 & 42,4 & $00: 42$ & 61,2 & 55,6 & $00: 39$ & 98,1 & 42,4 & $01: 53$ \\
\hline & 65 & 21.05 .07 & 96,3 & 38,5 & 01:02 & 44,4 & 48,3 & 00:16 & 99,9 & 52,5 & 00:10 \\
\hline & 66 & 21.05 .07 & 96,7 & 38,4 & 00:09 & 68,8 & 41,7 & $00: 10$ & 98,7 & 42,2 & 01:02 \\
\hline
\end{tabular}




\begin{tabular}{|c|c|c|c|c|c|c|c|c|c|c|}
\hline 67 & 28.06 .07 & 107,5 & 45,7 & $00: 15$ & $*$ & 63 & $02: 24$ & 91,7 & 57,2 & $00: 24$ \\
\hline 68 & 28.06 .07 & 92,5 & 44,1 & $01: 24$ & 58,6 & 41,3 & $00: 31$ & 92,2 & 51,5 & $00: 50$ \\
\hline 69 & 28.06 .07 & 86,5 & 38,8 & $00: 26$ & 48,1 & 44,4 & $00: 24$ & 85 & 37 & $00: 40$ \\
\hline \multicolumn{2}{|l|}{ Mittelwert } & $\mathbf{9 5 , 6 6}$ & $\mathbf{4 0 , 8 1}$ & $\mathbf{0 0 : 3 5}$ & $\mathbf{5 2 , 8 5}$ & $\mathbf{4 8 , 2 9}$ & $\mathbf{0 0 : 4 2}$ & $\mathbf{9 5 , 9 8}$ & $\mathbf{4 6 , 9 5}$ & $\mathbf{0 0 : 5 7}$ \\
\hline Median & $\mathbf{9 6 , 3 0}$ & $\mathbf{3 9 , 8 5}$ & $\mathbf{0 0 : 2 9}$ & $\mathbf{5 3 , 1 5}$ & $\mathbf{4 7 , 1 5}$ & $\mathbf{0 0 : 3 1}$ & $\mathbf{9 5 , 9 0}$ & $\mathbf{4 5 , 7 0}$ & $\mathbf{0 0 : 4 0}$ \\
\hline $\begin{array}{l}\text { Standard- } \\
\text { abweichung }\end{array}$ & $\mathbf{6 , 1}$ & $\mathbf{3 , 0}$ & $\mathbf{0 0 : 2 1}$ & $\mathbf{9 , 0}$ & $\mathbf{7 , 1}$ & $\mathbf{0 0 : 3 9}$ & $\mathbf{6 , 7}$ & $\mathbf{5 , 4}$ & $\mathbf{0 0 : 4 4}$ \\
\hline \multicolumn{2}{|l|}{ Minimum } & $\mathbf{8 6 , 5}$ & $\mathbf{3 5 , 7}$ & $\mathbf{0 0 : 0 9}$ & $\mathbf{3 4 , 8}$ & $\mathbf{3 5 , 6}$ & $\mathbf{0 0 : 1 0}$ & $\mathbf{7 9 , 9}$ & $\mathbf{3 7 , 0}$ & $\mathbf{0 0 : 1 0}$ \\
\hline \multicolumn{2}{|l|}{ Maximum } & $\mathbf{1 0 7 , 5}$ & $\mathbf{4 6 , 4}$ & $\mathbf{0 1 : 2 4}$ & $\mathbf{6 8 , 8}$ & $\mathbf{6 3 , 0}$ & $\mathbf{0 2 : 2 4}$ & $\mathbf{1 0 5 , 5}$ & $\mathbf{5 7 , 2}$ & $\mathbf{0 2 : 3 9}$ \\
\hline
\end{tabular}

\subsubsection{Versuchsleiter (Goldstandard) Testperson T $_{1}$ und Testperson 2}

Tabelle 14: Messergebnisse des Versuchsleiters (Goldstandard) Testperson ${ }_{1}$ und Testperson ${ }_{2}$ zu den drei Aufgaben der realen Sonographie, * Messergebnis wurde nicht gewertet

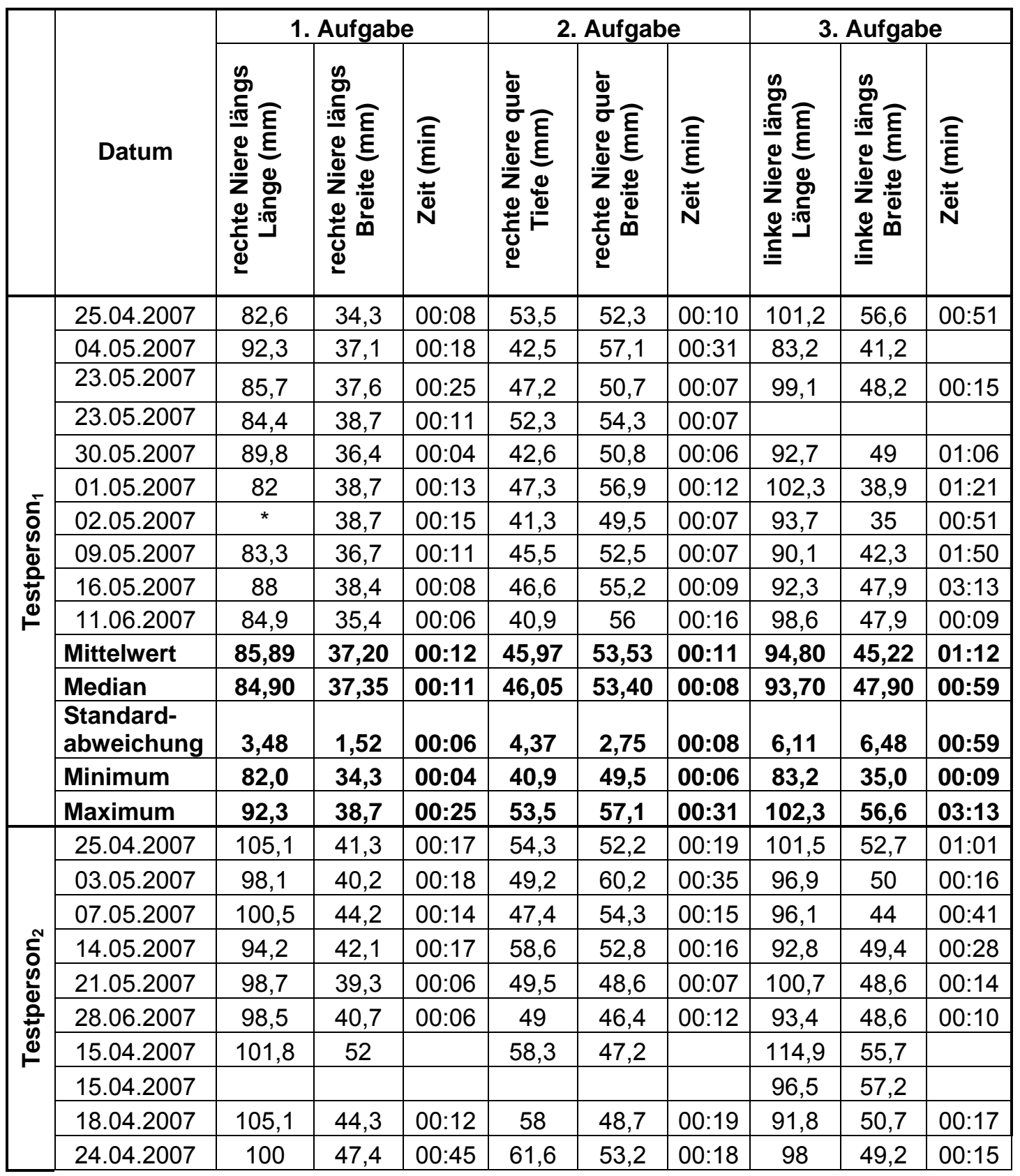




\begin{tabular}{|l|l|c|c|c|c|c|c|c|c|c|}
\hline 31.06 .2007 & 101 & 40,7 & $00: 08$ & 60,9 & 58,4 & $00: 15$ & 95,9 & 46,2 & $00: 12$ \\
\hline 05.06 .2007 & 98 & 41,2 & $00: 06$ & 60,8 & 60 & $00: 06$ & 96,6 & 48,4 & $00: 21$ \\
\hline 07.06 .2007 & 93,9 & 37,8 & $00: 11$ & 48,3 & 44,5 & $00: 14$ & 98,3 & 44,3 & $00: 04$ \\
\hline 13.06 .2007 & 99,1 & 39,9 & $00: 06$ & 50,6 & 56,5 & $00: 14$ & 97,9 & 49,6 & $00: 12$ \\
\hline 21.06 .2007 & 99,1 & 39,2 & $00: 11$ & 50,4 & 55,4 & $00: 10$ & 101 & 44,4 & $00: 17$ \\
\hline Mittelwert & $\mathbf{9 9 , 5 1}$ & $\mathbf{4 2 , 1 6}$ & $\mathbf{0 0 : 1 4}$ & $\mathbf{5 4 , 0 6}$ & $\mathbf{5 2 , 7 4}$ & $\mathbf{0 0 : 1 5}$ & $\mathbf{9 8 , 1 5}$ & $\mathbf{4 9 , 2 7}$ & $\mathbf{0 0 : 2 1}$ \\
\hline Median & $\mathbf{9 9 , 1 0}$ & $\mathbf{4 0 , 9 5}$ & $\mathbf{0 0 : 1 1}$ & $\mathbf{5 2 , 4 5}$ & $\mathbf{5 3 , 0 0}$ & $\mathbf{0 0 : 1 5}$ & $\mathbf{9 6 , 9 0}$ & $\mathbf{4 9 , 2 0}$ & $\mathbf{0 0 : 1 6}$ \\
\hline $\begin{array}{l}\text { Standard- } \\
\text { abweichung }\end{array}$ & $\mathbf{3 , 2 5}$ & $\mathbf{3 , 7 6}$ & $\mathbf{0 0 : 1 0}$ & $\mathbf{5 , 3 8}$ & $\mathbf{5 , 0 9}$ & $\mathbf{0 0 : 0 7}$ & $\mathbf{5 , 4 4}$ & $\mathbf{3 , 8 4}$ & $\mathbf{0 0 : 1 5}$ \\
\hline Minimum & $\mathbf{9 3 , 9 0}$ & $\mathbf{3 7 , 8 0}$ & $\mathbf{0 0 : 0 6}$ & $\mathbf{4 7 , 4 0}$ & $\mathbf{4 4 , 5 0}$ & $\mathbf{0 0 : 0 6}$ & $\mathbf{9 1 , 8 0}$ & $\mathbf{4 4 , 0 0}$ & $\mathbf{0 0 : 0 4}$ \\
\hline Maximum & $\mathbf{1 0 5 , 1 0}$ & $\mathbf{5 2 , 0 0}$ & $\mathbf{0 0 : 4 5}$ & $\mathbf{6 1 , 6 0}$ & $\mathbf{6 0 , 2 0}$ & $\mathbf{0 0 : 3 5}$ & $\mathbf{1 1 4 , 9 0}$ & $\mathbf{5 7 , 2 0}$ & $\mathbf{0 1 : 0 1}$ \\
\hline
\end{tabular}

\subsection{Messergebnisse virtuelle Sonographie}

\subsubsection{Gruppe A (erst virtusMED) Testperson ${ }_{1}$ und Testperson Th $_{2}$ und Gruppe B (erst Sono) Testperson und Testperson $_{2}$}

Tabelle 15: Messergebnisse der Gruppe A (erst virtusMED) Testperson ${ }_{1}$ und Testperson ${ }_{2}$ und der Gruppe B (erst Sono) Testperson ${ }_{1}$ und Testperson 2 zu den zwei Aufgaben der virtuellen Sonographie, ${ }^{*}$ Messergebnis wurde nicht gewertet

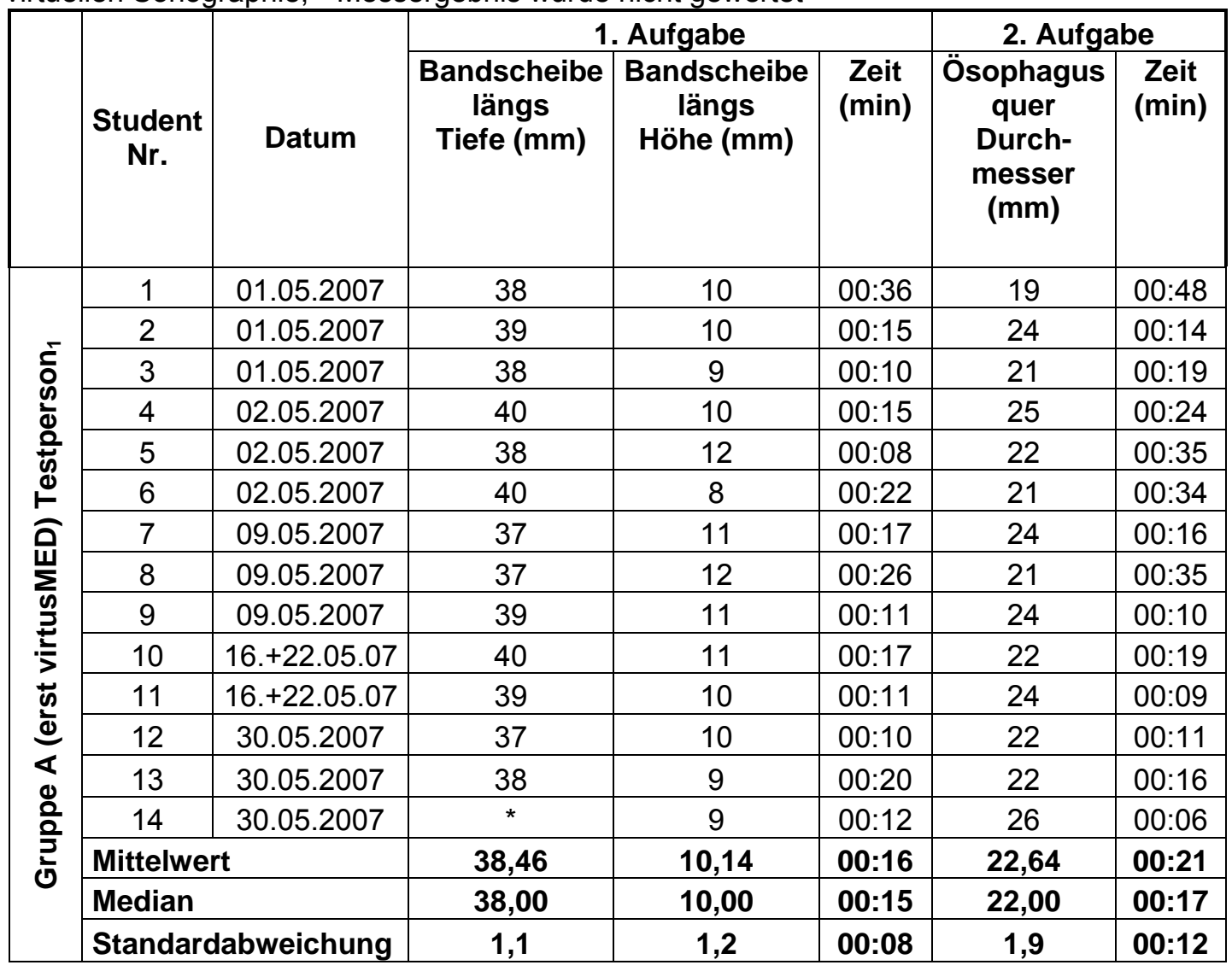




\begin{tabular}{|c|c|c|c|c|c|c|c|}
\hline \multirow{23}{*}{ 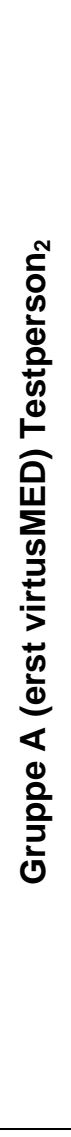 } & 15 & 18.04 .2007 & 41 & 12 & 02:07 & 29 & $02: 35$ \\
\hline & 16 & 18.04 .2007 & 33 & 9 & 01:45 & 29 & $01: 45$ \\
\hline & 17 & 18.04 .2007 & 34 & 11 & $00: 42$ & 25 & $00: 35$ \\
\hline & 18 & 24.04 .2007 & 40 & 10 & $00: 18$ & 22 & $00: 38$ \\
\hline & 19 & 24.04 .2007 & 43 & 11 & $00: 21$ & 21 & $01: 52$ \\
\hline & 20 & 24.04 .2007 & 40 & 10 & 00:09 & 18 & $01: 24$ \\
\hline & 21 & 31.06 .2007 & 39 & 11 & $00: 13$ & 23 & $00: 14$ \\
\hline & 22 & 31.06 .2007 & 39 & 11 & $00: 37$ & 23 & $00: 24$ \\
\hline & 23 & 31.06 .2007 & 39 & 11 & 00:09 & 22 & $00: 58$ \\
\hline & 24 & 05.06 .2007 & 41 & 11 & $00: 12$ & 23 & $00: 23$ \\
\hline & 25 & 05.06 .2007 & 41 & 11 & $00: 15$ & 24 & $00: 22$ \\
\hline & 26 & 05.06 .2007 & 39 & 10 & 00:09 & 24 & 00:08 \\
\hline & 27 & 07.06 .2007 & 41 & 11 & $00: 22$ & 23 & $00: 49$ \\
\hline & 28 & 07.06 .2007 & 40 & 10 & 00:07 & 24 & $00: 24$ \\
\hline & 29 & 07.06 .2007 & 38 & 10 & $00: 20$ & 23 & $00: 24$ \\
\hline & 30 & 13.06.2007 & 38 & 10 & 00:10 & 22 & 00:09 \\
\hline & 31 & 13.06 .2007 & 41 & 10 & $00: 12$ & 21 & $00: 12$ \\
\hline & 32 & 13.06.2007 & 39 & 12 & 01:27 & 25 & $00: 45$ \\
\hline & 33 & 21.06 .2007 & 40 & 9 & 00:19 & 22 & $00: 23$ \\
\hline & 34 & 21.06 .2007 & 39 & 10 & $00: 23$ & 20 & $00: 13$ \\
\hline & \multicolumn{2}{|c|}{ Mittelwert } & 39,25 & 10,50 & 00:31 & 23,15 & $00: 44$ \\
\hline & \multicolumn{2}{|c|}{ Median } & 39,50 & 10,50 & 00:19 & 23,00 & $00: 24$ \\
\hline & \multicolumn{2}{|c|}{ Standardabweichung } & 2,3 & 0,8 & $00: 34$ & 2,6 & $00: 40$ \\
\hline \multirow{21}{*}{ 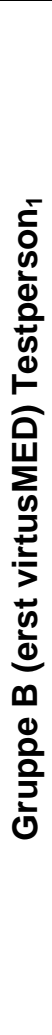 } & 35 & 25.04 .2007 & 38 & 10 & $00: 28$ & 19 & 01:05 \\
\hline & 36 & 25.04 .2007 & 40 & 11 & $00: 21$ & 20 & $00: 00$ \\
\hline & 37 & 25.04 .2007 & 41 & 11 & $00: 11$ & 24 & $01: 00$ \\
\hline & 38 & 04.05 .2007 & 40 & 10 & 00:11 & 27 & $00: 00$ \\
\hline & 39 & 04.05 .2007 & 40 & 12 & $00: 23$ & 25 & $00: 10$ \\
\hline & 40 & 04.05 .2007 & 40 & 11 & 00:12 & 24 & 00:06 \\
\hline & 41 & 23.05 .2007 & 40 & 10 & $00: 26$ & 24 & $00: 11$ \\
\hline & 42 & 23.05 .2007 & 38 & 11 & 00:18 & 24 & 01:05 \\
\hline & 43 & 23.05 .2007 & 40 & 12 & 00:06 & 24 & $00: 29$ \\
\hline & 44 & 30.05 .2007 & 41 & 11 & $00: 58$ & 22 & $00: 16$ \\
\hline & 45 & 30.05 .2007 & 41 & 10 & $00: 28$ & 25 & $00: 20$ \\
\hline & 46 & 30.05 .2007 & 37 & 10 & 00:16 & 24 & $00: 22$ \\
\hline & 47 & $04 / 08.06 .07$ & 39 & 11 & $00: 43$ & 24 & $00: 25$ \\
\hline & 48 & $04 / 08.06 .07$ & $*$ & 9 & 01:14 & 21 & $00: 22$ \\
\hline & 49 & 04/08.06.07 & 40 & 10 & $00: 12$ & 22 & $00: 12$ \\
\hline & 50 & 11.06 .2007 & 39 & 11 & 00:06 & 23 & $00: 17$ \\
\hline & 51 & 11.06.2007 & 36 & 9 & 00:06 & 23 & $00: 17$ \\
\hline & 52 & 11.06 .2007 & 40 & 11 & $00: 13$ & 23 & $00: 29$ \\
\hline & \multicolumn{2}{|c|}{ Mittelwert } & 39,41 & 10,56 & $00: 23$ & 23,22 & $00: 26$ \\
\hline & \multicolumn{2}{|c|}{ Median } & 40,00 & 11,00 & $00: 17$ & 24,00 & 00:21 \\
\hline & \multicolumn{2}{|c|}{ Standardabweichung } & 1,4 & 0,9 & 00:19 & 1,9 & 00:19 \\
\hline
\end{tabular}




\begin{tabular}{|c|c|c|c|c|c|c|c|}
\hline \multirow{20}{*}{ 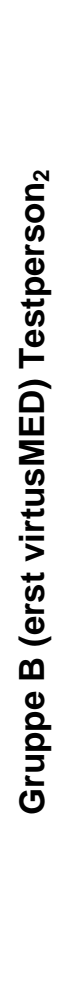 } & 53 & 25.04 .2007 & 41 & 11 & $00: 17$ & 21 & $00: 21$ \\
\hline & 54 & 25.04 .2007 & 40 & 10 & $00: 22$ & 23 & $00: 33$ \\
\hline & 55 & 25.04 .2007 & 40 & 12 & $00: 14$ & 24 & $00: 33$ \\
\hline & 56 & 03.05 .2007 & 38 & 8 & $01: 36$ & 23 & $00: 38$ \\
\hline & 57 & 03.05 .2007 & 40 & 12 & $01: 54$ & 23 & $01: 13$ \\
\hline & 58 & 03.05 .2007 & 35 & 10 & $01: 22$ & * & $00: 28$ \\
\hline & 59 & 07.05 .2007 & 39 & 11 & $00: 10$ & 26 & $00: 14$ \\
\hline & 60 & 07.05 .2007 & 41 & 12 & $00: 36$ & 23 & $00: 21$ \\
\hline & 61 & 14.05 .2007 & 40 & 11 & $00: 21$ & 26 & $00: 14$ \\
\hline & 62 & 14.05 .2007 & 39 & 10 & $00: 51$ & 26 & $00: 34$ \\
\hline & 63 & 14.05 .2007 & 38 & 10 & $00: 22$ & * & $00: 48$ \\
\hline & 64 & 21.05 .2007 & 40 & 11 & $00: 30$ & 23 & $00: 10$ \\
\hline & 65 & 21.05 .2007 & 41 & 10 & $00: 12$ & 21 & $00: 15$ \\
\hline & 66 & 21.05 .2007 & 42 & 11 & $00: 20$ & 27 & $00: 32$ \\
\hline & 67 & 28.06.2007 & 39 & 12 & 00:05 & 27 & 00:09 \\
\hline & 68 & 28.06 .2007 & 43 & 10 & $00: 19$ & 25 & $01: 14$ \\
\hline & 69 & 28.06 .2007 & 38 & 10 & 00:09 & 22 & $00: 23$ \\
\hline & \multicolumn{2}{|c|}{ Mittelwert } & 39,65 & 10,65 & $00: 34$ & 24,00 & $00: 31$ \\
\hline & \multicolumn{2}{|c|}{ Median } & 40,00 & 11,00 & 00:21 & 23,00 & $00: 28$ \\
\hline & \multicolumn{2}{|c|}{ Standardabweichung } & 1,8 & 1,1 & $00: 33$ & 2,0 & $00: 19$ \\
\hline & \multirow{2}{*}{\multicolumn{2}{|c|}{$\begin{array}{l}\text { Gesamtmittelwert } \\
\text { Gesamtmedian }\end{array}$}} & 39,24 & 10,48 & & 23,25 & \\
\hline & & & 40,00 & 10,00 & & 23,00 & \\
\hline & \multicolumn{2}{|c|}{ Gesamt } & 1,80 & 0,96 & & 2,16 & \\
\hline
\end{tabular}

\subsubsection{Versuchsleiter / Referenzwert}

Tabelle 16: Messergebnisse des Versuchsleiters zu den zwei Aufgaben der virtuellen Sonographie

\begin{tabular}{|c|c|c|c|}
\hline & \multicolumn{2}{|c|}{ 1. Aufaabe } & \multirow[b]{2}{*}{\begin{tabular}{|} 
2. Aufgabe \\
Ösophagus \\
quer \\
Durch- \\
messer \\
(mm)
\end{tabular}} \\
\hline & $\begin{array}{c}\text { Bandscheibe } \\
\text { längs } \\
\text { Tiefe }(\mathrm{mm})\end{array}$ & $\begin{array}{l}\text { Bandscheibe } \\
\text { längs } \\
\text { Höhe (mm) }\end{array}$ & \\
\hline \multirow{10}{*}{ Versuchsleiter } & 39 & 11 & 23 \\
\hline & 40 & 11 & 23 \\
\hline & 39 & 11 & 23 \\
\hline & 39 & 10 & 22 \\
\hline & 40 & 11 & 23 \\
\hline & 40 & 10 & 23 \\
\hline & 38 & 11 & 24 \\
\hline & 39 & 12 & 22 \\
\hline & 40 & 11 & 24 \\
\hline & 40 & 11 & 23 \\
\hline Mittelwert & 39,40 & 10,90 & 23,00 \\
\hline Median & 39,50 & 11,00 & 23,00 \\
\hline Standardabweichung & 0,70 & 0,57 & 0,67 \\
\hline
\end{tabular}




\section{Danksagung}

Herzlich danken möchte ich den zahlreichen Studenten, die mit Freude und Engagement an dieser Studie teilnahmen, sowie allen, die mich bei der Erstellung dieser Arbeit unterstützt haben. Mein besonderer Dank gilt:

- meinem Doktorvater, Herrn Prof. Dr. med. Klaus Dresing für die Überlassung des Dissertationsthemas und die freundliche Betreuung, sowie Unterstützung und Korrektur dieser Arbeit.

- Herrn Dr. Oliver Bott für die gute Zusammenarbeit an der Technischen Universität Carolo-Wilhelmina zu Braunschweig

- Katharina Friz und Luise Proske, die ihre private Zeit opferten, um sich geduldig von zahlreichen Studenten sonographisch untersuchen zu lassen.

- Dr. Karthinathan Thangavelu, der mich bei der Fertigstellung der Auswertung mit seinem statistischen Wissen tatkräftig unterstützte und meine Arbeit durch Fragen und Ideen vorantrieb. 


\section{Lebenslauf}

Am 27. April 1983 wurde ich als Tochter des Betriebswirtes Ralf Reihs und der Realschullehrerin Marlies Dittmer in Hamburg geboren.

Meine Grundschulzeit verbrachte ich von 1989 bis 1993 an der Grundschule Tangstedt. Anschließend besuchte ich das Gymnasium Heidberg in Hamburg, Langenhorn, wo ich 2002 die allgemeine Hochschulreife erwarb. Von 1999 bis 2003 erlangte ich parallel zum Abitur die Qualifikation zur staatlich geprüften Chemisch-Technischen Assistentin.

Jeweils einen Monat meines vorklinischen Krankenpflegepraktikums absolvierte ich im Albertinen-Krankenhaus in Hamburg (26.06. - 02.08.2002) sowie im Medizinischen Zentrum Itzehoe (03.02. - 02.03.2003).

Im April 2003 nahm ich an der Georg-August-Universität Göttingen mein Medizinstudium auf. Die ärztliche Vorprüfung bestand ich nach vier vorklinischen Semestern im März 2005. Im Juli 2006 begann ich vorliegende Dissertation.

Für meine jeweils einmonatigen Famulaturen wählte ich die Fächer Pädiatrie (Kinderarztpraxis Herr Dr. med. Warmbrunn, Freiburg), Allgemein- und Unfallchirurgie (Paracelsus Klinik, Henstedt-Ulzburg), Plastische-, Hand- und rekonstruktive Mikrochirurgie (Berufsgenossenschaftliche Unfallklinik Murnau) sowie Plastische und Verbrennungschirurgie (University Clinical Centre, Ljubljana, Slowenien). Eine zweiwöchige Ultraschall-Famulatur absolvierte ich in der Allgemeinchirurgie des Universitätsklinikums Göttingen.

Im August 2008 habe ich das erste Tertial meines Praktischen Jahres für Innere Medizin am Albert-Schweitzer Krankenhaus in Northeim begonnen. Mein Wahlfach Orthopädie werde ich ab Dezember 2008 am Kantonsspital Luzern, Schweiz ableisten. Acht Wochen des dritten Tertails werde ich an der University of Newcastle upon Tyne, England in der Plastischen und Wiederherstellungschirurgie verbringen. Anschließend werde ich die letzten Wochen des chirurgischen Tertials erneut in Northeim absolvieren. 